\title{
TOPOLOGY, DEFECTS, AND TOPOLOGICAL DEFECTS
}

\author{
MARIIA A. LIZUNOVA
}




\title{
Topology, defects, and topological defects
}

\author{
M. A. Lizunova
}


$\mathrm{PhD}$ thesis, Utrecht University, September 2020

ISBN: 978-94-6380-930-6

DOI: https://doi.org/10.33540/82

Cover design: Mariia Lizunova

Cover illustration: Alexander Barnaveli

Printed by: ProefschriftMaken.nl 


\title{
Topology, defects, and topological defects
}

\author{
Topologie, defecten en topologische \\ defecten
}

(met een samenvatting in het Nederlands)

\section{Proefschrift}

ter verkrijging van de graad van doctor aan de Universiteit Utrecht op gezag van de rector magnificus, prof. dr. H.R.B.M. Kummeling, ingevolge het besluit van het college voor promoties in het openbaar te verdedigen op woensdag 16 september 2020 des middags te 12.45 uur

door

\section{Mariia Andreyevna Lizunova}

geboren op 19 juli 1993 te Elektrostal, Rusland 
Promotor: Prof. Dr. C. de Morais Smith

Copromotor: Dr. J. van Wezel 


\section{Contents}

Preface $\quad$ v

List of publications vii

1 Introduction $\quad 1$

2 Introduction to topological transport 5

2.1 Introduction . . . . . . . . . . . . . . . . 5

2.2 Integer quantum Hall effect . . . . . . . . . . . . . . . . . . . . 6

2.3 Dynamics in Laughlin's IQHE cylinder . . . . . . . . . . . . . . . . . 10

3 Visualizing topological transport in charge-ordered materials 13

3.1 Introduction . . . . . . . . . . . . . . . . . . . . 13

3.2 Charge-density waves . . . . . . . . . . . . . . . . . . 14

3.3 Mapping between IQHE and CDW . . . . . . . . . . . . . . . . 15

3.4 Adiabatic transport . . . . . . . . . . . . . . . . . . 17

3.5 Emergence of a mobility edge . . . . . . . . . . . . . . . 21

3.6 Nonadiabatic transport . . . . . . . . . . . . . . . . . 22

3.7 Possible experimental implementation . . . . . . . . . . . . . . 25

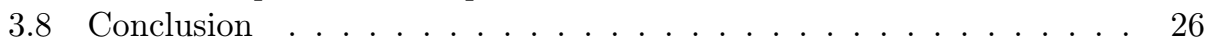

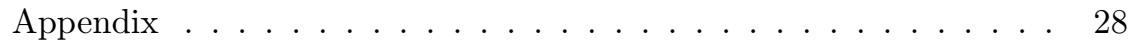

3.A The mean-field Hamiltonian . . . . . . . . . . . . . . . . . 28

3.B The Fourier-transformed Hamiltonian . . . . . . . . . . . . . . . . . 29

3.C The Hamiltonian in matrix form . . . . . . . . . . . . . . . . 31

3.D Calculating the topological invariant . . . . . . . . . . . . . 32

4 Introduction to solitary waves $\quad 35$ 


\section{Contents}

4.1 Introduction . . . . . . . . . . . . . . . . . . 35

4.2 Topological sectors . . . . . . . . . . . . . . . . . . . 36

4.3 Solitons in the sine-Gordon model . . . . . . . . . . . . . . . . . . 38

4.4 Josephson effect . . . . . . . . . . . . . . . . . . . . . . . . . . . . 40

4.5 Kinks in the $\varphi^{4}$ model . . . . . . . . . . . . . . . . . . . . . . . . . . . . .

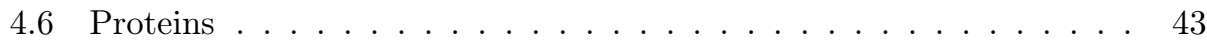

Appendix ............................... 47

4.A Internal kink excitation mode . . . . . . . . . . . . . . . . . 47

4.B Elliptic sine solution . . . . . . . . . . . . . . . . 49

5 Solitary wave propagation in media with step-like inhomogeneities

5.1 Introduction . . . . . . . . . . . . . . . . . 53

5.2 Step-like inhomogeneity in scalar fields . . . . . . . . . . . . . . 54

5.3 Quench in space . . . . . . . . . . . . . . 56

5.3.1 Moving solitary waves: $\zeta_{1}=1$ and $0<\zeta_{2}<\infty \ldots . . . . .56$

5.3.2 Moving solitary waves: $\zeta_{1}=1$ and $0<\zeta_{2}<1 \ldots$. . . . . . 61

5.3.3 Moving solitary waves: $\zeta_{1}=1$ and $\zeta_{2}=0$. . . . . . . . . 62

5.3.4 Static solitary waves on border . . . . . . . . . . . 63

5.3.5 Mirror reflection ................ 66

5.4 Quench dynamics . . . . . . . . . . . . . . . . . . . . 67

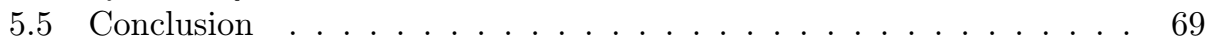

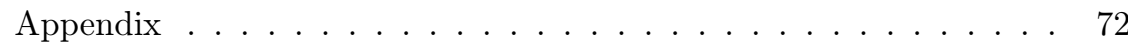

5.A Numerical method . . . . . . . . . . . . . . . . . . 72

5.B Systematic error for $v_{f} \ldots \ldots \ldots \ldots$. . . . . . . . . . 73

6 Kinks and realistic impurity models in the $\varphi^{4}$ theory $\quad 75$

6.1 Introduction . . . . . . . . . . . . . . . . . . 75

6.2 Impurity . . . . . . . . . . . . . . . . . . . . 76

6.3 Kink-impurity interactions . . . . . . . . . . . . . . . 78

6.3.1 Dirac delta limit of the Gaussian profile . . . . . . . . . . . . 78

6.3.2 Impurity strength and width . . . . . . . . . . . . . . 80

6.3.3 The impurity mode................. 81

6.3.4 Lorentzian profile. . . . . . . . . . . . . . . . . 83

6.4 Kink-antikink interactions . . . . . . . . . . . . . . . . 85

6.5 Kink-impurity-antikink interactions . . . . . . . . . . . . . 87

6.5.1 Dirac delta limit of the Gaussian profile . . . . . . . . . . 87

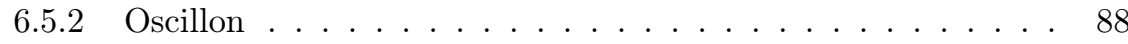

6.5.3 The critical velocity . . . . . . . . . . . . . . . . 90

6.5.4 The impurity mode . . . . . . . . . . . . . . . . 92

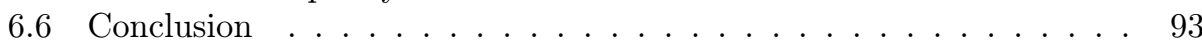


Contents

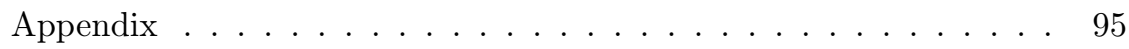

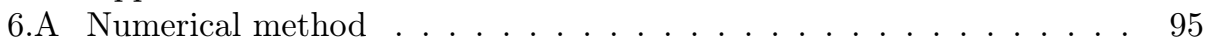

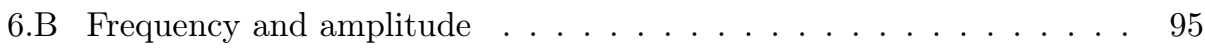

6.C Collective coordinate approximation . . . . . . . . . . . . . . . . 96

6.D Kink-elliptic sine-antikink ansatz . . . . . . . . . . . . . . . 99

$\begin{array}{ll}\text { Bibliography } & 101\end{array}$

$\begin{array}{ll}\text { Summary } & 113\end{array}$

$\begin{array}{ll}\text { Samenvatting } & 115\end{array}$

$\begin{array}{lr}\text { Curriculum Vitae } & 117\end{array}$

$\begin{array}{lr}\text { Acknowledgement } & 119\end{array}$ 



\section{Preface}

This Thesis is the result of four years of work, done at the Institute for Theoretical Physics of the Utrecht University under the supervision of Prof. Dr. Cristiane de Morais Smith and Institute for Theoretical Physics of the University of Amsterdam under the supervision of Dr. Jasper van Wezel. This work is part of the Delta Institute for Theoretical Physics (DITP) consortium, a program of the Netherlands Organization for Scientific Research (NWO) that is funded by the Dutch Ministry of Education, Culture, and Science (OCW).

The whole Thesis is united by the idea of topology and its applications. Nevertheless, we tried to write the chapters in an independent and self-contained way. Chapter 1 gives a general introduction to the topics discussed in this Thesis. Chapters 2 and 4 review the main general concepts that are important for understanding the further results. The results of the research are described in Chapters 3, 5, and 6 . Chapters 3 and 5 have the subject of applications of topology in condensed-matter physics. Starting from Chapter 5, we discuss topological solitary waves in classical field theory in various inhomogeneous settings, whereas in Chapter 6 we focus on problems that might have influence in biology or medicine in the future.

At the end of the Thesis, the reader will find the bibliography, the summaries in English and Dutch, acknowledgments, and the Curriculum Vitae of the author.

Mariia Lizunova

May 2020, Utrecht 



\section{List of publications}

This Thesis is based on the following publications:

$\diamond$ "Visualizing the connection between edge states and the mobility edge in adiabatic and nonadiabatic topological charge transport"

M. A. Lizunova, F. Schreck, C. Morais Smith, and J. van Wezel

Phys. Rev. B 99, 115114 (2019), [arXiv:1811.06222 [cond-mat.str-el]].

$\diamond$ "Visualizing topological transport in charge ordered materials"

M. A. Lizunova, C. Morais Smith, and J. van Wezel

J. Phys.: Conf. Ser. 1189, 012015 (2019).

$\diamond$ "Visualizing topological transport"

M. A. Lizunova, S. Kuyper, B. Meijer, A. Silva, and J. van Wezel

is accepted to Am. J. Phys., [arXiv:1905.03668 [cond-mat.mes-hall]].

$\diamond$ "Kinks and realistic impurity models in $\varphi^{4}$-theory"

M. A. Lizunova, J. Kager, S. de Lange, and J. van Wezel

sumbitted to JHEP, [arXiv:2007.04747 [nlin.PS]].

$\diamond$ "An introduction to kinks in $\varphi^{4}$-theory"

M. A. Lizunova and J. van Wezel

sumbitted to SciPost Phys. Lect. Notes.

$\diamond$ "Solitary wave propagation in media with step-like inhomogeneities"

M. A. Lizunova and O. Gamayun

In progress. 


\section{Contents}

$\diamond$ "Emergence of oscillons in kink-impurity interactions"

M. A. Lizunova, J. Kager, S. de Lange, and J. van Wezel

In progress.

Other publications to which the author has contributed:

$\diamond$ "Kink interactions in the $(1+1)$-dimensional $\varphi^{6}$ model"

V. A. Gani, A. E. Kudryavtsev, and M. A. Lizunova

Phys. Rev. D 89, 125009 (2014), [arXiv:1402.5903 [hep-th]].

$\diamond$ "Kink excitation spectra in the $(1+1)$-dimensional $\varphi^{8}$ model"

V. A. Gani, V. Lensky, and M. A. Lizunova

JHEP 8, 147 (2015), [arXiv:1506.02313 [hep-th]].

$\diamond$ "Excitation spectra of solitary waves in scalar field models with polynomial self-interaction"

V. A. Gani, V. Lensky, M. A. Lizunova, and E. V. Mrozovskaya

J. Phys.: Conf. Ser. 675, 012019 (2016), [arXiv:1602.02636 [hep-th]].

$\diamond$ "Scalar triplet on a domain wall"

V. A. Gani, M. A. Lizunova, and R. V. Radomskiy

J. Phys.: Conf. Ser. 675, 012020 (2016), [arXiv:1602.04446 [hep-th]].

$\diamond$ "Scalar triplet on a domain wall: an exact solution"

V. A. Gani, M. A. Lizunova, and R. V. Radomskiy

JHEP 04, 043 (2016), [arXiv:1601.07954 [hep-th]].

$\diamond$ "Search for long-living topological solutions of nonlinear $\varphi^{4}$ field theory"

A. E. Kudryavtsev and M. A. Lizunova

Phys. Rev. D 95, 056009 (2017), [arXiv:1610.09966 [hep-th]]. 


\section{Introduction}

Over the past decades, physicists were creative in coming up with mathematical models for actual observed phenomena, in which topological properties take a central role in understanding the problem. Systems with different types of topological order have different robust properties. These properties can only be changed by means of a quantum phase transitions towards a system with another type of topological order. The ideas of topology, then, became essential as basic organizing principles from condensed-matter physics to biology. The main purpose of this Thesis and, in particular, of this chapter is to provide a brief overview to a few examples of applications of topology as a powerful tool.

Let us start with an example in condensed-matter physics. Nowadays, the integer quantum Hall effect is understood to be a part of a much larger class of topological insulators [1-8]. However, in 1980 its experimental discovery by Klaus von Klitzing in GaAs [9] made this two-dimensional material a first example that has an insulating bulk, but metallic edge states. The topological nature of this topological insulator is most clearly seen through the phenomenon of quantized adiabatic particle transport from one edge to the opposite side of these systems, precisely given by the bulk Chern number [10-12]. The topological pump brings together all the main players in the modern understanding of topological matter: quantized conductance, topological quantum numbers, edge states, robustness to disorder, and the bulk-boundary correspondence [13]. One of the examples of this pump is a thought experiment in Laughlin's integer quantum Hall cylinder [14]. However, how precisely the electrons are transferred between edges as quanta of flux are threaded through Laughlin's integer quantum Hall cylinder remains difficult to visualize, despite some attempts $[15,16]$. In Chapter 3 we use that the Harper's equation [17-19] can be interpreted as a tight-binding model for the integer quantum Hall effect in two dimensions and as a family of mean-field descriptions for monatomic commensurate charge-density wave in one dimensions. This correspondence allows us to visualize the detailed dynamics of the electron flow during a single pumping cycle of the charge-density wave state, which is difficult to do directly in the quantum 


\section{Introduction}

Hall setup by using adiabatic and nonadiabatic transport protocols. We illustrate modes of topological transport and discuss possible realizations in the context of ultracold atoms and photonic waveguide experiments.

Another nice example of the importance of topology arises in connection with Josephson effect [20, 21]. This is a phenomenon of an electric current, which flows without any voltage applied, across two layers of superconducting material separated by a thin insulating layer. This device is known as Josephson tunnel junction. These experimental setups $[22,23]$ over the last years became one of the most successful tests for nonlinear theories. Specifically, a model based on the perturbed sine-Gordon equation describes the internal dynamics of the junction, and the magnetic flux quantum called Josephson vortex or fluxon plays a role of topological solitary wave. This wave, as in other integrable systems, moves with a constant velocity and shape and carrys a topological charge that is analogous to the Chern number. However, in nature, plasma waves are generated because of inhomogeneities in the junction [24], and the soliton may experience a modification in evolution. Thus, it is interesting to study inhomogeneities that may affect the most peculiar property of the sine-Gordon model, namely its integrability. To make the results more meaningful, it is better to compare the influence of an inhomogeneity on integrable and on nonintegrable models. In order to do this, we adapt the well-known $\varphi^{4}$ theory, which found its applications in many places, e.g., from Ginzburg-Landau phenomenological theory of second-order phase transitions [25] to the Higgs mechanism [26]. In general, the soliton/kink-impurity interactions for a single impurity are well-studied in both sine-Gordon [27-31] and $\varphi^{4}[32-34]$ systems in $(1+1)$ dimensions, mostly by including an extra potential barrier or well directly in the equation of motion $[33,35,36]$. Nevertheless, one can introduce the impurity through the metric of background space-time [31, 37, 38], or even consider an extended inhomogeneity in space [39]. In Chapter 5, we consider a spatial steplike nonuniformity that is represented by different coupling constants. We study the influence of this type of inhomogeneity on the behavior of one solitary wave, namely a soliton in the integrable sine-Gordon theory and a kink in the nonintegrable $\varphi^{4}$ model. We observe how the waves are accelerated or decelerated, attracted or reflected, depending on the strength of the coupling constant. We first observe in numerical simulations, and then show analytically that most of results for this type of inhomogeneities are model-independent, meaning that the integrable structure of the sine-Gordon model is irrelevant. This robustness is rooted in the topological character of the soliton and the kink. One may consider an inhomogeneous halfspace as a limit case of a Gaussian impurity, which we discuss in Chapter 6, but with infinite width.

Now, let us discuss an application of the theories investigated here in biological system. Recently, it was found that the kinks of the $\varphi^{4}$ model may be useful in the description of proteins [40-42]. The latter [43] is a one-dimensional chain of amino acids (the primary structure). The proteins perform specific functions in 
the cells. However, they usually perform specific functions in the cells only if they have been correctly folded in three dimensions (the tertiary structure). Nowadays, the "protein folding problem"[44-46] is a very relevant research field: only correctly folded proteins may perform properly their functions, thus, keeping the living organism healthy and alive, while proteins without normal native structure may cause diseases [47], e.g., cancer, Alzheimer's disease, and amyloidosis. The first one is characterized by uncontrolled cell division usually caused by mutations that lead to disrupted activity of proteins, many of which may be abnormally folded [48-50]. The second one may be caused by misfolded amyloid-beta protein in the brains [51]. The last is a group of diseases with abnormal proteins (amyloid fibrils [52]) build up in tissue and organs $[53,54]$. Thus, the "protein folding problem" is a hot topic, and there are two important aspects of it: how to predict or explain the tertiary structure of the proteins, and how one could correct it when they are folded incorrectly. Using the gauge field theory approach, it was recently shown [40] that a protein may be schematically represented by a sequence of point-like vertices with torsion and bending angles in between each of them (the angles correspond to kinks in a one-dimensional classical $\varphi^{4}$ field theory). Later [41, 42], an overlay of a native structure of the protein that was reproduced from real data from the Protein Data Bank and an approximate structure reconstructed by a superposition of kinks on the example of Myoglobin yielded fantastic results. The gauge field theory approach reproduces the protein structure up to $1 \AA$ [41, 42], which shows the efficiency of this method. In Chapter 6, we focus on the behavior of a single kink interacting with different types of localized disorder, described by a Dirac delta, a Gaussian, and a Lorentzian functions. We find how the strength and the width of the impurities may affect the movement, causing resonances or capturing the kink, for example. We believe that the obtained results might bring benefits to the control of protein folding in the future, thus, to make a step forward to solve the "protein folding problem". An indication of this is that drugs are used to tackle diseases like Alzheimer's which rely on the folding of proteins. Intrinsically disordered proteins (IDP) are the type of proteins that usually act as targets in such molecular drug therapeutic strategies [55]. Often, those drugs are designed, for example, to bind lysine and arginine, perturbing aggregation and suppressing fibril formation [56]. One of the example of a target-drug couple is the IDP protein p53, that plays a crucial role in human cancer, and the inhibitor nutlin-3 that targets and binds the p53-MDM2 complex [57, 58].

As mentioned in Ref. [42], the effective approach to the modeling of the protein backbone structure by a superposition of kinks not only reproduces the protein structure with high precision [41, 42], but also the dynamical properties of the protein chains may be defined by the corresponding kinks dynamics. Thus, it was natural to extend the kink-impurity interactions, described in the first part of Chapter 6 . In the second part of Chapter 6 we add a second wave, the antikink, and consider the evolution of a kink-antikink pair with a localized Gaussian-like impu- 


\section{Introduction}

rity in between them. In the absence of the impurity, the effect of multi-solitary waves dynamics in the nonlinear Klein-Gordon equation attracted a lot of attention [59, 60]. Particularly, collisions in the framework of $\varphi^{4}$ theory are peculiar, because the kink and the antikink cannot just pass through each other and maintain their shape, as in the sine-Gordon model [59]. The reason is that they interact and quasi-breather [61] or resonance phenomena [60-63] may occur. The dynamics may be studied by numerically solving the equation of motion [61], as well as with a help of the collective coordinate approximation [62, 64-68], or even simulated in a real experiment using a mechanical system consisting of a ball rolling along a specially-designed landscape, to model the resonances [69]. However, to the best of our knowledge, the interaction of this pair with an impurity was not studied earlier. In contrast to kink-impurity interactions, when resonances are observed only for an attractive disorder, we see here new cases of resonances for a repulsive impurity. We also obtain a quasi-long-lived solution with a small amplitude called an oscillon (a Gaussian envelope), which was not observed in earlier calculations for the classical $(1+1)$-dimensional $\varphi^{4}$ model, but only used as an artificial initial condition [70, 71]. We discuss an osillon-oscillon bound state, the presence of the oscillon resonances, and compare these phenomena to the one observed in the sinh-deformed $[72,73]$ $(1+1)$-dimensional $\varphi^{4}$ model [74].

Finally, we would like to point out that this work was started in autumn 2016 in one world and is finishing in spring 2020, during the COVID-19 pandemic, in altered living conditions. One of the main ideas of this work is to show how the solution of certain problems may help in the most unexpected areas of not only physics, but also science as a whole. This is exactly what society needs nowadays: fast graceful solutions. Topology like we show through the whole text, might be one of those powerful tools to achieve them. 


\section{Introduction to topological trans- port}

\subsection{Introduction}

Topology has, over the past decades, taken center stage alongside symmetry as one of the basic organizing principles of condensed-matter physics. As with symmetry, the predictive power associated with topology can be enormous.

The experimental discovery of the integer quantum Hall effect (IQHE) in GaAs [9] in 1980 by Klaus von Klitzing (1985, Nobel prize), revealed the first example of a two-dimensional $(2 D)$ material that has an insulating bulk, but metallic edge states. In the IQHE [9], e.g., knowing the integer value of a single topological quantum number allows one to predict the transverse conductance of a $2 D$ electron gas with unlimited accuracy, independently of how the electron gas is realized experimentally $[75,76]$. Predictions of the transverse conductance have been verified to one part in a billion, and in fact, the Quantum Hall Effect serves as a standard for resistance calibration [77].

Nowadays, the IQHE is understood to be part of a much larger class of topological insulators (TIs) [1-8]. The typical implication of an insulator being topological is the presence of conducting states at its edges. The topological nature of these edge states is seen most clearly through the phenomenon of quantized adiabatic particle transport, more commonly known as topological transport or topological pumping $[10,11]$. Its idea is easily formulated. By periodically changing some driving force, particles are transferred from one edge of the TI to the opposite side. This transport is quantized, in the sense that for every period of the pumping cycle, precisely an integer number of particles will move between edges [10, 12]. It is topological because the discrete number of relocated particles is independent of 


\section{Introduction to topological transport}

the details of both the system and the driving. As long as the driving is adiabatic, and the system remains insulating, the number of transferred particles will always be the same [78]. In fact, the topological nature of the pumping can also be seen as an example of the celebrated bulk-boundary correspondence, since the number of particles relayed between opposing edges precisely equals the integer topological quantum number characterizing the bulk TI $[14,75]$. This correspondence between the bulk topological invariants and the dynamics of edge states has been put forward by Laughlin $[14,75,79,80]$. It shows that adiabatically threading a single AharonovBohm flux quantum through the interior of an IQHE cylinder [14, 79], results in a quantized number of electrons moving from one edge of the cylinder to the opposite. The amount of charge transported is given precisely by the bulk Chern number, defined as an integral over the $2 D$ Brillouin zone (BZ) [12]. The resulting cyclic charge transfer may be regarded as a dynamical manifestation of the IQHE [81] and has been observed in a Corbino geometry $[15,16]$.

Topological pumps thus bring together all the main players in the modern understanding of topological matter: quantized conductance, topological quantum numbers, edge states, robustness to perturbations, and the bulk-boundary correspondence [13, 82]. More generally, it is an example of the type of quantized adiabatic particle transport, or topological charge pumping, first proposed by Thouless $[10,12,75,83]$. Experimentally, topological pumps have been realized in coldatom $[84,85]$ and single-spin [86] systems, and they attract attention in both adiabatic $[87,88]$ and nonadiabatic $[89,90]$ regimes.

The theoretical understanding of the role of topology underlying the IQHE [75, 76], Fractional Quantum Hall Effect [91, 92], Quantum Spin Hall Effect [3, 93], and more generally TIs and other forms of topological matter $[4,94]$ is one of the corner stones of modern condensed-matter physics [6, 95]. Different topological classes emerge depending on whether time-reversal, particle-hole, and chiral symmetries are present or not [96, 97], and may be further refined using lattice symmetries [78, 98101]. Moreover, topological order has been suggested to open the way towards various applications, including dissipationless topological transport, fault-tolerant quantum computation, and the engineering of spin liquid phases of matter $[6,95]$.

\subsection{Integer quantum Hall effect}

In this section, we revise the main features of the IQHE. The reader familiar with these concepts might wish to skip it.

The experimental setup [9] in which the IQHE may be observed is presented in Fig. 2.1. A conductor is connected to a current source in $2 D$. The electrons in the conductor move under the influence of an electric field $\mathbf{E}$ (contributing to a longitudinal current, and resistance $R_{x x}=R_{L}$ ). By applying a homogeneous 
perpendicular magnetic field

$$
\mathbf{B}=\{0,0, B\},
$$

one obtains that the positive charges line up on one side of the conductor, while the negative charges on the other. Thus, the charged particles move transversely to the direction that they would move in the absence of the perpendicular magnetic field B. That is how a transverse, or so-called Hall, current $R_{x y}=R_{H}$ appears. Now, let us consider this phenomenon in detail.

It is well-known that a charge-carrying particle characterized by the charge $e>0$, mass $m$, and velocity $\mathbf{v}$ moving in $2 D$ in a static perpendicular magnetic field $\mathbf{B}$ experience the Lorentz force,

$$
\mathbf{F}_{L}=-e(\mathbf{v} \times \mathbf{B})
$$

The equation of motion in components then reads as

$$
m\{\ddot{x}, \ddot{y}\}=-\left\{e B v_{y},-e B v_{x}\right\},
$$

or, for example, in the $x$-direction

$$
\ddot{x}+\omega_{c}^{2} v_{y}=0, \text { where } \omega_{c}=e B / m \text {. }
$$

Thus, it rotates with cyclotron frequency $\omega_{c}$ along a circular orbit around the guiding center $r_{c}$ with the cyclotron radius $R_{c}=l_{m} \sqrt{2 n+1}$, where $n$ is an integer and $l_{m}=\sqrt{\hbar / e B}$ is the magnetic length, or the unity length of the IQHE, with the Plank constant $h=2 \pi \hbar$. The magnetic length $l_{m}$ is related to the area $\pi l_{m}^{2}$, which encloses one quantum of magnetic flux $\phi_{0}$.

To go from classical to quantum mechanics within a one-electron picture, one uses the minimal coupling,

$$
\hat{H}=\frac{1}{2 m} \hat{\mathbf{p}}^{2} \Rightarrow \hat{H}=\frac{1}{2 m}(\hat{\mathbf{p}}+e \mathbf{A})^{2},
$$

where $\hat{\mathbf{A}}$ denotes a vector potential generating the magnetic field $\mathbf{B}=\operatorname{rot} \hat{\mathbf{A}}$, and we fix the gauge freedom by choosing the Landau gauge

$$
\hat{\mathbf{A}}=\{0, B x, 0\} .
$$

Then, the energy is given by

$$
E_{n}=\hbar \omega_{c}\left(n+\frac{1}{2}\right), n=0,1,2, \ldots
$$

$n$ is a Landau level (LL) index. Landau quantization is a quantization of the radius 


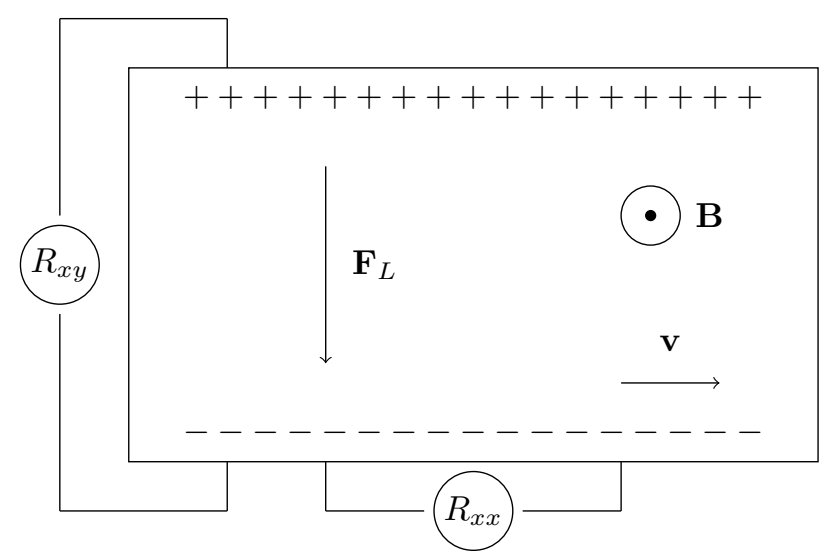

Figure 2.1: Experimental setup for the quantum Hall effect in a $2 D$ electron gas. The arrows show the directions of Lorenz force $\mathbf{F}_{L}$, the velocity of the electron $\mathbf{v}$, and the magnetic field $\mathbf{B}$. The symbols + and - show the charge separation. Here, $R_{x x}=R_{L}$ is the longitudinal resistance and $R_{x y}=R_{H}$ is the Hall resistance.

of the circular trajectory of an electron in a magnetic field B. To see the effects of LLs, i.e., to avoid "jumping" of the electron to the next LL, the thermal energy $k_{B} T$ of the electron, with $k_{B}$ the Boltzmann constant, has to be much lower than $\hbar \omega_{c}$, which is a separation between energy levels $E_{n}$.

It is obvious that in a real case there is more than one electron. For a sample that is finite in the $x$ - and $y$-directions, with length $L$ and width $W$, the density of states in each LL reads

$$
n_{\phi}=\frac{B A}{\phi_{0}}=\frac{\phi}{\phi_{0}}
$$

where $\phi_{0}=h / e$ is the flux quantum, $A=L W$ is the area of the sample, and $\phi=B A$ is the total flux. We define a characteristic ratio $\nu=n_{e} / n_{\phi}$ called the filling factor, which is the ratio between the electron density $n_{e}$ and the flux density $n_{\phi}$ describing how many LLs are filled at a certain value of the magnetic field $\mathbf{B}$.

Classical calculations predict the Hall resistance to depend linearly on the applied magnetic field B [102]. However, experiments have shown that the Hall resistance $R_{H}=B / n_{e}$ e remains constant over a certain range of $\mathbf{B}$, and its value in these plateaus is given in terms of universal constants, namely,

$$
R_{H}=\frac{h}{e^{2}} \cdot \frac{1}{\nu}, \nu=1,2,3, \ldots
$$

It means that $R_{H}$ is quantized and universal, i.e., it does not depend on the partic- 
ular properties of the sample. Here we discuss only integer values of $\nu$ because we consider the IQHE, but in the general case, $\nu$ may be a fraction as well.

To examine the IQHE, we consider that the first $n$ energy levels are totally filled by electrons and the $n+1$ energy level is totally empty. Then, we decrease the magnetic field, which is equivalent to reducing the degeneracy of the filled $n^{t h}$ LL. The electrons, sent to the $n+1$ energy level will populate the valleys of the disorder potential landscape (central panel in Fig. 2.2b). They are localized somewhere in the bulk and do not affect in the global transport characteristics of a sample, measured by the resistance, see lower pannels in Fig. 2.2a and Fig. 2.2b. Therefore, we obtain that $R_{H}$ and $R_{L}$ remain constant despite the change of the magnetic field $B$. This is the origin of the plateau in the Hall resistance.

If one continues to decrease the magnetic field, the regions of the potential landscape in the $n^{\text {th }}$ LL occupied by electrons will become larger, and eventually percolate across the sample, see Fig. 2.2c. At this moment, the longitudinal resistance increases and the Hall resistance is no longer quantized, going from the current plateau to the next lower plateau.
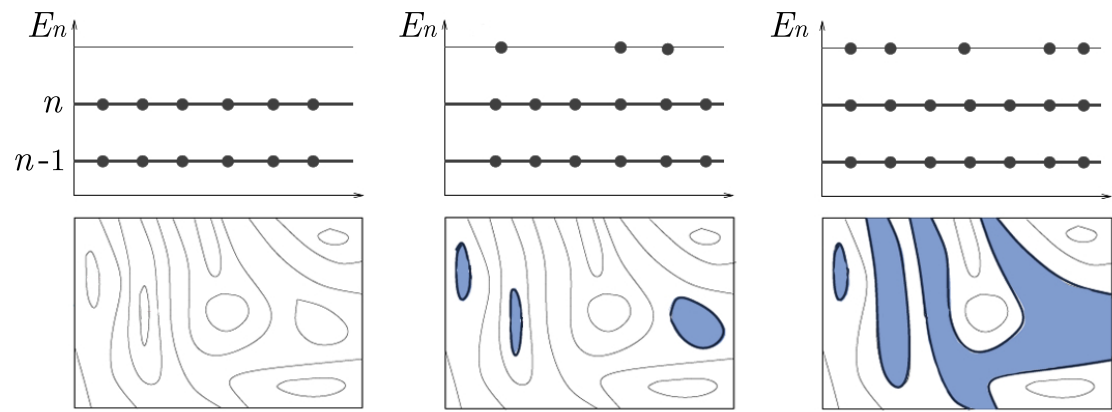

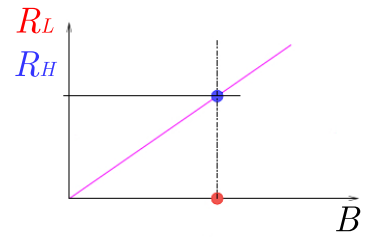

(a)

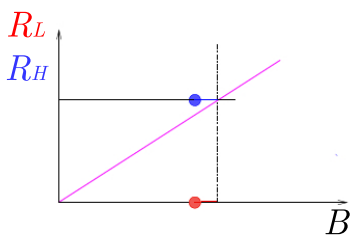

(b)
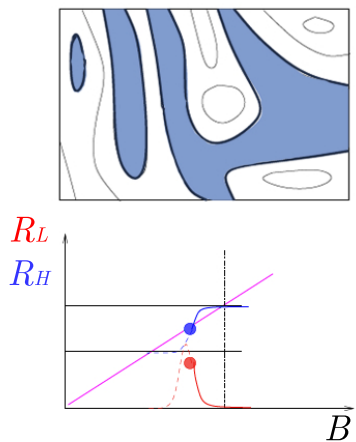

(c)

Figure 2.2: The influence of impurities in IQHE. First line: the filling of the LLs. Second line: the impurity potential landscape of the last partially filled level. Third line: the longitudinal resistance $R_{L}$ (red color), the Hall resistance $R_{H}$ (blue color), and classical prediction for $R_{H}$ (purple color) as a function of the magnetic field $B$. Adapted from [102].

In practice [9], the plateau transition in the Hall resistance $R_{H}$ is accompanied by 


\section{Introduction to topological transport}

a peak in the longitudinal resistance $R_{L}$, which is shown schematically in Fig. 2.3. Experiments show that for the observation of the IQHE in a $2 D$ electron gas, the temperature has to be around $T \approx 1 K$ and the magnetic field as strong $B \approx 1 T$. The last, but important as well, condition is the presence of impurities in the sample. A total absence of impurities will lead to the classical Hall effect; the Hall resistance $R_{H}$ will not be quantized and will linearly depend on the magnetic field $B$. However, a too large number of impurities in a sample broaders the LLs. Thus hampering the observation of its discrete character, which is essential for the IQHE.

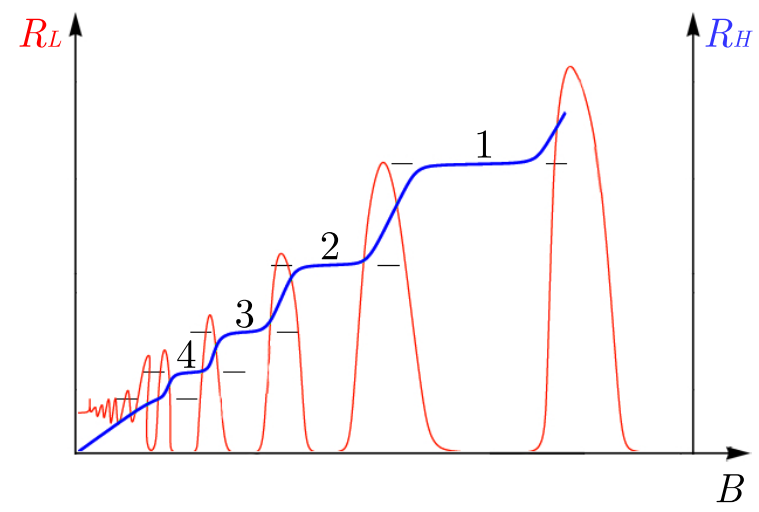

Figure 2.3: Schematic curve for the IQHE. The resistances $R_{L}$ (red color) and $R_{H}$ (blue color) are represented as a function of the magnetic field $B$.

\subsection{Dynamics in Laughlin's IQHE cylinder}

In the semi-classical picture of the IQHE, electrons in a $2 D$ electron gas are confined to the cyclotron orbits that are much smaller than the spatial extent of the system. If the electron gas is confined to the surface of a torus (periodic boundary conditions in all directions), the system will be completely insulating. Only when we look at the IQHE on a surface with boundaries, like a finite sheet or a cylinder, charge transport is possible. This is the so-called Laughlin's Gedankenexperiment. We consider the $2 D$ electron gas in the IQHE regime on a cylinder. We assume that we can thread a flux through the cylinder, and this flux can be changed adiabatically (Thouless pump) by an entire magnetic flux quantum $\phi_{0}$. The change of flux changes of the vector potential. This change, however, does not modify the energy eigenvalues. This means that the physical properties of the system are the same as before. When the Fermi energy lies within a band of localized states, the number of occupied localized states does not change. That means that it is only possible to obtain a 
change in the occupation of the edge states. Hense, electrons can be transferred from one edge to another. For $p$ particles with charge $q$ transferred from one edge to another, one obtains the quantization of the Hall resistance $\sigma_{x y}=p q / \phi_{0}$ [14]. This conductance is strictly quantized, topological in nature, and can be calculated from a bulk topological invariant $[14,75]$. This is the manifestation of the celebrated bulk-boundary correspondence.

Despite its status as an archetype of topological transport, the detailed dynamics of exactly how electrons are transferred between edges as a quantum of flux is threaded through Laughlin's IQHE cylinder remains difficult to visualize. The straightforward plotting of electronic wave functions for arbitrary flux values throughout the pumping cycle is hampered by the necessity to choose a gauge. This should be contrasted to the situation in the IQHE model, where the Laughlin argument [14] provides the direct analog of the Thouless pump [12]. There, configurations in which the flux differs by a value that is not a multiple of the flux quantum, cannot be directly compared to one another because the gauge-invariant momentum is flux-dependent. It, thus, remains unclear how charges are actually transported in real space from one edge to another as a function of real time. Finally, we want to come up with a system, in which the transport visualization may be realized both theoretically and experimentally.

We remedy this situation by presenting a particular topological pumping process that emphasizes the roles of the key players (the topological invariant, the pumping process, and the connection between opposing edges) and that allows for a straightforward visualization of the particle transport throughout the pumping cycle. In order to solve this problem, we use a well-known family of one-dimensional $(1 D)$ charge-ordered systems that can be precisely mathematically mapped onto the $2 D$ IQHE setup [13], and allows for the mathematical identification of edge state dynamics. The details of the mapping will be discussed later, after the presentation of the model. This will allow us to directly visualize the topological transport as electrons flow from one edge of the system to the other, and thus clarify the nature of the transport in both the charge-ordered and IQHE systems. 



\section{Visualizing topological transport in charge-ordered materials}

The detailed dynamics of topological charge transport in the integer quantum Hall cylinder remain difficult to visualize, due to the presence of gauge symmetry. Here, we exploit the reduced gauge freedom in one-dimensional charge-density waves system subject to an external driving force, to demonstrate details of their topological pumping. A close inspection of the instantaneous eigenstates of a particular mean-field charge-ordered model reveals an interplay between topological edge states and the mobility edge. This allows us to visualize adiabatic (Thouless) pumping and nonadiabatic transport protocols in general charge-ordered materials and related topological systems. In the end, we discuss possible experimental setups, where these protocols can be implemented.

\subsection{Introduction}

The ability to pump quantized amounts of charge is one of the hallmarks of topological materials. An archetypical example is Laughlin's gauge argument for transporting an integer number of electrons between the edges of a quantum Hall cylinder upon the insertion of a magnetic flux quantum. This is mathematically equivalent to the equally famous suggestion of Thouless' that an integer number of electrons are pumped between two ends of a one-dimensional quantum wire upon sliding a charge-density wave (CDW) over a single wave length. We use the correspondence between these descriptions to visualize the detailed dynamics of the electron flow during a single pumping cycle, which is difficult to do directly in the quantum Hall setup theoretically and experimentally as well.

Here, we find a close correspondence between topological edge states and the mobility edges in CDW, quantum Hall, and other topological systems. We illus- 
trate this connection by describing an alternative, nonadiabatic mode of topological transport that displaces precisely the opposite amount of charge as compared to the adiabatic pump. We also discuss possible experimental realizations in the context of ultracold atoms and photonic waveguide experiments.

The outline of this Chapter is the following: in Section 3.2, we introduce the CDW, and in Section 3.3 we present the mapping between integer quantum Hall effect (IQHE) and CDW. In Section 3.4 we consider an adiabatic protocol in CDW, then, in Section 3.5, we explain the mobility edge, followed by a nonadiabatic transport in Section 3.6. Afterwards, in Section 3.7 we discuss a possible experimental implementations. Finally, in Section 3.8, we conclude.

\subsection{Charge-density waves}

To be concrete, we consider a CDW on a finite chain of $N$ atoms (sites) described by the Hamiltonian:

$$
H=-t \sum_{j=1}^{N-1}\left(\hat{c}_{j}^{\dagger} \hat{c}_{j+1}+\hat{c}_{j+1}^{\dagger} \hat{c}_{j}\right)+V \sum_{j=1}^{N-1} \hat{c}_{j}^{\dagger} \hat{c}_{j} \hat{c}_{j+1}^{\dagger} \hat{c}_{j+1}+\sum_{j=1}^{N} \zeta_{j} \hat{c}_{j}^{\dagger} \hat{c}_{j} .
$$

Here, $t>0$ signifies the likelihood for an electron to tunnel between neighboring sites, $V$ is the strength of the nearest-neighbor Coulomb interaction, and $\zeta_{j}$ is a random potential that describes the effect of impurities. The operator $\hat{c}_{j}^{\dagger}\left(\hat{c}_{j}\right)$ creates (annihilates) a spinless electron at position $x=j a$, where $a$ is the lattice constant. The filling factor $n=p / q$ is a co-prime fraction, such that $N n$ is the total number of electrons in the system. As usual, we assume the system to be charge neutral in total and ignore any ionic charges.

We use the mean-field ansatz (see Appendix 3.A)

$$
\left\langle\hat{c}_{j+1}^{\dagger} \hat{c}_{j+1}+\hat{c}_{j-1}^{\dagger} \hat{c}_{j-1}\right\rangle \approx 2\left\langle\hat{c}_{j}^{\dagger} \hat{c}_{j}\right\rangle=A \cos (Q j a+\phi)
$$

for the particle density [103]. This ansatz defines the CDW amplitude $A$, the propagation vector $Q=n 2 \pi / a$ of the charge-density modulations, as well as the phase $\phi$ that determines the position of the CDW with respect to the lattice. The interaction term becomes [13]

$$
\sum_{j} V A \cos (Q j a+\phi) \hat{c}_{j}^{\dagger} \hat{c}_{j}
$$

Substituting the interaction back into Eq. (3.1) yields the full mean-field Hamiltonian that one can immediately put into matrix form: 


$$
\begin{gathered}
\hat{H}_{\mathrm{MF}}=\left(\hat{c}_{j=1}^{\dagger}, \hat{c}_{2}^{\dagger} \ldots \hat{c}_{N}^{\dagger}\right) h\left(\begin{array}{c}
\hat{c}_{1} \\
\hat{c}_{2} \\
\vdots \\
\hat{c}_{N}
\end{array}\right), \\
h=\left(\begin{array}{cccccc}
\epsilon_{1} & -t & 0 & \ldots & 0 & -\tilde{t} \\
-t & \epsilon_{2} & -t & 0 & \ldots & 0 \\
0 & -t & \ddots & \ddots & & \vdots \\
\vdots & 0 & \ddots & & & 0 \\
0 & \vdots & & & & -t \\
-\tilde{t} & 0 & \ldots & 0 & -t & \epsilon_{N}
\end{array}\right) .
\end{gathered}
$$

Here,

$$
\epsilon_{j}=V \rho(j)+\zeta_{j}=A V \cos (Q j a+\phi)+\zeta_{j},
$$

where $\rho(j) \equiv\left\langle\hat{c}_{j}^{\dagger} \hat{c}_{j}\right\rangle$. The introduced elements $\tilde{t}$ at the corners of the matrix given by Eq. (3.5) may be used to implement different boundary conditions. An open chain with nothing attached to the edges is described by using $\tilde{t}=0$. An intermediate case of periodic boundary conditions, where the edges are connected via a weak link, corresponding to an additional wire in an experimental implementation, may be modeled by taking $0<\tilde{t} \ll t$, or $\tilde{t}=t$.

\subsection{Mapping between IQHE and CDW}

Harper's equation, and more generally, a Mathieu equation [17-19], can be interpreted both as a tight-binding model for the IQHE in $2 D$, and as a family of mean-field descriptions for monatomic commensurate charge-density waves (CDW) in $1 D[13,104]$. The filling of the charge-ordered system, and hence its CDW periodicity, for example, can be taken to correspond to the magnetic field strength perpendicular to the surface of a quantum Hall cylinder, which determines the filling of its Landau levels $[13,19]$. Under the same mapping, the phase $\phi$ of the CDW order parameter then corresponds to a flux threading the quantum Hall cylinder [14], while the spatial coordinate along the CDW chain is directly related to the spatial coordinate in the periodic direction of the $2 D$ cylinder. The mapping is indicated schematically in figure 4.1 .

Upon varying the parameter $\phi$ adiabatically from 0 to $2 \pi$, the CDW slides along the $1 D$ chain over precisely one wave length. The flux threading the IQHE system is increased by one quantum under the same variation. When introducing edges, we 


\section{Visualizing topological transport in charge-ordered materials}

thus expect to find adiabatic transport of a quantised number of electrons from one side of the system to the other in both cases. In this way, the mapping relates the quantized adiabatic particle transport (Thouless pumping [12]) in charge-ordered systems to the topological transport between edge states upon insertion of a flux quantum in a quantum Hall cylinder (Laughlin's gauge argument [14]).

The bulk of both the CDW and IQHE systems is insulating, and only the edge states cross the Fermi level. By solving Harper's equation on a cylinder, the spectrum can be plotted as a function of momentum in the periodic direction. It consists of bulk bands and isolated topological edge states crossing the gaps between them. The edge states are protected, in the sense that the number of edge channels cannot be modified, as long as the bulk of the system remains gapped [14]. In a CDW system the periodic direction is given by the mean-field value of the phase variable $\phi$, as shown in figure 4.1. Each value of the phase then corresponds to a single realisation of a CDW on the chain, which may or may not host edge states. The combined spectrum of the family of CDWs containing all values of $\phi$ coincides with that of the IQHE cylinder.

For periodic boundary conditions, i.e. $\tilde{t}=t$ and $\zeta_{j}=0$, we perform Fourier transformation (see Appendices 3.B and 3.C) in Eq. (3.5) to obtain

$$
\begin{aligned}
\hat{H}_{\mathrm{MF}} & =\sum_{k}\left(\hat{c}_{k+Q}^{\dagger}, \hat{c}_{k+2 Q}^{\dagger} \ldots \hat{c}_{k+q Q}^{\dagger}\right) H_{k}\left(\begin{array}{c}
\hat{c}_{k+Q} \\
\hat{c}_{k+2 Q} \\
\vdots \\
\hat{c}_{k+q Q}
\end{array}\right), \\
H_{k} & =\left(\begin{array}{cccccc}
\epsilon_{k+Q} & \tilde{\Delta} & 0 & \ldots & 0 & \tilde{\Delta}^{*} \\
\tilde{\Delta}^{*} & \epsilon_{k+2 Q} & \tilde{\Delta} & 0 & \ldots & 0 \\
0 & \tilde{\Delta}^{*} & \ddots & \ddots & & \vdots \\
\vdots & 0 & \ddots & & & 0 \\
0 & \vdots & & & & \tilde{\Delta} \\
\tilde{\Delta} & 0 & \ldots & 0 & \tilde{\Delta}^{*} & \epsilon_{k+q Q}
\end{array}\right),
\end{aligned}
$$

where $k$ is the wave number with a condition $k+q Q=k$ and $\tilde{\Delta}=V A e^{i \phi}$. Numerical diagonalization of Eq. (3.8) yields the energy spectrum, or band structure, for any given value of the CDW phase $\phi$, as shown in Fig. 3.1a. Collecting the energies associated with all possible choices for $k$ and $\phi$ at a given filling fraction $n$ and plotting them as dots in the plane of energy versus filling fraction, yields a version of the famous Hofstadter butterfly spectrum [13, 19, 105] shown in Fig. 3.1b. This form of the Hamiltonian will coincide with Hofstadter's tight-binding description of the 
IQHE [19], if we make the identifications $k \rightarrow k_{x}, Q \rightarrow e B /(\hbar c)$, and $\phi \rightarrow k_{y} a$. Here, $k$ is the momentum in the CDW, and $k_{x, y}$ are the $x, y$-components of momentum in the IQHE. The radial magnetic field $B$ in the IQHE cylinder determines the filling of its Landau levels. Under this mapping, adding flux along the central axis of the IQHE cylinder corresponds to changing the value of the phase $\phi$ in the CDW [14], and thus to sliding the charge-density wave along the chain.

The Hamiltonian itself may be realised as a mean-field description of charge order in $1 D$ chains of aligned orbitals within a three-dimensional material [106], or as an effective description of cold atoms in an optical lattice [13, 107]. Here, we focus first on the topological properties of the theoretical mean-field model, before discussing possible experimental probes of the various emerging modes of topological transport in more realistic settings.

\subsection{Adiabatic transport}

In Laughlin's argument for quantized transport across the IQHE cylinder [14], the electromagnetic gauge structure plays a central role. Although helpful in establishing why the charge transferred between the ends of the cylinder must be integer, the presence of gauge freedom makes it hard to directly visualize the precise dynamics. We know and understand what happens upon insertion of a single flux quantum, but questions like at which value of the flux one edge state becomes unoccupied, and the opposite one occupied, or what the wave function looks like after insertion of only half a flux quantum, are difficult to answer in a gauge-independent fashion. For the CDW system, this problem does not exist. The process corresponding to a flux insertion is the sliding of the CDW by precisely one wave length, and this results in a precisely quantized amount of charge being transferred from one end of the chain to the other (Thouless pumping [12]). The quantized conductivity is determined by the sum of Chern numbers for all occupied bands (in $k, \phi$-space), as in the IQHE. However, in this case we can plot the charge distribution for both bulk and edge states for any value of $\phi$, and hence visualize the topological transport as a continuous process.

As shown in Fig. 3.2, the spectrum and eigenstates can be computed for a specific choice of parameters, including any value of $\phi$. As an example, we show the results for a period-3 CDW with $n=1 / 3, N=63$, and $A V / t=5$. We use this specific example, but note that taking other parameter choices does not qualitatively affect any of the obtained results. The number $N$, however, should be odd.

Among the numerically obtained wave functions in Fig. 3.2, bulk and edge states can be easily distinguished. The bulk wave functions for a CDW with open boundary conditions can be understood as products of plane waves (solutions of a CDW system with periodic boundary conditions) and the eigenstates of a particle-in-abox. The edge states, on the other hand, are exponentially localized on one side of 
3 Visualizing topological transport in charge-ordered materials

(a)

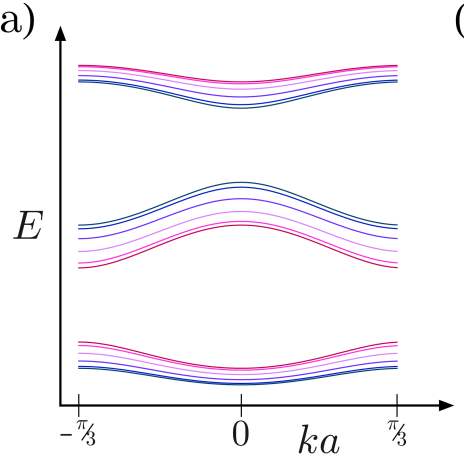

(c)

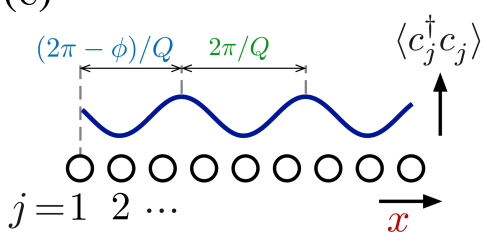

(b)
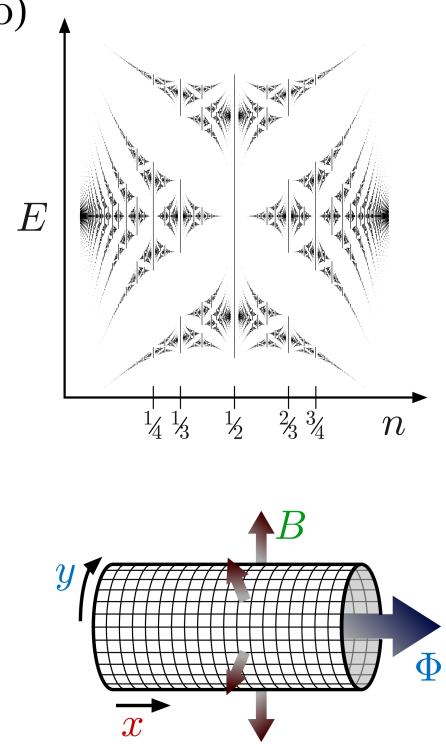

Figure 3.1: (a) Dispersion relation for the mean-field CDW with periodic boundary conditions. Different colors correspond to different values of $\phi$, ranging between zero (purple) and $\pi / 3$ (blue). The whole system is $2 \pi / 3$-periodic, thus $\phi=0$ is equivalent to $\phi=2 \pi / 3 ; 4 \pi / 3 ; 2 \pi$, and $\phi=\pi / 3$ is equivalent to $\phi=\pi ; 5 \pi / 3$. (b) The spectrum of the mean-field CDW as a function of filling fraction. For each $n$, ranges are indicated for all energy eigenvalues found as $k a$ is varied between $-\pi /(3)$ and $\pi /(3)$, and $\phi$ is varied between zero and $2 \pi$. The resulting figure is known as Hofstadter's butterfly and was first found in a tight-binding model for the IQHE. (c) Pictorial representation of the correspondence between the mean-field CDW and a tight-binding model for the IQHE.

the chain.

Upon varying $\phi$, the highest occupied state changes from an edge state localized on one side to that on the opposite side. In the example of $1 / 3$ filling shown in Fig. 3.3, the highest state with $E<0$ at $\phi=0$ is an edge state localized at the right side of the chain $(j=63)$. In the region $\pi / 3 \lesssim \phi \lesssim \pi$, the edge state is adiabatically transformed into a bulk state, until it emerges again as an edge state on the opposite side of the system. At $\phi \simeq 5 \pi / 3$, the highest occupied state discontinuously changes from being localized on the left side of the chain $(j=1)$, to being localized on the right. From the spectrum, it is clear that this behavior 

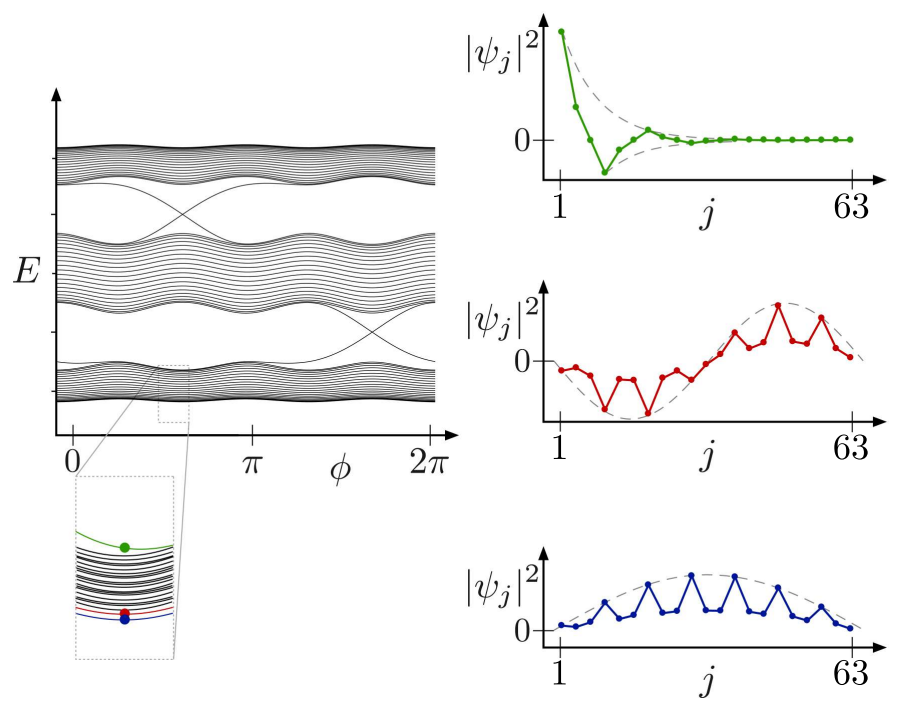

Figure 3.2: The spectrum of the mean-field CDW on an open chain, as a function of the phase variable $\phi$. The real-space wave functions for some fixed value of $\phi$ for the lowest energy state, first excited state, and the first edge state are displayed as well, with colors corresponding to the labels shown in the inset. Also indicated are the exponential and sinusoidal envelopes, which show that the low-energy wave functions are modulated particle-in-a-box states, and the edge state is exponentially localized at position $j=0$.

stems from the two edge states crossing in energy at this point.

Notice that the states displayed in Fig. 3.3 are the highest occupied states at zero temperature for a given value of the phase variable $\phi$. Starting from a phase value $\phi<5 \pi / 3$ and adiabatically sliding the CDW forward, the system will in fact, not stay in the instantaneous ground state. The two edge states crossing within the bulk gap are located at opposite edges of the chain in real space, and any matrix element of local operators that could assist in tunneling across is exponentially small in the chain length. A state on one end of the system can therefore not simply jump to the other end in the way suggested by Fig. 3.3. For a sufficiently long CDW, an adiabatic variation of $\phi$ causes the system to end up in an excited state at $\phi=2 \pi$, with a high-energy edge state occupied and a lower-energy edge state empty, see Fig. 3.4a. The system can only return to its instantaneous ground state, and the topological material can only function as an adiabatic charge pump if the two edges of the CDW chain are connected to one another through some external coupling. 


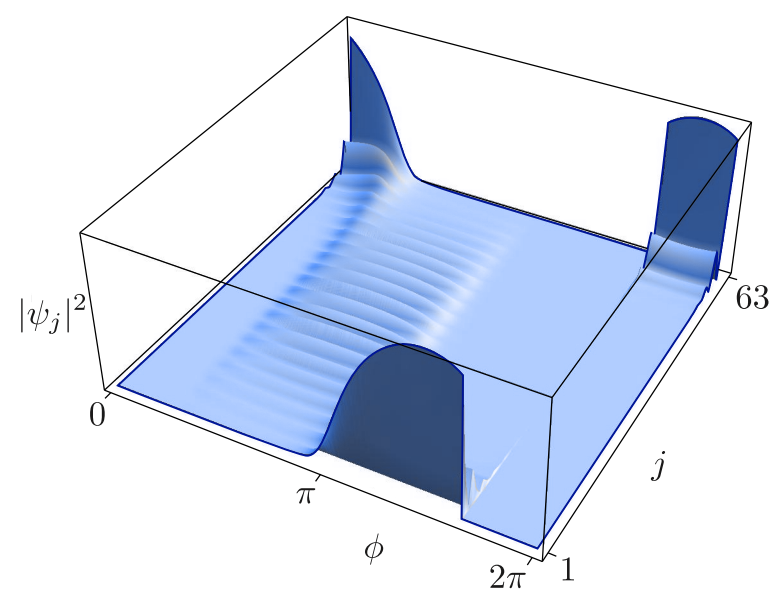

Figure 3.3: The squared amplitude of the highest occupied wave function at $1 / 3$ filling, as a function of phase $\phi$ and position $j$ along the CDW chain. The bulk state between $\phi \approx \pi / 3$ and $\phi \approx \pi$ can be recognized as a particle-in-a-box eigenfunction with additional CDW modulations. It undergoes an avoided crossing at $\phi \approx \pi$ and becomes a left edge state for values of $\phi$ up to $5 \pi / 3$, where it changes abruptly into a right edge state until coming back to $\phi \approx \pi / 3$. If opposing edges of the chain are connected, the abrupt tunneling from left to right will turn into an avoided crossing of its own, whose adiabatic traversal may correspond for example to the physical process of charge being transferred from one edge to another through an intermediary wire.

In fact, in any experimental implementation of a topological quantum pump, one would indeed include a wire connecting the two sides of the system and typically measure the current through the wire as the phase is being varied. Such a connection can be easily included in the simulation as a weak hopping element between the opposing sides of the chain by adding $\tilde{t}$ in Eq. (3.5). It allows the two edge states to interact, and turns their intersection in the spectrum into an avoided crossing by opening up a small energy gap, see Fig. 3.4b. Adiabatic evolution, which retains the instantaneous ground state throughout a pumping cycle, is once again possible.

Thus, adiabatic varying $\phi$ from 0 to $2 \pi$ then results in the transfer of precisely one (the Chern number of the lowest band in $k_{x}, \phi$-space [13], see Appendix 3.D) electron from one side of the system to the other, through the connecting wire, and thus realizes the Thouless pump, or in the IQHE interpretation of the same system, Laughlin's gauge argument. 
(a)
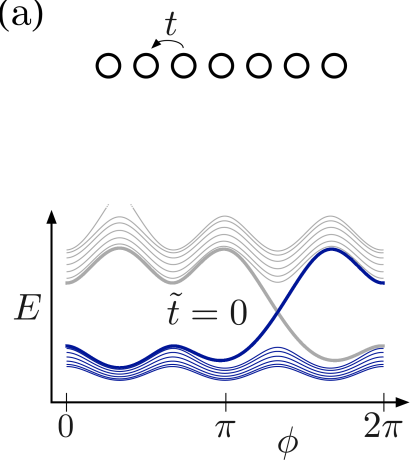

(b)
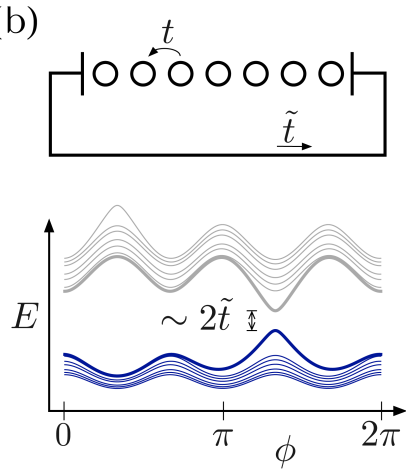

Figure 3.4: (a) The spectrum of the mean-field CDW on an open chain, as a function of the phase variable $\phi$, which may be adiabatically varied from zero to $2 \pi$ as a function of time. Without any connection between the ends of the chain, the many-particle CDW ground state with one fully occupied band of electronic states at $\phi=0$ will adiabatically evolve into an excited state in which the lowest band has one empty state, and the second band has a single electron in it. (b) Upon including a weak connection between edges in the model, which corresponds to the wire that would be used to measure the pumped current in any experimental realization of the adiabatic particle transport, the edge states in the spectrum become gapped. The many-particle ground state now evolves back into itself after a full $2 \pi$ cycle of the phase $\phi$. While doing so, a single electron traverses the external wire (at $\phi \simeq 2 \pi / 3)$ and comes back though the bulk of the CDW (between $\phi \simeq 4 \pi / 3$ and $\phi \simeq 2 \pi)$.

\subsection{Emergence of a mobility edge}

Inspecting the bulk wave functions in the spectrum of Fig. 3.1, two features stand out. First of all, as the edge state enters the bulk band, it does not simply disappear. Tracking, for example, the highest occupied state in Fig. 3.1, it is clear that the state, which was an edge state at $\phi<\pi / 3$, becomes a bulk state at $\pi / 3 \lesssim \phi \lesssim \pi$. The bulk state is the particle-in-a-box state with the highest available number of nodes, dressed with charge-density modulations. A state with the same number of nodes in fact, already existed for $\phi<\pi / 3$, as part of the occupied bulk states. The edge state, therefore, does not evolve into a new bulk state as $\phi$ is adiabatically changed, but rather has an avoided crossing with an existing bulk state and takes over its character. The existing bulk state takes over the character of the edge state 


\section{Visualizing topological transport in charge-ordered materials}

and is pushed down in energy in the process. It then has an avoided crossing with the next bulk state and so on. This pattern is shown schematically in Fig. 3.5.

Close inspection of the wave functions in the spectrum indeed shows a cascade of avoided crossings, witnessed by a remainder of an exponential localization that is visible in bulk states undergoing the avoided crossings. These continue until the edge state reaches precisely the middle of the bulk band. There, it emerges again for an extended range of $\phi$, in the form of another well-known topologically special state, the mobility edge [108]. This isolated critical state was to be expected in the center of the bulk band because the extended bulk states generic to the disordered system are known to be constricted to just a single energy precisely in the center of each (impurity-broadened) LL in the case of the quantum Hall effect [109]. Just like the mobility edge was formed from a left edge state at $\phi \simeq \pi / 3$, it goes through a second series of avoided crossing and reemerges as a right edge state at $\phi \simeq \pi$. The edge states are of the form $\psi(x) \propto \exp (\lambda x)$, with $\lambda$ negative or positive for left or right localized states respectively. The mobility edge naturally connects these two states and has the same shape of the wave function, with $\lambda=0$. This special, totally delocalized wave function is thus a plane wave connecting both sides of the sample. Within the CDW, the plane wave is again dressed by charge modulations, as shown in Fig. 3.6a. Note that for even $N n$, it is possible to have two orthogonal, delocalized wave functions. We consider chains with odd $N n$ here, so that a single mobility edge connects to edge states in both bulk gaps.

The topological nature of the mobility edge only really becomes apparent upon switching on a random impurity potential $\zeta_{j}$ in Eq. (3.1). Notice that in contrast to the IQHE, where the presence of weak impurities is necessary to observe the quantization of the Hall conductivity, in the CDW system topological transport may be detected even with only the Coulomb interaction. Nevertheless, the presence of weak impurities (modeled here with $\left|\zeta_{j}\right| / t<0.2$ ) may be considered, and their effect is much the same as in the IQHE, localizing electronic states at specific locations in the chain. The difference between the mobility edge and other bulk states is now immediately obvious. As shown in Fig. 3.6b, typical bulk states are severely affected by the impurity potential, being amplified and suppressed at random locations. The mobility edge, on the other hand, retains a more or less constant amplitude along the entire length of the chain, as long as the impurity strength is weak. This clearly indicates the topological nature of the mobility edge, and hence its connection with the topological edge states.

\subsection{Nonadiabatic transport}

The connection between edge states and mobility edge may be illustrated by considering a nonadiabatic mode of quantized transport, which exists in addition to the well-known adiabatic Thouless pump. Starting for example with the (red) left edge 

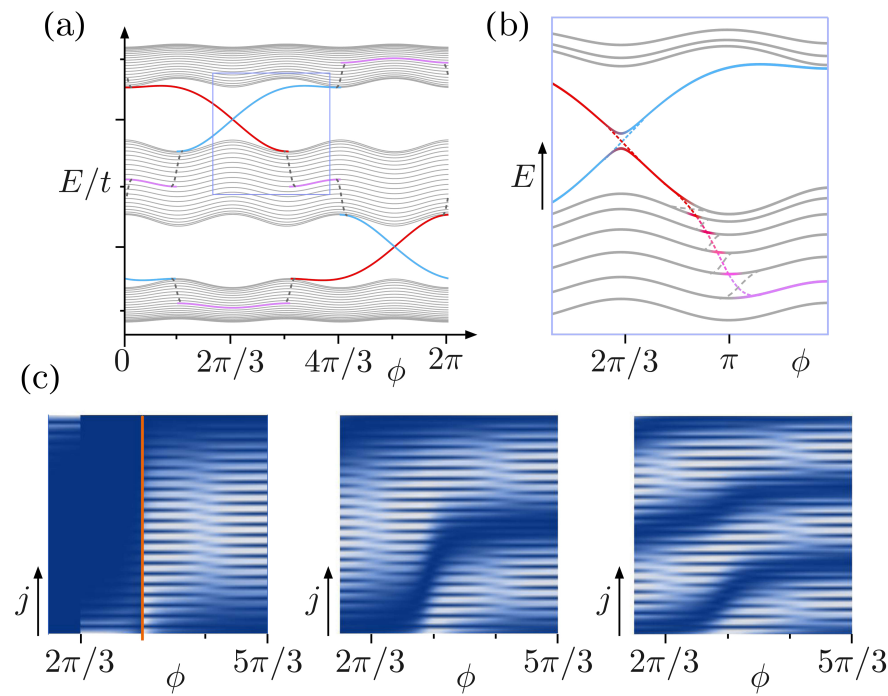

Figure 3.5: (a) Sketch of the spectrum as a function of the phase $\phi$ with all states related to the topological order indicated. Red lines crossing bulk gaps are edge states localized at the left $(j=1)$ end of the chain, blue lines are right $(j=63)$ edge states, and purple lines in the middle of bulk bands represent mobility edges. (b) Schematic close-up view of the crossing of two edge states and of an edge state entering the bulk band. If the two edges are connected they undergo an avoided crossing, which can be either adiabatically traversed by tuning $\phi$ very slowly, or nonadiabatically, by increasing $\phi$ more rapidly in the region of the avoided crossing. As the edge state enters the bulk band, it necessarily undergoes a series of avoided crossings, which ultimately connect the edge state to the mobility edge. (c) Intensity plot of selected wave function amplitudes $\left|\psi_{j}(\phi)\right|^{2}$, for different values of the phase $\phi$. In all plots, dark blue and white indicate low and high amplitudes respectively. The leftmost plot shows the state which (b) starts out as a right edge state (blue line) for low $\phi$, then becomes a left edge state (red line), and after the avoided crossing at $\phi \simeq \pi$ becomes a bulk state (grey line). The orange line indicates that separate color scales are used for the edge and bulk states. The other two panels show the states that start out at low $\phi$ as the topmost state in the lower bulk band and the one below it. The avoided crossings at $\phi \simeq \pi$ can be identified by the changing number of nodes in the wave function. 
(a)

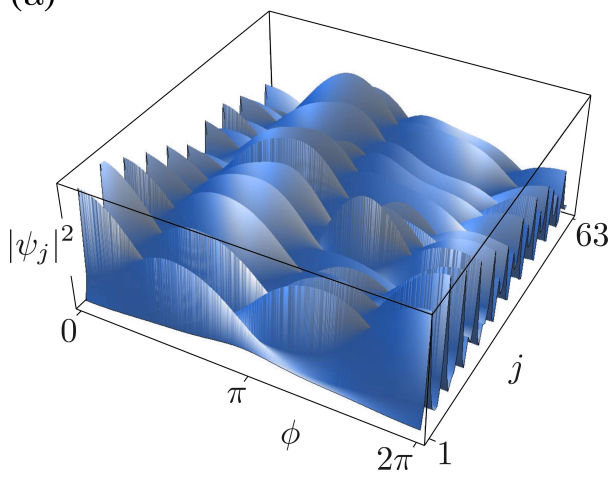

(b)

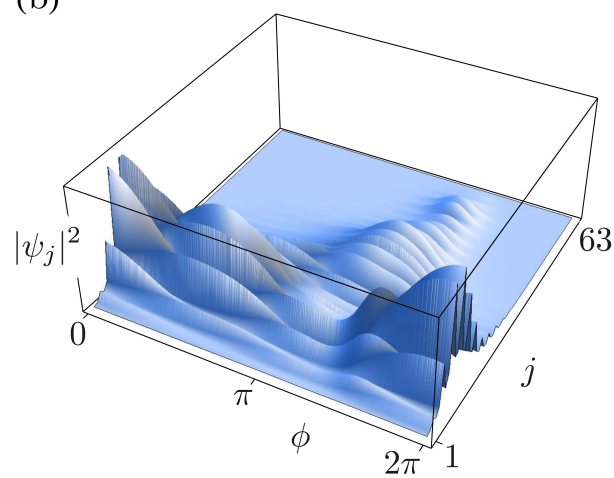

Figure 3.6: Wave functions in the presence of randomly distributed weak impurities $\left(\left|\zeta_{j}\right| / t<0.2\right)$. The delocalized mobility edge in (a) is hardly affected by the impurities, testifying its topological character. The lowest energy state in (b), on the other hand, becomes completely localized at the location of the strongest impurity. For both panels, the same distribution of impurities was used.

state just below $\phi \simeq 5 \pi / 3$ occupied, an induced nonadiabatic evolution that jumps all avoided crossings with both bulk and edge states would result in the highest occupied state going around the full spectrum as shown schematically in Fig. 3.5a. As $\phi$ is increased by $6 \pi$, the highest occupied state has traversed all topologically special states in the entire spectrum and returns to its initial configuration. Stopping the nonadiabatic evolution after $\phi$ is increased by $4 \pi$, however, the highest occupied state will have moved from its initial left edge state to the right edge state in the lowest bulk gap. In the presence of a wire connecting both ends of the chain, we can then either adiabatically increase $\phi$ by another $2 \pi / 3$, or let the system spontaneously relax to its ground state. Both will lead to the excited state going from right to left through the wire. That is, current is carried through the connecting wire in the direction opposite to that of the usual adiabatic topological transport. This nonadiabatic transfer of charge with the same magnitude but in the opposite direction of the usual Thouless pumping is made possible by the edge states and mobility edges forming a connected set of states winding throughout the electronic spectrum.

Generalizing the procedure to systems of any filling, the integer number of charges transferred by the nonadiabatic mode of transport will equal $-C$, with $C$ the total Chern number of all occupied bands, the exact opposite of the $C$ electrons conveyed in the usual Thouless pump. In this sense, it acts as a sort of anti-Thouless pump. Notice that hybrid protocols employing both adiabatic and nonadiabatic driving can 
accomplish the same thing in a much simpler fashion. In such protocols, however, the highest occupied state is in nontopological, localized bulk states for part of the pumping cycle. The purely nonadiabatic process suggested here, in which the highest occupied state jumps all avoided crossings that it encounters, is special because it depends entirely on the special nature and connectivity of the topological edge states and mobility edge states.

The non-adiabatic topological charge pump presented here is intended purely as an illustration of the connection between different types of topological states. Nevertheless, non-adiabatic or hybrid protocols may in some aspects be preferable to purely adiabatic ones in practical implementations of topological pumping. As has been recently pointed out, the presence of a spectrum of unoccupied states imposes much more stringent conditions for achieving adiabaticity than just the time scale of the driving being longer than the inverse gap size [110-112]. Nonadiabatic driving across an avoided crossing, on the other hand, can always be accomplished by sufficiently fast changes of the driving parameters, as long as the overlap between initial and target states is large. We confirmed that this is the case in the model CDW system by achieving an almost complete transfer of occupation across avoided crossings upon ramping up the driving speed.

\subsection{Possible experimental implementation}

To realize the proposed nonadiabatic pumping cycle in experiments, several techniques for imaging the real-space structure of CDW systems can be used.

Scanning tunneling microscopy (STM) for example, can directly observe the amplitude and phase of charge-density modulations induced by a CDW [113-116], as well as charge excess at the end of a chain [117]. More straightforward experimental realizations of quantized transport protocols, however, use ultracold atoms in an optical lattice or photons in a waveguide array. The mean-field Hamiltonian can be constructed with the help of ultracold atoms by projecting a circular optical dipole lattice through a microscope objective [107, 118]. Introducing a weak link between two particular sites in the lattice allows them to act as edges, while the interaction term can be implemented by superimposing a rotating lattice with larger spacing [107]. Variations of the mean-field CDW phase $\phi$ are then implemented by shifting the phase of the rotating lattice with respect to the system edge. After a full driving cycle, the quantized build-up of charge at the edges can be directly observed using the microscope. Photons in a waveguide array are an alternative way of directly simulating the CDW Hamiltonian [117]. Variations of the mean-field CDW phase $\phi$ are then implemented by appropriate variations of the index of refraction. The quantized transport of charge can be detected by injecting photons into the waveguide at one edge and observing the intensity distribution in the waveguides after various propagation distances. The required values for experimental param- 


\section{Visualizing topological transport in charge-ordered materials}

eters in both types of experiments can all be realistically obtained with existing technology. Let us now discuss one of the proposed methods in more detail.

Using ultracold atoms, the mean-field Hamiltonian may be constructed by projecting a circular optical dipole lattice of 63 sites through a microscope objective $[107,118]$, e.g., with a numerical aperture of $\mathrm{NA}=0.8$ [119], and a short wave length of $532 \mathrm{~nm}$ [120], obtaining a waist of the projected Gaussian beams of the order of $0.4 \mu \mathrm{m}$. To ensure that the projected potential is attractive at this wave length, one could use, for example, Sr atoms to load the lattice with. Gaussian beams with a spacing of $0.7 \mu \mathrm{m}$ then result in a potential that closely resembles a sine. The lattice depth will be approximately 0.3 times the depth of one Gaussian beam. The lattice sites can be created by imaging a mask or a pattern from a digital mirror device (DMD) through the objective [107]. In order to confine the atoms in the direction orthogonal to the plane of the ring, an optical lattice can be applied in that direction.

Appropriate values of the model parameters, establishing a Mott-insulator regime with a weak residual tunneling, may be achieved by choosing a lattice depth of about eight recoil energies, or approximately $10 \mathrm{kHz}$. The mean-field energies are then $V=270 \mathrm{~Hz}$ and $t=45 \mathrm{~Hz}$. A weak link may be introduced between two particular sites in the lattice to allow them to act as edges. The strength of the tunneling across the weak link can be adjusted arbitrarily far down from $45 \mathrm{~Hz}$ by increasing the spacing between the two selected sites.

To create and move the CDW, an additional lattice with larger spacing may be added [85]. The required specifics for this additional lattice are much more relaxed than the ones of the primary lattice and can be freely adjusted within a sizeable range. The rotation of the secondary lattice could be achieved by imaging a DMD or a rotating mask onto the atoms. The life-time of the system is limited by off-resonant scattering of the strongest, primary lattice, which for the parameters discussed above, will be over 200 seconds. The experiment should take a few times 63 tunnel times, which is a few times 2.8 seconds, and therefore comfortably fits in the expected experimental lifetime. The system can be prepared in its ground state by using the Mott-Insulator transition. After a full driving cycle, the quantized build-up of charge at the edges can be detected by quantum gas microscopy, which directly measures the parity of the number of atoms in each lattice well.

In other words, the proposed nonadiabatic transport protocol may be done with existing technologies.

\subsection{Conclusion}

In this Chapter, we visualized adiabatic and nonadiabatic topological charge transport in a $1 D$ charge-ordered system, which is well-known to be mapped precisely onto the tight-binding model for the $2 D$ IQHE in the Landau gauge used by Laugh- 
lin to explain topological transport in an IQHE cylinder. The absence of an electromagnetic field in the CDW, and hence of the need to make a gauge choice, enables us to directly compare plots of wave functions at different values of the CDW phase. The topological transport and dynamics of electrons in the CDW chain found here, thus give direct insight into the detailed motion of electronic states in the IQHE as well.

The visualisation of the topological transport brings to the fore an explicit connection between edge states localized at the ends of the chain or cylinder, and the mobility edges residing in the very centre of their bulk states. To illustrate the connection between these two types of topological states, localized and extended, we formulate a purely non-adiabatic protocol that nonetheless results in quantized, topological transport of charge. The number of electrons pumped in a single cycle is precisely equal to that of the well-known adiabatic pump, but they flow in the opposite direction, creating a sort of "anti-Thouless" pump. We expect our conclusions, and in particular the connection between edge states and the bulk mobility edge to apply more generally to disordered topological systems. Any system with a non-trivial Chern or $\mathrm{Z}_{2}$ invariant is guaranteed to have edge states, and similarly mobility edges arise generically in models with disorder-induced localization. A simplified intuitive picture of the connection between the two types of states, suggested by the CDW system studied here, can then be drawn starting from an edge state localized on one side of the system. By evolving this state as a function of some system parameter, such as the CDW phase, it may be adiabatically connected to an edge state on the opposite side of the system. If the evolution is adiabatic throughout, however, a fully delocalized state must generically exist between the two edge-localized extremes.

We discussed experiments on ultracold atoms in an optical lattice, as well as in photonic waveguides, which can test the proposed connection between edge states and mobility edges, as well as the nonadiabatic pumping cycle, using realistic parameter values.

We are indebted to Prof. Dr. Florian Schrek for a quantitative analysis of experimental parameters for the realization of this phenomenon, as described in Section 3.7. 


\section{Appendix}

\section{A The mean-field Hamiltonian}

To find the mean-field description of CDW in $1 D$, we start from the Hamiltonian given by Eq. (3.1) with $\zeta_{j}=0$,

$$
\hat{H}=\sum_{j=1}^{N-1}\left\{-t\left(\hat{c}_{j}^{\dagger} \hat{c}_{j+1}+\hat{c}_{j+1}^{\dagger} \hat{c}_{j}\right)+V \hat{c}_{j}^{\dagger} \hat{c}_{j} \hat{c}_{j+1}^{\dagger} \hat{c}_{j+1}\right\} .
$$

For now, we will use periodic boundary conditions, so that the labels $j$ and $j+N$ correspond to the same site.

The idea behind the mean-field analysis is that we expect to be able to make a reasonable guess for the ground state expectation value of the electron density $\rho(j) \equiv\left\langle\hat{c}_{j}^{\dagger} \hat{c}_{j}\right\rangle$. At the end of the calculation, one may check that the initial guess is indeed consistent with the model we end up with. To see how we can use the fact that we know what to expect for the density operator, first rewrite it as:

$$
\begin{aligned}
\hat{c}_{j}^{\dagger} \hat{c}_{j} & =\left\langle\hat{c}_{j}^{\dagger} \hat{c}_{j}\right\rangle+\hat{f}_{j} \\
\text { with } \hat{f}_{j} & \equiv \hat{c}_{j}^{\dagger} \hat{c}_{j}-\left\langle\hat{c}_{j}^{\dagger} \hat{c}_{j}\right\rangle .
\end{aligned}
$$

This expression defines the fluctuation operator $\hat{f}_{j}$ and does not involve any approximation yet. Assuming that we do have a good guess for the expectation value of the electron density, however, we may assume that the expectation value of the fluctuations $\hat{f}_{j}$ is small, and its square even smaller. We can use this by rewriting the Hamiltonian in terms of the fluctuation operator and then neglecting all terms of quadratic (or higher) order in the fluctuations:

$$
\begin{aligned}
\hat{H} & =\sum_{j=1}^{N-1}\left\{-t\left(\hat{c}_{j}^{\dagger} \hat{c}_{j+1}+\hat{c}_{j+1}^{\dagger} \hat{c}_{j}\right)+V\left(\left\langle\hat{c}_{j}^{\dagger} \hat{c}_{j}\right\rangle+\hat{f}_{j}\right)\left(\left\langle\hat{c}_{j+1}^{\dagger} \hat{c}_{j+1}\right\rangle+\hat{f}_{j+1}\right)\right\} \\
& \approx \sum_{j=1}^{N-1}\left\{-t\left(\hat{c}_{j}^{\dagger} \hat{c}_{j+1}+\hat{c}_{j+1}^{\dagger} \hat{c}_{j}\right)+V\left(\left\langle\hat{c}_{j}^{\dagger} \hat{c}_{j}\right\rangle \hat{f}_{j+1}+\left\langle\hat{c}_{j+1}^{\dagger} \hat{c}_{j+1}\right\rangle \hat{f}_{j}\right.\right. \\
& \left.\left.+\left\langle\hat{c}_{j}^{\dagger} \hat{c}_{j}\right\rangle\left\langle\hat{c}_{j+1}^{\dagger} \hat{c}_{j+1}\right\rangle\right)\right\} .
\end{aligned}
$$

In the final line, we can use Eq. (3.11) to write the remaining fluctuation operators in terms of density operators again and afterwards replace the expectation values 
$\left\langle\hat{c}_{j}^{\dagger} \hat{c}_{j}\right\rangle$ with the guessed electron density $\rho(j)$ :

$$
\begin{aligned}
\hat{H} & \approx \sum_{j=1}^{N-1}\left\{-t\left(\hat{c}_{j}^{\dagger} \hat{c}_{j+1}+\hat{c}_{j+1}^{\dagger} \hat{c}_{j}\right)\right. \\
& \left.+V\left[\rho(j) \hat{c}_{j+1}^{\dagger} \hat{c}_{j+1}-\rho(j) \rho(j+1)+\rho(j+1) \hat{c}_{j}^{\dagger} \hat{c}_{j}\right]\right\} \\
& =\sum_{j=1}^{N-1}\left\{-t\left(\hat{c}_{j}^{\dagger} \hat{c}_{j+1}+\hat{c}_{j+1}^{\dagger} \hat{c}_{j}\right)\right. \\
& \left.\left.+V[\rho(j-1)+\rho(j+1)] \hat{c}_{j}^{\dagger} \hat{c}_{j}-2 V \rho(j) \rho(j+1)\right)\right\} .
\end{aligned}
$$

The final term in this Hamiltonian is a constant that can be removed by a suitable redefinition of the zero of energy (which is always arbitrary). Since the charge density is a smooth and continuous function, we can also use the approximation

$$
\left\langle\hat{c}_{j-1}^{\dagger} \hat{c}_{j-1}+\hat{c}_{j+1}^{\dagger} \hat{c}_{j+1}\right\rangle \approx 2 \rho(j)
$$

for the final form of the mean-field CDW Hamiltonian:

$$
\hat{H} \approx \sum_{j=1}^{N-1}\left\{-t\left(\hat{c}_{j}^{\dagger} \hat{c}_{j+1}+\hat{c}_{j+1}^{\dagger} \hat{c}_{j}\right)+2 V \rho(j) \hat{c}_{j}^{\dagger} \hat{c}_{j}\right\}=\hat{H}_{\mathrm{MF}} .
$$

In this mean-field Hamiltonian, we can now explicitly write for the electron density [103]:

$$
\rho(j)=A \cos (Q j a+\phi) .
$$

Finding and minimizing the ground state energy, in principle allows us to check that this guess is self-consistent. Since it is not essential for the CDW topology, we will not elaborate on the self-consistency conditions here.

\section{B The Fourier-transformed Hamiltonian}

Assuming periodic boundary conditions again, we can introduce creation and annihilation operators for electrons whose wave functions are the plane waves:

$$
\begin{aligned}
& \hat{c}_{k}^{\dagger}=\sqrt{1 / N} \sum_{j=1}^{N} e^{-i k j a} \hat{c}_{j}^{\dagger}, \\
& \hat{c}_{k}=\sqrt{1 / N} \sum_{j=1}^{N} e^{i k j a} \hat{c}_{j} .
\end{aligned}
$$


Notice that the plane-wave electrons can be interpreted as a (discrete) Fourier transformation of the localized electrons. Because of the assumed periodicity of the chain in real space, the plane wave amplitude at position $j$ has to be equal to that on position $j+N$. For the $1 D$ chain then, $k$ can be allowed to have discrete values $2 \pi m /(N a)$, with $m \in\{1,2, \ldots, N\}$. Moreover because the chain consists of discrete atoms, and the electronic wave function can only have a nonzero value at atomic positions, the wave numbers turn out to be periodic. This means that $\hat{c}_{k=2 \pi / a}^{\dagger}$ creates the same electronic wave function (that is, it has the same amplitude on each of the discrete atomic sites) as $\hat{c}_{k=0}^{\dagger}$.

We can also express the localized electrons in terms of the plane-wave ones:

$$
\begin{aligned}
& \hat{c}_{j}^{\dagger}=\sqrt{1 / N} \sum_{0 \leq k<2 \pi / a} e^{i k j a} \hat{c}_{k}^{\dagger}, \\
& \hat{c}_{j}=\sqrt{1 / N} \sum_{0 \leq k<2 \pi / a} e^{-i k j a} \hat{c}_{k} .
\end{aligned}
$$

These equations can be substituted directly into the Hamiltonian of Eq. (3.16):

$$
\begin{aligned}
\hat{H}_{\mathrm{MF}}= & \frac{1}{N} \sum_{j} \sum_{k} \sum_{k^{\prime}}\left[-t e^{i k j a} e^{-i k^{\prime}(j+1) a}-t e^{i k(j+1) a} e^{-i k^{\prime} j a}\right. \\
& \left.+2 V A \cos (Q j a+\phi) e^{i k j a} e^{-i k^{\prime} j a}\right] \hat{c}_{k}^{\dagger} \hat{c}_{k^{\prime}} .
\end{aligned}
$$

In these expressions, we can write the cosine as a sum of exponents:

$$
2 \cos (Q j a+\phi)=e^{i(Q j a+\phi)}+e^{-i(Q j a+\phi)} .
$$

Applying the definition of the Dirac delta function

$$
\delta_{k, k^{\prime}}=(1 / N) \sum_{j} e^{i\left(k-k^{\prime}\right) j a}
$$

then allows us to perform the sum over one of the momenta:

$$
\begin{aligned}
\hat{H}_{\mathrm{MF}} & =\frac{1}{N} \sum_{k} \sum_{k^{\prime}}\left(-t \delta_{k, k^{\prime}} e^{-i k^{\prime} a}-t \delta_{k, k^{\prime}} e^{i k a}\right. \\
& \left.+V A e^{i \phi} \delta_{k+Q, k^{\prime}}+V A e^{-i \phi} \delta_{k-Q, k^{\prime}}\right) \hat{c}_{k}^{\dagger} \hat{c}_{k^{\prime}} \\
& =\sum_{0 \leq k<2 \pi / a}\left\{-2 t \cos (k a) \hat{c}_{k}^{\dagger} \hat{c}_{k}+V A e^{i \phi} \hat{c}_{k}^{\dagger} \hat{c}_{k+Q}+V A e^{-i \phi} \hat{c}_{k}^{\dagger} \hat{c}_{k-Q}\right\}
\end{aligned}
$$




\section{C The Hamiltonian in matrix form}

To facilitate the use of numerical software for calculating the eigenvalues of the Hamiltonian, it is convenient to write it in matrix form:

$$
\hat{H}_{\mathrm{MF}}=\sum_{k}\left(\hat{c}_{k+Q}^{\dagger}, \hat{c}_{k+2 Q}^{\dagger} \ldots \hat{c}_{k+q Q}^{\dagger}\right) H_{k}\left(\begin{array}{c}
\hat{c}_{k+Q} \\
\hat{c}_{k+2 Q} \\
\vdots \\
\hat{c}_{k+q Q}
\end{array}\right) .
$$

In this expression, we assumed $Q=n 2 \pi / a$, with $n=p / q$ a co-prime fraction. While writing the Hamiltonian this way, however, one should be careful with the sum over wave numbers. If we simply sum $k$ over the values $2 \pi m /(N a)$, with $m \in\{1,2, \ldots, N\}$, electrons with momentum equal to for example $3 Q / 2$ will be created both by the first component $\hat{c}_{k+Q}^{\dagger}$ of the vector of creation operators (for $k=Q / 2$ ), and by the final component $\hat{c}_{k+q Q}^{\dagger}$ in the vector (for $k=3 Q / 2$ ). The eigenvalues of the Hamiltonian matrix $H_{k}$, however, will correspond directly to the energies of $\hat{H}_{\mathrm{MF}}$ only if it is expressed in an orthonormal basis, or equivalently if the every possible electron state is created only once within the sum over $k$. This can be achieved by restricting the range of momentum values summed over to $2 \pi m /(N a)$, with $m \in\{1,2, \ldots, N / q\}$. Notice that the restricted range of momenta consists precisely of all momenta within the so-called reduced BZ that corresponds to the enlarged real-space unit cell of size $q a$ in the CDW state.

To find the matrix $H_{k}$ for the $1 D$ chain, we thus first rewrite the equation for $\hat{H}_{\mathrm{MF}}$ such that it contains only a sum over this restricted range of momentum values:

$$
\begin{aligned}
\hat{H}_{\mathrm{MF}} & =\sum_{0 \leq k<2 \pi / a}\left\{\epsilon_{k} \hat{c}_{k}^{\dagger} \hat{c}_{k}+V A e^{i \phi} \hat{c}_{k}^{\dagger} \hat{c}_{k+Q}+V A e^{-i \phi} \hat{c}_{k}^{\dagger} \hat{c}_{k-Q}\right\} \\
& =\sum_{0 \leq k<2 \pi / q a} \sum_{n=1}^{q}\left\{\epsilon_{k+n Q} \hat{c}_{k+n Q}^{\dagger} \hat{c}_{k+n Q}+V A e^{i \phi} \hat{c}_{k+n Q}^{\dagger} \hat{c}_{k+(n+1) Q}\right. \\
& \left.+V A e^{-i \phi} \hat{c}_{k+(n+1) Q}^{\dagger} \hat{c}_{k+n Q}\right\} .
\end{aligned}
$$

In this expression, we used $(q+1) Q=Q$, which follows from the periodicity of the wave numbers.

Writing out this equation in the desired matrix form, yields the final expression 
3 Visualizing topological transport in charge-ordered materials

for $H_{k}$ :

$$
H_{k}=\left(\begin{array}{cccccc}
\epsilon_{k+Q} & \tilde{\Delta} & 0 & \ldots & 0 & \tilde{\Delta}^{*} \\
\tilde{\Delta}^{*} & \epsilon_{k+2 Q} & \tilde{\Delta} & 0 & \ldots & 0 \\
0 & \tilde{\Delta}^{*} & \ddots & \ddots & & \vdots \\
\vdots & 0 & \ddots & & & 0 \\
0 & \vdots & & & & \tilde{\Delta} \\
\tilde{\Delta} & 0 & \ldots & 0 & \tilde{\Delta}^{*} & \epsilon_{k+q Q}
\end{array}\right)
$$

Recall that here, $\tilde{\Delta}=V A e^{i \phi}$, and $k+q Q=k$ owing to the periodicity of the wave numbers. Note that this form of the matrix does not apply to the special case $q=2$, which we do not consider.

\section{D Calculating the topological invariant}

To clarify how the Chern number can be straightforwardly calculated for chargeordered materials, we consider a $1 / 3$ filled chain with periodic boundary conditions. The Hamiltonian $H_{k}$ from Eq. (3.8) then reads

$$
H_{k}(k)=\left[\begin{array}{ccc}
-2 t \cos k a & V A e^{i \phi} & V A e^{-i \phi} \\
V A e^{-i \phi} & -2 t \cos (k a+2 \pi / 3) & V A e^{i \phi} \\
V A e^{i \phi} & V A e^{-i \phi} & -2 t \cos (k a+4 \pi / 3)
\end{array}\right]
$$

The amplitude of the mean-field parameter $V A$ may be assumed to be much smaller than $t$. The $1 / 3$ filling implies that the chemical potential lies within the lowest energy gap for all values of $\phi$. The periodic boundary conditions remove all edge states from the spectrum and allows the Chern number to be calculated as an integral over the periodic BZ.

This Hamiltonian has the same form as Harper's equation, describing the IQHE, upon identifying $k \rightarrow k_{x}$ and $\phi \rightarrow k_{y}$. Its energy spectrum is presented in Fig. 3.1a.

The Chern number $c_{m}$ may now be computed as an integral of the Berry curvature $\Omega_{k \phi}^{m}$ over the first BZ, calculated for the one occupied band of energy eigenstates:

$$
\begin{aligned}
\Omega_{k \phi}^{m} & =\left\langle\partial_{k} \psi_{m} \mid \partial_{\phi} \psi_{m}\right\rangle-\left\langle\partial_{\phi} \psi_{m} \mid \partial_{k} \psi_{m}\right\rangle, \\
c_{m} & =\frac{1}{2 \pi i} \int_{0}^{2 \pi} d \phi \int_{B Z} \Omega_{k \phi}^{m} d k .
\end{aligned}
$$

The resulting Chern number is the topological index, which characterizes the 
CDW and yields the number of electrons in the $m^{t h}$ band that are adiabatically transported around the chain upon varying the phase $\phi$ over one period.

Because the Chern number is a topological quantity, it is invariant under transformations of the Hamiltonian that affect both its eigenvalues and eigenvectors, as long as the transformations do not cause any band inversions. We use this fact to calculate the Chern number for the CDW in a straightforward manner. All that is needed is to consider the three special points $k_{1}=-\pi / 3 a, k_{2}=0$ and $k_{3}=\pi / 3 a$, at which band inversions might occur. At these points, the eigenstates can be approximated by leaving all off-diagonal elements out of the Hamiltonian, except for those two that connect the two states which are nearly degenerate. Since $1 / 3$ filling involves only the lowest band, we are interested only in the near degeneracies at $k_{1}$ and $k_{3}$. Near the point $k_{1}$, the lowest energy eigenstate can then be approximated by writing

$$
\begin{gathered}
H_{k}\left(k_{1}, \phi\right) \approx\left[\begin{array}{ccc}
-t & V A e^{i \phi} & 0 \\
V A e^{-i \phi} & -t & 0 \\
0 & 0 & t
\end{array}\right], \\
\left|\psi_{1}\right\rangle \equiv\left|\psi\left(k_{1}, \phi\right)\right\rangle=\frac{1}{\sqrt{2}}\left(\begin{array}{c}
1 \\
-e^{-i \phi} \\
0
\end{array}\right) .
\end{gathered}
$$

For point $k_{3}$, we should instead consider

$$
\begin{gathered}
H_{k}\left(k_{3}, \phi\right) \approx\left[\begin{array}{ccc}
-t & 0 & V A e^{-i \phi} \\
0 & 2 t & 0 \\
V A e^{i \phi} & 0 & -t
\end{array}\right], \\
\left|\psi_{2}\right\rangle \equiv\left|\psi\left(k_{3}, \phi\right)\right\rangle=\frac{1}{\sqrt{2}}\left(\begin{array}{c}
1 \\
0 \\
-e^{i \phi}
\end{array}\right) .
\end{gathered}
$$

In between these two near degeneracies, at $k=0$, the lowest energy state is far from all other states, and may be approximated as $\left|\psi\left(k_{2}, \phi\right)\right\rangle=(1,0,0)^{\dagger}$.

The eigenvectors in Eq. (3.34) and Eq. (3.36) are determined up to an arbitrary phase factor only. The calculation of the Chern number will become particularly straightforward if we divide the BZ into two parts, $S_{1} \in\left[k_{1}, k_{2}\right]$ and $S_{2} \in\left[k_{2}, k_{3}\right]$, and choose different conventions for the phase of the eigenvectors in each region. The lowest energy eigenstates can then be defined to be:

$$
\left|\Psi_{1}(k, \phi)\right\rangle=\frac{1}{\sqrt{2}}\left(\begin{array}{c}
\sqrt{2-\alpha^{2}(k)} \\
\alpha(k) e^{-i \phi} \\
0
\end{array}\right)
$$


3 Visualizing topological transport in charge-ordered materials

$$
\left|\Psi_{2}(k, \phi)\right\rangle=\frac{1}{\sqrt{2}}\left(\begin{array}{c}
\sqrt{2-\beta^{2}(k)} \\
0 \\
-\beta(k) e^{i \phi}
\end{array}\right) .
$$

Here, $\alpha(k)$ is some real function with values 1 and 0 at momenta $k_{1}$ and $k_{2}$ respectively, and $\beta(k)$ is a real function with values 0 and 1 at momenta $k_{2}$ and $k_{3}$ respectively. The formula for the Berry curvature given in Eq. (3.32) then yields

$$
S_{1}: \Omega_{k \phi}^{1}=-i \alpha^{\prime} \alpha, \quad S_{2}: \Omega_{k \phi}^{1}=i \beta \beta^{\prime} .
$$

The Chern number can be found to be

$$
c_{1}=\frac{1}{2 \pi i} \int_{0}^{2 \pi} d \phi\left[-i \int_{k_{1}}^{k_{2}} \alpha^{\prime} \alpha d k+i \int_{k_{2}}^{k_{3}} \beta \beta^{\prime} d k\right]=+1 .
$$

This result shows that the CDW acts as a Thouless pump, by transferring one electron through the wire connecting its ends as the charge order slides by one wavelength. This is the CDW-analogue of the well-known result for the IQHE [80].

A similar calculation will show that the Chern number $c_{2}$ for the second band equals -2 while that for the uppermost band is again 1. In terms of quantized transport between edges in an open CDW chain, this means that for filling fraction $n=1 / 3$ sliding the CDW over a single wave length results in a single electron being transferred in the direction of sliding. For a filling of $n=2 / 3$ we have $c_{1}+c_{2}=-1$, so that a single electron will instead be transferred in the direction opposite to the sliding motion. 


\section{Introduction to solitary waves}

\subsection{Introduction}

Solitary waves were introduced in physics by J.S. Russell in 1834 [121], after he observed one traveling for miles along the Union Canal near Edinburgh, Scotland, without altering its shape or speed. The dynamics of this particular wave was later described using the Korteweg-de Vries equation [122], but the idea of solitary waves as stable, localized configurations with finite energy in any medium or field [123] turned out to be much more general. They are now known to occur and play an important role in almost all areas of physics, including particle physics [124], cosmology [125, 126], (nonlinear) optics [127, 128], condensed-matter theory [129131], and biophysics [132].

To understand the generic properties of solitary waves, one can consider in the most elementary models or field theories possible. Two famous examples are the sine-Gordon and the $\varphi^{4}$ models.

The sine-Gordon model is an integrable model [133] with infinitely many conserved quantities in its field configurations. The solitary waves in this model can pass through one another while retaining their individual sizes and shapes [134], and for this reason they are named solitons. Solitons have found many applications including, for example, the analysis of seismic data [135], convecting nematic fluids [136], Josephson-junction arrays [137, 138], and magnetic materials [139].

The $\varphi^{4}$ theory was first introduced by Ginzburg and Landau as a phenomenological theory of second-order phase transitions [25]. Since then, it has been identified as a low-energy effective description of phenomena in almost any field of physics, making the detailed understanding of its fundamental properties and excitations particularly relevant. The $\varphi^{4}$ theory can be extended to higher order [68, 140-142], as well as more structured fields [143-148], but the classical scalar theory already contains all essential ingredients required to describe the emergence, dynamics, and 


\section{Introduction to solitary waves}

interactions of solitary waves. In contrast to the sine-Gordon model, the $\varphi^{4}$ theory is not-integrable, and although it possesses stable and localized solitary wave excitations of finite energy called kinks [123], they cannot pass through one another unaffected, as the solitons do in the sine-Gordon model [59].

\subsection{Topological sectors}

In this section, we review the main general concepts of classical field theories. The reader familiar with these concepts might wish to skip it.

Consider a classical, real, and scalar field $\varphi=\varphi(t, x)$ in $(1+1)$-dimensional space-time $[59,149,150]$. Its dynamics is determined by the Lagrangian density:

$$
\mathcal{L}=\frac{1}{2}\left(\frac{\partial \varphi}{\partial t}\right)^{2}-\frac{1}{2}\left(\frac{\partial \varphi}{\partial x}\right)^{2}-U(\varphi)
$$

The specific potential $U(\varphi)=m^{2} \varphi^{2}$ yields a free scalar field theory, whose equation of motion is described by the classical Klein-Gordon equation. More generally, the function $U(\varphi)$ can be thought of as a self-interaction potential of the field $\varphi$. We can always use the freedom to choose the zero of energy to ensure that $U(\varphi)$ is a nonnegative function of $\varphi$, whose minimum value is precisely zero.

Using the Euler-Lagrange equation, the equation of motion for $\varphi(t, x)$ is found to be:

$$
\frac{\partial^{2} \varphi}{\partial t^{2}}-\frac{\partial^{2} \varphi}{\partial x^{2}}+\frac{d U}{d \varphi}=0
$$

For a static, time independent solution this simplifies to $d^{2} \varphi / d x^{2}=d U / d \varphi$. The dynamics of an initial field configuration $\varphi\left(t_{0}, x\right)$ may be studied by numerically solving the equation of motion on a discrete lattice [61, 151].

The energy of any field configuration $\varphi(x, t)$ is given by the functional:

$$
E[\varphi]=\int_{-\infty}^{+\infty}\left[\frac{1}{2}\left(\frac{\partial \varphi}{\partial t}\right)^{2}+\frac{1}{2}\left(\frac{\partial \varphi}{\partial x}\right)^{2}+U(\varphi)\right] d x
$$

Notice that in spite of the time dependence of $\varphi$, the energy $E[\varphi]$ is a timeindependent, conserved quantity. The energy of a static ground state field configuration is sometimes referred to as the mass of that field. This should not be confused with the Klein-Gordon mass parameter $m$ in a free field theory. For the energy in Eq. (4.3) to be finite, the integral should converge. This yields the requirement that all physical fields $\varphi(t, x)$ approach a minimum of $U(\varphi)$ sufficiently quickly as $x$ approaches positive or negative infinity. 
If there is only a single minimum in $U(\varphi)$, at $\varphi=\varphi_{v}^{(1)}$, then the field configuration $\varphi(t, x)=\varphi_{v}^{(1)}$ is the vacuum or ground state of the system. If there are multiple, degenerate minima $\varphi_{v}^{(1)}, \varphi_{v}^{(2)}$, and so on, they together form a vacuum manifold and any field configuration $\varphi(t, x)=\varphi_{v}^{(j)}$ is a possible ground state. It is also feasible however, to find static field configurations that approach distinct minima at opposing boundaries of space $(x= \pm \infty)$. These types of solutions are called topological [149, 150, 152], and more generally, one may divide all static configurations into topological sectors labelled by the set of minima they approach at spatial infinity. This is indicated schematically in Fig. 4.1.

The energy of a static field in any topological sector may be written in a particularly convenient form by introducing the so-called superpotential $W(\varphi)$, defined by:

$$
U(\varphi)=\frac{1}{2}\left(\frac{d W(\varphi)}{d \varphi}\right)^{2}
$$

The superpotential always exists for the nonnegative function $U(\varphi)$, which is our key assumption. Using the superpotential, the expression for the energy in Eq. (4.3) can be written for a static field configuration as:

$$
\begin{aligned}
E[\varphi] & =\frac{1}{2} \int_{-\infty}^{+\infty}\left[\left(\frac{d \varphi}{d x}\right)^{2}+\left(\frac{d W}{d \varphi}\right)^{2}\right] d x=\frac{1}{2} \int\left(\frac{d \varphi}{d x}-\frac{d W}{d \varphi}\right)^{2} d x+\int \frac{d W}{d \varphi} \frac{d \varphi}{d x} d x \\
& =\frac{1}{2} \int\left(\frac{d \varphi}{d x}-\frac{d W}{d \varphi}\right)^{2} d x+\left(\left.W\right|_{\varphi(x=+\infty)}-\left.W\right|_{\varphi(x=-\infty)}\right) .
\end{aligned}
$$

From the final line, it is clear that any field in a given topological sector necessarily has an energy $E \geq E_{\mathrm{BPS}}$, with the minimum possible energy $E_{\mathrm{BPS}}=$ $\left(\left.W\right|_{\varphi(x=+\infty)}-\left.W\right|_{\varphi(x=-\infty)}\right)$. It always can be chosen to be positive, so it is regarded as a soliton mass and is named after Bogomolny, Prasad, and Sommerfield $[153,154]$, we will denote it by $M$. Any field configuration with energy equal to $E_{\mathrm{BPS}}$ is said to saturate the BPS bound.

Since the integral in the final line of Eq. (4.5) is over a squared function, the only way to obtain a BPS saturated configuration is to have a field obeying the condition:

$$
\frac{d \varphi}{d x}=\frac{d W}{d \varphi}=\sqrt{2 U} .
$$

If such a field configuration does exist, it is guaranteed by the variational principle to be a ground state for its topological sector. The Euler-Lagrange Eq. (4.2) is therefore automatically satisfied by solutions of Eq. (4.6), even though the latter is 
(a)

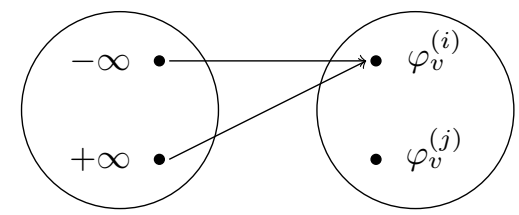

(c)

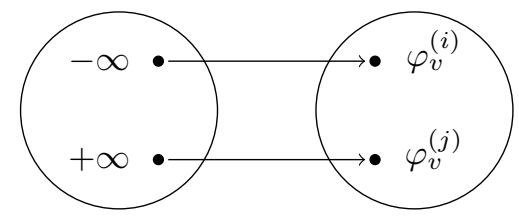

(b)

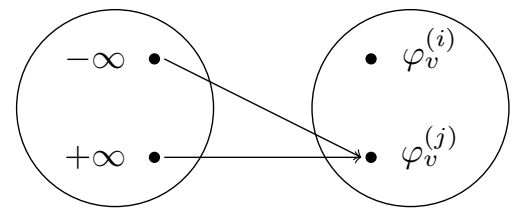

(d)

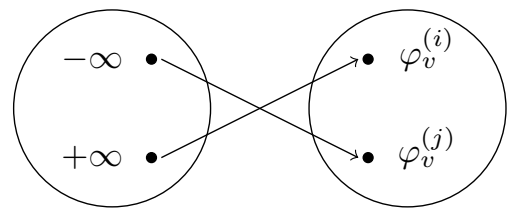

Figure 4.1: (a) - (b) Scheme of asymptotic behavior at $x \rightarrow \pm \infty$ of nontopological solutions, e.g. the vacuum, when $\varphi_{v}^{(i)}=\varphi_{v}^{(j)}$. (c) - (d) Scheme of asymptotic behavior at $x \rightarrow \pm \infty$ of topological solutions, when $\varphi_{v}^{(i)} \neq \varphi_{v}^{(j)}$.

only a first order differential equation.

\subsection{Solitons in the sine-Gordon model}

In this section, we review the main general concepts of the sine-Gordon model. The reader familiar with these concepts might wish to skip it.

The self-potential in Eq. (4.1) of the sine-Gordon model reads

$$
U(\varphi)=\frac{m^{2}}{\beta^{2}}(1-\cos \beta \varphi),
$$

but in the following we will use the dimensionless form

$$
U(\varphi)=1-\cos \varphi
$$

which is presented in Fig. 4.2a. This potential has a vacuum manifold $\varphi_{v}^{(j)}=2 \pi j$ for any integer $j$. The equation of motion Eq. (4.2) for this potential becomes:

$$
\frac{\partial^{2} \varphi}{\partial t^{2}}-\frac{\partial^{2} \varphi}{\partial x^{2}}+\sin \varphi=0
$$

A nontrivial topological static solution, i.e. a soliton is

$$
\varphi_{S}(x)=4 \arctan [\exp (x-a)]
$$


where $a$ is an arbitrary center of the soliton that is characterized by width $l_{S} \simeq 1$. The plot of this function in presented in Fig. 4.2b. This solution satisfies not only Eq. (4.9), but also Eq. (4.6) for potential in Eq. (4.8). It means that it is a stable configuration, with the minimum possible energy $M_{S}=8$ from Eq. (4.5) for any field connecting two distinct vacua at zero and $2 \pi$. Another topological solution, called antisoliton and written $\varphi_{\bar{S}}=-\varphi_{S}$, connects the same two vacua, but in the opposite direction.

Finally, owing to the Lorentz invariance of the d'Alembert operator in Eq. (4.9), the static (anti)soliton in Eq. (4.10) may be boosted to yield a dynamical one, that moves with a constant velocity $v$ :

$$
\varphi_{S}(t, x)= \pm 4 \arctan \left[\exp \left(\frac{x+a-v t}{\sqrt{1-v^{2}}}\right)\right] .
$$

Here, $v$ is measured in units of the speed of light with respect to the choice of units in Eq. (4.9), where speed of light is equal to one in the d'Alembert operator.

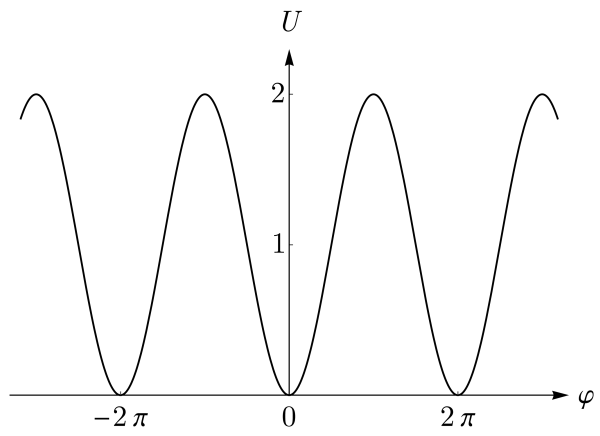

(a)

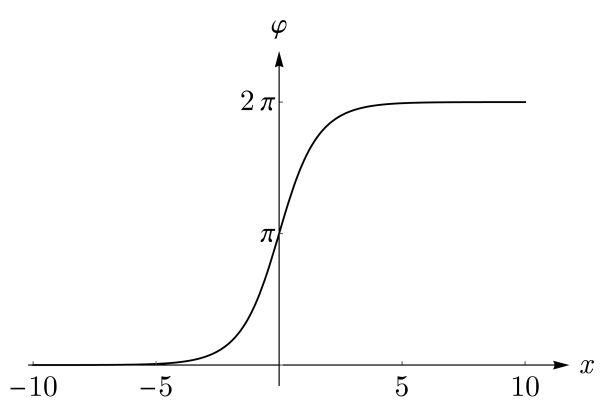

(b)

Figure 4.2: (a) The self-interaction potential $U$ for the sine-Gordon model given by Eq. (4.8). (b) The soliton solution for the sine-Gordon model for $a=0$ given by Eq. (4.10).

An interesting feature of this model is that one can find analytically one more solution called breather [155], a localized nontopological infinitely-long-lived solution that is periodic in time. "Like" this breather, a quasi-long-lived solution in the $\varphi^{4}$ model was found (or bion in $(1+1)$ dimensions, it will be discussed later in Section 6.4 of Chapter 6) that is called quasi-breather [64]. However, there is a big difference between these two solutions. In the sine-Gordon model, it arises as an independent solution of the equation of motion, while in the $\varphi^{4}$ theory it is a consequence of interactions between two solitary waves. The analytical studies of the breathers with perturbation theory converged in the case of the sine-Gordon 


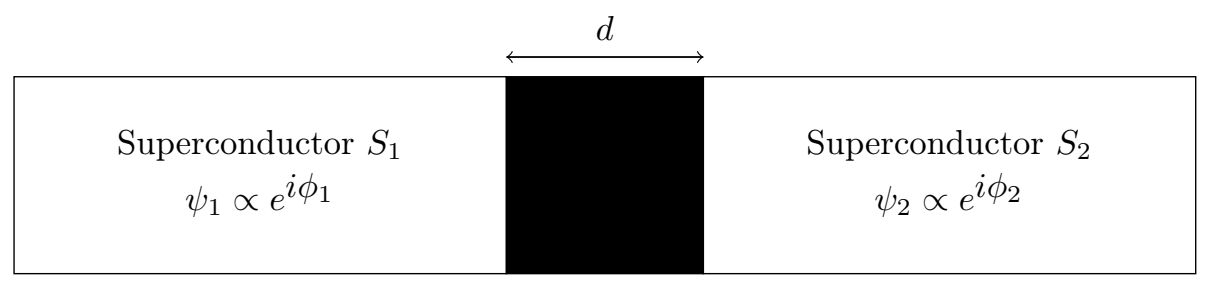

Insulator

Figure 4.3: The Josephson junction.

theory, while for the $\varphi^{4}$ model it was only asymptotic. This means that to some order in perturbation theory the spatial localization of the breather is destroyed. Hence, that only solutions with a small amplitude of oscillating tails extending to infinity and of infinite energy are possible in the continuum theory [156]. The names breather for the sine-Gordon model and quasi-breather for the $\varphi^{4}$ model have been chosen historically due to visual similarity, and are still used, although the nature of these two objects is completely different.

\subsection{Josephson effect}

As we mentioned above the sine-Gordon model has a lot of applications in physics. Now, we dwell one such application in detail. The Josephson effect $[157,158]$ is a phenomenon characterized by an electric current that flows indefinitely without any applied voltage, across two superconductor regions $S_{1}$ and $S_{2}$ (see Fig. 4.3) separated by a few nanometer layer of an insulating material (width $d$ ) that acts as a potential barrier. This device is called a Josephson junction because the physical phenomenon was theoretically predicted by Brian David Josephson [20] in 1962 (Nobel prize in 1973). It was then experimentally verified a year after [21].

Originally, the wavefunctions $\psi_{1}$ and $\psi_{2}$ and the phases $\phi_{1}$ and $\phi_{2}$ of the two superconductors $S_{1}$ and $S_{2}$ are independent (see Fig. 4.3). By decreasing the insulating interlayer one can experimentally measure the electromagnetic radiation from the point contact with the frequency $\nu=V K_{J}$, where $V$ is an applied transition voltage, $K_{J}=\Phi_{0}^{-1}$ is the Josephson constant, and $\Phi_{0}=h / 2 e$ is the magnetic flux quantum. Note that in Chapter 2 we already faced a magnetic flux quantum in the IQHE $\phi_{0}=h / e$. The differences in factors may be explained by the number of quantized electron-carriers. In this state, the system may be described by a single macroscopic wave function $\psi \propto \exp (i \phi)$. That means that electrons are going through the Josephson contact (Josephson tunneling) like so-called Cooper pairs (becoming one boson instead of two fermions), and here $\phi$ is the quantum phase 
common to all Cooper pairs. This phase has a shape of the soliton, however, it is not yet related to the sine-Gordon model, because $V$ is fixed and the "soliton" cannot move in this system. The interesting physical quantity in this experiment is a quantum of magnetic flux $\Phi_{0}$, or a fluxon, that has a soliton-like behavior [158, 159]. It corresponds to the difference $\pm 2 \pi$ in the quantum phase $\phi$ in the wave function $\psi$ across the insulator separating the two superconducting electrodes. To obtain it one should consider a ring geometry and a magnetic field passing through it. This yields a static soliton because the position of the insulator is fixed. To obtain a moving soliton, one has to consider a Josephson junction array, namely an array of rings. Each ring then is characterized by its own phase, and this phase difference can move in between rings representing the soliton motion.

The above mentioned soliton is described by some modification of Eq. (4.9) [159], and the Josephson effect allows to observe the soliton dynamics in a real setup. For example, single-soliton [160] and multi-soliton [161, 162] dynamics were studied in a ring-shaped junction geometry. Using low-temperature scanning electron microscopy, one may also consider the Lorentz contraction of soliton [163] and multi-soliton modes in long junctions [164-167].

Besides nonlinear solitons, the sine-Gordon equation given by Eq. (4.9) has another solution $\varphi \propto \exp [i(k x-\omega t)]$ in linear order approximation $(\sin \varphi \simeq \varphi)$, which is called plasma waves, with dispersion relation $\omega^{2}=1+k^{2}$, where $k$ is some wavenumber and $\omega$ is some frequency. In real setups, plasma waves are generated when fluxons move through some inhomogeneity in the junction [24], which might influence the motion of fluxons.

In most real samples, one cannot avoid the presence of inhomogeneities and defects. Thus, it is interesting to study the influence of an inhomogeneity on the soliton dynamics in the sine-Gordon model. Some researches already looked at this model in presence of perturbation [39, 168, 169], including the variable mass the sine-Gordon model [170-172]. We will consider another type of inhomogeneity. Because it is always interesting to compare the effects of integrability and nonintegrability, we study both, the sine-Gordon and the $\varphi^{4}$ models simultaneously.

\subsection{Kinks in the $\varphi^{4}$ model}

In this section, we review the main general concepts of the $\varphi^{4}$ model. The reader familiar with these concepts might wish to skip it.

The simplest scalar field theory having distinct topological sectors is the so-called the $\varphi^{4}$ theory. It is defined as a particular instance of the general model of Eq. (4.1), with the self-interaction potential equal to

$$
U(\varphi)=\frac{\lambda}{4}\left(\frac{m^{2}}{\lambda}-\varphi^{2}\right)^{2}
$$




\section{Introduction to solitary waves}

and in the dimensionless form

$$
U(\varphi)=\frac{1}{4}\left(1-\varphi^{2}\right)^{2},
$$

which is presented in Fig. 4.4a. The equation of motion given by Eq. (4.2) with this potential becomes

$$
\frac{\partial^{2} \varphi}{\partial t^{2}}-\frac{\partial^{2} \varphi}{\partial x^{2}}+\varphi^{3}-\varphi=0
$$

This equation has a finite number of trivial solutions, in contrast to Eq. (4.9), given by $\varphi=0, \varphi_{v}^{(1)}=-1$, and $\varphi_{v}^{(2)}=1$. The solution $\varphi=0$, sitting at a maximum of Eq. (4.13), is unstable, while the solutions $\varphi_{v}= \pm 1$ form two stable and degenerate ground states.

The nontrivial solutions of Eq. (4.14) lie in a topological sector where the field approaches one minimum as $x \rightarrow-\infty$, and the other minimum as $x \rightarrow \infty$. One such stable configuration, with the minimum possible energy for this choice of two distinct vacua is

$$
\varphi_{K}(x)=\tanh \left(\frac{x-a}{\sqrt{2}}\right),
$$

where $a$ is a center of the kink. It is easily checked that this solution satisfies both Eq. (4.14), and the BPS condition of Eq. (4.6) with substituted Eq. (4.13). This topological solution is often called a kink, and it is presented in Fig. 4.4b. The center of the kink lies at the position $x=a$, and it has a characteristic width $l_{K} \simeq \sqrt{2}$. Another topological solution, called antikink, and written $\varphi_{\bar{K}}=-\varphi_{K}$, connects the same two vacua, but in the opposite direction.

Because the energy does not depend on the kink position $a$, translations of the kink in space may be interpreted as zero-energy excitations. There is also a stable excitation mode of the kink at nonzero energy [150], which can be interpreted as an internal or vibrational mode (see Appendix 4.A).

From Eq. (4.5) the value $M_{K}=2 \sqrt{2} / 3$ can be found for the energy or mass of the kink. Finally, owing to the Lorentz invariance of Eq. (4.14), the static kink solution may be boosted to yield a dynamical solution in which the kink (or antikink) moves with a constant velocity

$$
\varphi_{K}(t, x)= \pm \tanh \left(\frac{x+a-v t}{\sqrt{2\left(1-v^{2}\right)}}\right) .
$$

Here, $v$ is the velocity of the kink measured in units of the speed of light.

Apart from trivial solutions and the kink the Eq. (4.14) has one more, that is 


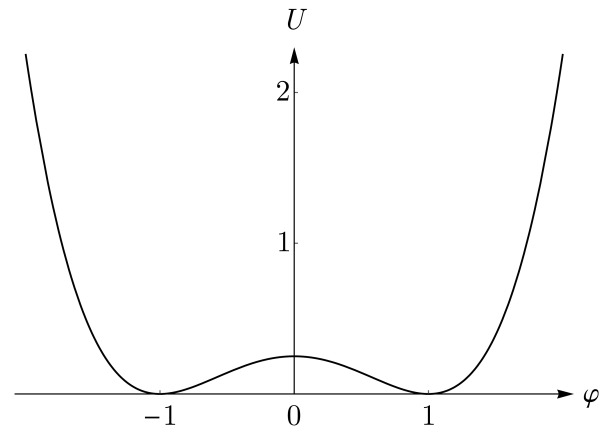

(a)

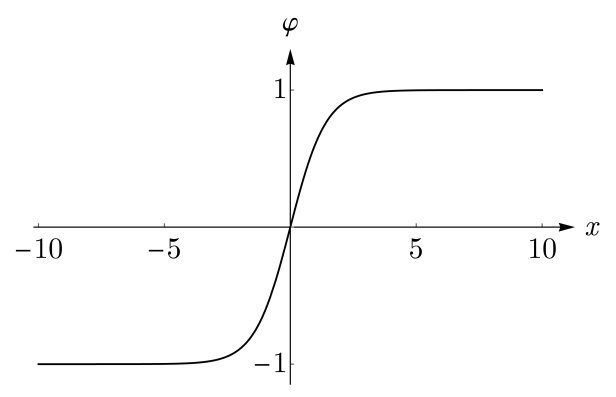

(b)

Figure 4.4: (a) The self-interaction potential $U$ for the $\varphi^{4}$ model given by Eq. (4.13). (b) The kink solution for the $\varphi^{4}$ model for $a=0$ given by Eq. (4.15).

periodic and may be expressed in terms of elliptic sine:

$$
\varphi(x)=\varphi_{0} \operatorname{sn}(b x, k), \quad \text { with } k^{2}=\frac{\varphi_{0}^{2}}{2-\varphi_{0}^{2}}, \quad b^{2}=1-\frac{\varphi_{0}^{2}}{2} .
$$

Here, the amplitude $\varphi_{0}$ is taken to lie between zero and one. In the limit $\varphi_{0} \rightarrow 1$, the elliptic sine solution approaches the kink configuration (see Fig. 4.5). For more detail see Appendix 4.B.

\subsection{Proteins}

One of the most exciting applications of the kinks of $\varphi^{4}$ model is an approach in the description of the tertiary structure of proteins [40-42]. The latter are polymers of amino acids, originally a one-dimensional chain (the primary structure), however, they usually may perform specific functions in cells only after correct folding in three dimensions (the tertiary structure). In [40] it was shown that the tertiary structure of proteins (to be more precise helix-loop-helix motif) may be successfully modeled resorting to an effective $\varphi^{4}$ field theory that is based on a local symmetry of proteins. In this approach, the geometrical configuration of a protein chain is represented by a one-dimensional curve with topologically stable inflection points, in which the curve becomes flat. These points are represented exactly by the kinks.

As an example, we reproduce here the results from [41], in which 153 amino-acid Myoglobin with Protein Data Bank (PDB) code 1M6C was reproduced using the above mentioned approximation. This particular backbone structure is known to a 


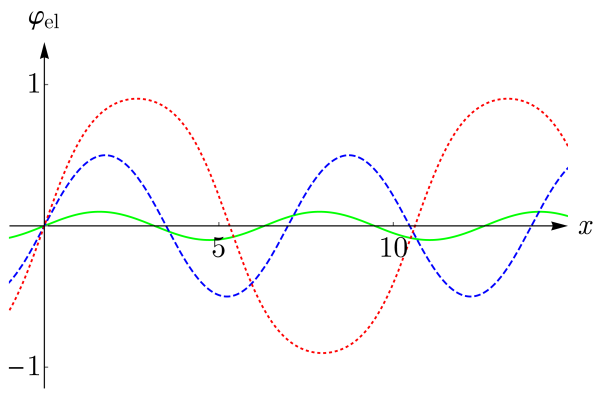

(a)

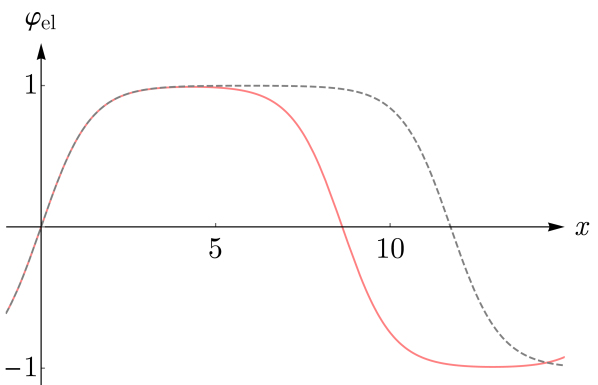

(b)

Figure 4.5: The solution $\varphi_{\mathrm{el}}(x)$ depending on the parameter $\varphi_{0}$. (a) For $\varphi_{0}=0.1$ (solid green line), $\varphi_{0}=0.5$ (dashed blue line) and $\varphi_{0}=0.9$ (dotted red line). (b) For $\varphi_{0}=0.991$ (solid pink line) and $\varphi_{0}=0.999$ (dashed gray line). The period of the plotted function expanses with the increasing of the value $\varphi_{0}$.

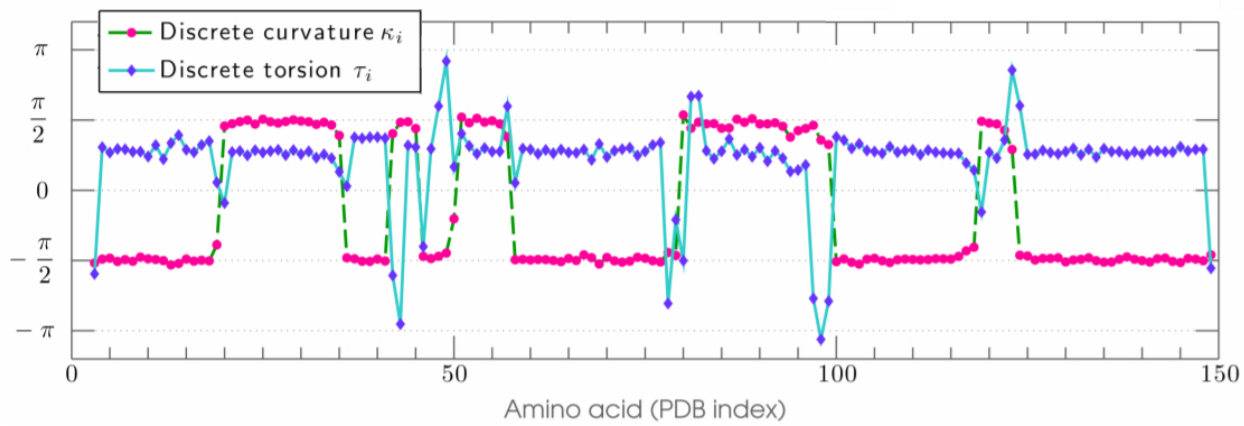

Figure 4.6: Visualisation of the protein structure by depicting torsion $\tau_{i}$ (blue dots) and bending $\kappa_{i}$ (pink dots) angels, where $\kappa_{i}$ changes a sign whenever there is an inflection point. The indexing refers to the position of amino acids along the backbone. Reprinted from [41].

resolution of $1.90 \AA$ in root-mean-square distance (RMSD) from X-ray crystallography [174]. In [41] was shown that entire fold can be encoded into some global parameters of the function that is introduced as an ansatz (similar [41] to Eq. (4.16))

$$
\tilde{x}(t)=\frac{b \exp \left[c_{1}\left(t-t_{0}\right)\right]-a \exp \left[c_{2}\left(t-t_{0}\right)\right]}{\exp \left[c_{1}\left(t-t_{0}\right)\right]+\exp \left[c_{2}\left(t-t_{0}\right)\right]}
$$




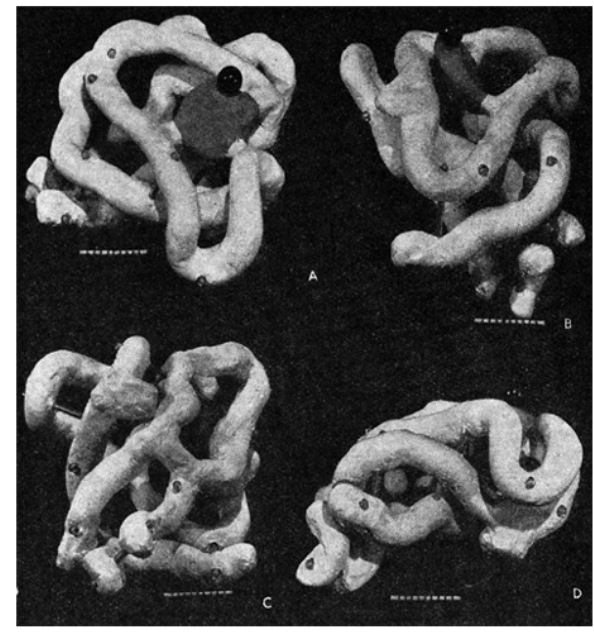

(a)

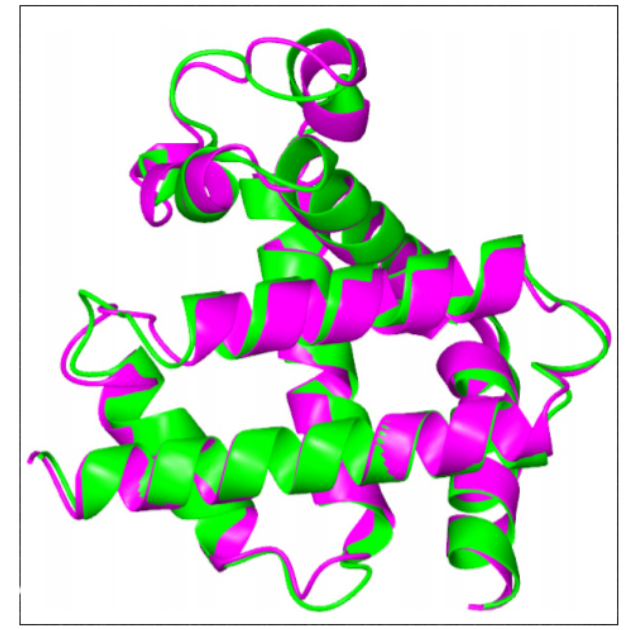

(b)

Figure 4.7: (a) Photographs of a model of the Myoglobin molecule. Polypeptide chains are white, the gray disk is the hem group. The spheres show positions at which heavy atoms were attached to the molecule. The marks on the scale are $1 \AA$. Reprinted from [173]. (b) The juxtaposition of the native three-dimensional structure of the $1 \mathrm{M} 6 \mathrm{C}$ protein from PDB (green) together with its reconstruction in terms of approximate ansatz (purple). The RMSD distance between two configurations is around $1.27 \AA$. Reprinted from [41].

that describes the geometry of a native folded protein. Here, $t$ is a length parameter that measures the distance along a static protein backbone and other parameters may be specified using a particular protein. As it was shown, a modification of this ansatz may describe bond $\kappa_{i}$ and torsion $\tau_{i}$ angles, and it has an atomic-level accuracy. In Fig. 4.6 a geometric structure of the $1 \mathrm{M} 6 \mathrm{C}$ protein is presented. In Fig. 4.7a we show X-ray crystallography and in Fig. 4.7b the juxtaposition of the native three-dimensional structure of the $1 \mathrm{M} 6 \mathrm{C}$ protein from PDB together with its reconstruction in terms of an approximate ansatz.

Today, modeling the protein structure that is based on classical molecular dynamics is promising, however, it is restricted only to some types of folding proteins [175]. For a more precise description of the tertiary structures much larger scale computing power than is available nowadays is necessary [176]. As a result, the gap between the number of known sequences and studied structures remains high. 


\section{Introduction to solitary waves}

On the contrary, protein dynamics within a Monte Carlo simulation of the field theory looks promising and shows that the protein clusters provide a mechanism of the protein folding [42] that is related to the functions of the proteins. According to the fact that the dynamics of the protein may be defined by the corresponding kink dynamics [42], we expect that our research of kink-impurity interactions might help in a better understanding kink dynamics, thus, make a step forward in the understanding of protein misfolding, therefore, the understanding of disease mechanisms. 


\section{Appendix}

\section{A Internal kink excitation mode}

To describe internal excitations of any static configuration $\varphi_{s}(x)$, we can consider small perturbations on top of the static solution [150]:

$$
\varphi(t, x)=\varphi_{s}(x)+\delta \varphi(t, x) .
$$

Here, $\varphi_{s}$ is a time-independent field satisfying the general Eq. (4.2). The perturbation $\delta \varphi(t, x)$ may have any shape, but we assume its amplitude $|\delta \varphi| \ll\left|\varphi_{s}\right|$ to be small compared to that of the static field.

Substituting this equation for the field $\varphi(t, x)$ into the Eq. (4.2), and keeping only terms up to linear order in the perturbation $\delta \varphi(t, x)$, yields the condition:

$$
\frac{\partial^{2} \delta \varphi}{\partial t^{2}}-\frac{\partial^{2} \delta \varphi}{\partial x^{2}}-\delta \varphi+3 \varphi_{s}^{2} \delta \varphi=0
$$

This equation can be solved using a separation of variables, by substituting the ansatz:

$$
\delta \varphi(t, x)=\sum_{i} \delta \psi_{i}(x) \cos \left(\omega_{i} t+\theta_{i}\right)
$$

The temporal phase differences $\theta_{i}$ between distinct excitation modes will be set to zero from here on without loss of generality. Inserting the ansatz into the linearized equation of motion results in a set of Schrödinger-like equations, $\hat{H} \delta \psi_{i}=E \delta \psi_{i}$, with:

$$
\begin{aligned}
\hat{H} & =-\frac{d^{2}}{d x^{2}}+3 \varphi_{s}^{2}-1, \\
E & =\omega_{i}^{2} .
\end{aligned}
$$

Thus, the original problem of solving the linearized equation of motion can be restated as the search for all eigenvalues $\omega_{i}^{2}$ and eigenstates $\delta \psi_{i}(x)$ of the linear operator $\hat{H}$. Notice however, that these eigenfunctions accurately describe the excitation modes only in the limit of small amplitude, where ignoring higher order terms in Eq. (4.20) is justified. The exponential dependence of $\delta \varphi$ on $\omega_{i}$ in Eq. (4.21) implies that any eigenstate of the Hamiltonian with eigenvalue $\omega_{i}^{2}<0$ corresponds 


\section{Introduction to solitary waves}

to an instability of $\varphi_{s}(x)$. If the initial configuration $\varphi_{s}$ is a stable, static solution to the equation of motion, such negative-energy excitation modes should not occur.

For Lagrangian densities with translational symmetry, there always exists a zeroenergy excitation that corresponds to a rigid translation of the initial state. To see this explicitly for the $\varphi^{4}$ theory, first notice that the Hamiltonian in Eq. (4.22) can be written in terms of the potential given by Eq. (4.13) as:

$$
\hat{H}=-\frac{\partial^{2}}{\partial x^{2}}+\frac{d^{2} U}{d \varphi^{2}} .
$$

Then, compare this to the spatial derivative of the static version of Eq. (4.2), which can be written as:

$$
-\frac{d^{2} \varphi^{\prime}}{d x^{2}}+\frac{d^{2} U}{d \varphi^{2}} \cdot \varphi^{\prime}=0 .
$$

Here, $\varphi^{\prime}=\partial \varphi / \partial x$ is the spatial derivative of the static field $\varphi(x)$. Any solution $\varphi_{s}$ to the equation of motion, is also a solution to Eq. (4.25). On the other hand, this equation may be interpreted as the Schrödinger equation $\hat{H} \varphi^{\prime}=E \varphi^{\prime}$ with zero energy and $\hat{H}$ defined by Eq. (4.24). We thus find that for every solution $\varphi_{s}$ to the equation of motion, there exists a zero-energy excitation $\delta \varphi(t, x) \propto \varphi_{s}^{\prime}$.

Focusing now on the specific case of kinks in the $\varphi^{4}$ theory, consider $\varphi_{s}$ to be the static kink configuration of Eq. (4.15), with $a=0$. The Hamiltonian whose eigenfunctions are the excitation modes of the kink, then becomes:

$$
\hat{H}=-\frac{d^{2}}{d x^{2}}-\frac{3}{\cosh ^{2}(x / \sqrt{2})}+2 .
$$

The eigenvalue equation for this specific Hamiltonian happens to be a well-known problem with an analytic solution [177]. There are two eigenfunctions, corresponding to two distinct excitation modes of the kink:

$$
\begin{array}{ll}
\delta \psi_{0}(x)=\left(\frac{3}{4 \sqrt{2}}\right)^{1 / 2} \frac{1}{\cosh ^{2}(x / \sqrt{2})} & \text { with } \omega_{0}=0, \\
\delta \psi_{1}(x)=\left(\frac{3}{2 \sqrt{2}}\right)^{1 / 2} \frac{\sinh ^{2}(x / \sqrt{2})}{\cosh ^{3}(x / \sqrt{2})} & \text { with } \omega_{1}=\sqrt{3 / 2} .
\end{array}
$$

The zero-energy solution $\delta \psi_{0}$ is proportional to $\varphi_{K}^{\prime}$, the spatial derivative of the kink solution. We thus recognise it as the translational mode that shifts the kink along the spatial axis. The mode at nonzero excitation energy, $\delta \psi_{1}$, corresponds to vibrations of the kink around its equilibrium shape, which do not affect the location at which the field value crosses zero. The vibrational mode at nonzero energy can 


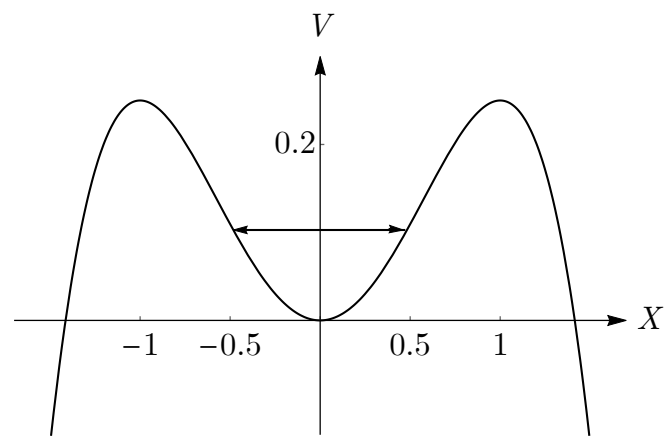

Figure 4.8: The plot of the Eq. (4.30) (up to a constant 0.25). The arrow shows the solution $\varphi(x)$ (after identification $X(T) \rightarrow \varphi(x)$ ) for $\varphi_{0}=0.5$ that corresponds to a dashed blue line in the Fig. 4.5 .

be excited during kink collisions, and enables the formation of resonance windows or quasi-long-lived wobbling kink [178, 179].

\section{B Elliptic sine solution}

To analytically find static solutions of the Eq. (4.14) in the $\varphi^{4}$ theory, we assume that there exists a stationary solution $\varphi(x)$ that must then obey the equation of motion with vanishing time derivatives:

$$
\frac{d^{2} \varphi}{d x^{2}}=\varphi^{3}-\varphi
$$

To get some feeling for this equation, notice that it is reminiscent of Newton's second law of motion, $-d V / d X=m d^{2} X / d T^{2}$, where the role of time $T$ is played by the coordinate $x$, and the position $X$ corresponds the field value $\varphi$. In other words, the configuration $\varphi(x)$ may be read as the trajectory $X(T)$ of a particle with mass $m=1$, subject to the potential:

$$
V(X)=-\frac{1}{4}\left(1-X^{2}\right)^{2}
$$

This potential is displayed in Fig. 4.8. For initial conditions $X(0)=X_{0}<X_{\max }$ and $d X / d T=0$, the particle motion will consist of periodic oscillations between $X_{0}$ and $-X_{0}$. These stable trajectories all correspond to static solutions of the $\varphi^{4}$ theory, after substituting back $X(T) \rightarrow \varphi(x)$. 


\section{Introduction to solitary waves}

We can find an analytic expression for the static field configurations by first integrating both sides of Eq. (4.29):

$$
\begin{aligned}
\frac{d^{2} \varphi}{d x^{2}}+\varphi-\varphi^{3} & =0 \\
\int d x \frac{d \varphi}{d x}\left(\frac{d^{2} \varphi}{d x^{2}}+\varphi-\varphi^{3}\right) & =\int d x \frac{d \varphi}{d x}(0), \\
\frac{1}{2}\left(\frac{d \varphi}{d x}\right)^{2}+\frac{1}{2} \varphi^{2}-\frac{1}{4} \varphi^{4} & =C .
\end{aligned}
$$

Here, $C$ is a constant of integration. Using intuition based on the particle trajectory analogy, we can choose boundary conditions such that $\varphi=\varphi_{0}$ at the point where $d \varphi / d x=0$. This yields $C=\varphi_{0}^{2} / 2-\varphi_{0}^{4} / 4$, and suggests separating the amplitude and spatial dependence of the field, by writing $\varphi(x)=\varphi_{0} \chi(x)$, with $|\chi(x)| \leq 1$. In terms of these new variables, Eq. (4.31) becomes:

$$
\begin{aligned}
\left(\frac{d \chi}{d x}\right)^{2} & =1-\chi^{2}-\frac{1}{2} \varphi_{0}^{2}\left(1-\chi^{4}\right) \\
& \equiv b^{2}\left(1-\chi^{2}\right)\left(1-k^{2} \chi^{2}\right) .
\end{aligned}
$$

In the final line, we introduced the definitions:

$$
k^{2}=\frac{\varphi_{0}^{2}}{2-\varphi_{0}^{2}}, \quad b^{2}=1-\frac{\varphi_{0}^{2}}{2} .
$$

Because we know from the particle trajectory analogy that there will be no stable solutions for $\left|\varphi_{0}\right|>1$, we always have $0 \leq k^{2} \leq 1$, and $1 / 2 \leq b^{2} \leq 1$. Integrating Eq. (4.34) now yields:

$$
\begin{aligned}
\left(\frac{d \chi}{d x}\right)^{2} & =b^{2}\left(1-\chi^{2}\right)\left(1-k^{2} \chi^{2}\right), \\
\int_{0}^{x^{\prime}} d x \frac{|d \chi / d x|}{\sqrt{\left(1-\chi^{2}\right)\left(1-k^{2} \chi^{2}\right)}} & =\int_{0}^{x^{\prime}} d x b \\
\int_{\chi(0)}^{\chi\left(x^{\prime}\right)} \frac{d \chi}{\sqrt{\left(1-\chi^{2}\right)\left(1-k^{2} \chi^{2}\right)}} & =b x^{\prime} .
\end{aligned}
$$

In the final line, we assumed that $d \chi / d x$ is positive over the integration interval. It is easily checked that the final solution we obtain below will be valid also along intervals where $d \chi / d x$ is negative, and that these intervals connect smoothly. Choosing $\chi(0)=0$ without loss of generality, and making the substitutions $\chi \equiv \sin (\psi)$ and 


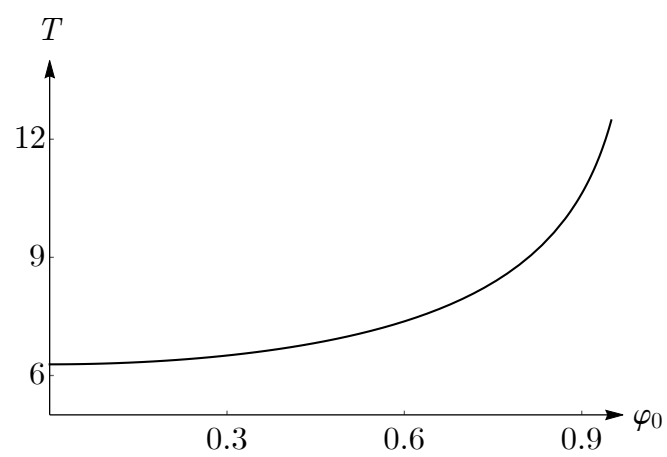

Figure 4.9: The dependence of the period $T$ given by Eq. (4.41) of the elliptic function on the parameter $\varphi_{0}$.

$C^{\prime} \equiv \arcsin \chi\left(x^{\prime}\right)$, Eq. (4.36) reduces to:

$$
\int_{0}^{C^{\prime}} \frac{d \psi}{\sqrt{\left(1-k^{2} \sin ^{2} \psi\right)}}=b x^{\prime} .
$$

The integral on the left hand side is a standard integral, known as the incomplete elliptic integral of the first, and is often denoted $\mathrm{F}\left(C^{\prime}, k\right)$ [180]. Using the known properties of the elliptic integral, Eq. (4.39) can be inverted, and yields the solution $\chi\left(x^{\prime}\right)=\operatorname{sn}\left(b x^{\prime}, k\right)$, with $\operatorname{sn}(b x, k)$ the elliptic sine [180]. The static solutions to the equation of motion for the $\varphi^{4}$ theory can thus finally be written as:

$$
\varphi_{s}(x)=\varphi_{0} \operatorname{sn}(b x, k) \text {. }
$$

For $\varphi_{0}<1$, these solutions are the elliptic sines shown in Fig. 4.5. For very small values of $\varphi_{0}$, the elliptic sine approaches $\sin (x)$, and the static solution corresponds to small sinusoidal oscillations around the unstable homogeneous solution $\varphi=0$, which it approaches in the limit $\varphi_{0} \rightarrow 0$.

To understand the opposite limit, of $\varphi_{0} \rightarrow 1$, notice that the period of the static solution $\varphi_{s}(x)$ is given by [180]:

$$
T=\frac{4 \mathrm{~F}(\pi / 2, k)}{\sqrt{1-0.5 \varphi_{0}^{2}}} .
$$

The period is plotted as a function of $\varphi_{0}$ in Fig. 4.9. As $\varphi_{0}$ approaches one, the period diverges. In terms of the particle trajectory analogue, this situation cor- 


\section{Introduction to solitary waves}

responds to the massive particle starting out precisely in an unstable equilibrium state on one of the potential maxima. If the particle at some point (spontaneously, or due to an infinitesimal perturbation) leaves the unstable maximum, it traverses the minimum in the potential and ends up at the opposite maximum at infinite time.

In terms of the field $\varphi_{s}(x)$, the same limiting behavior corresponds precisely to a kink configuration. This can be seen taking the limit $\varphi_{0} \rightarrow 0$ directly in Eq. (4.36), and using $k^{2} \rightarrow 1$ and $b \rightarrow 1 / \sqrt{2}$ :

$$
\begin{aligned}
\int_{0}^{\chi\left(x^{\prime}\right)} \frac{d \chi}{1-\chi^{2}} & =\frac{x^{\prime}}{\sqrt{2}}, \\
{\left[\frac{1}{2} \ln \left(\frac{1+\chi}{1-\chi}\right)\right]_{0}^{\chi\left(x^{\prime}\right)} } & =\frac{x^{\prime}}{\sqrt{2}}, \\
\chi\left(x^{\prime}\right) & =\tanh \left(x^{\prime} / \sqrt{2}\right) .
\end{aligned}
$$

Since we took $\varphi_{0} \rightarrow 1$, the final static solution $\varphi_{s}$ equals $\chi$, and we recover the equation for the kink, Eq. (4.15), centered at the origin. 


\section{Solitary wave propagation in me- dia with step-like inhomogeneities}

We study the evolution of solitary-wave solutions in $(1+1)$ dimensions within the sine-Gordon and the $\varphi^{4}$ field models propagating in media with step-like inhomogeneities. We choose two different protocols. First, we consider a step-like spatial dependence of the overall coupling constant in the potential, which physically corresponds to the junction of two homogeneous systems, e.g., a Josephson junction. Second, we explore a quench protocol that corresponds to a sudden change of the coupling constant in the whole of space. In both scenarios, we study the solitary wave and observe that its behavior can be predicted describing the latter as a relativistic particle. Most of predictions are model-independent meaning that the integrable structure of the sine-Gordon model is irrelevant and rather the general topological sector of the considered profiles is important.

\subsection{Introduction}

Solitary waves play an important role in several areas of physics, from particle physics and cosmology to (nonlinear) optics and condensed-matter theory, as we already discussed in Chapter 4.

The most elementary models that host solitary-wave solutions (solitons and kinks) are the $(1+1)$-dimensional sine-Gordon and $\varphi^{4}$ field theories. While the sine-Gordon model is integrable, the $\varphi^{4}$ model is not. Comparing the two models thus allows us to study a role of integrability in the step-like inhomogeneous setups. The solitons in the sine-Gordon equation can describe the magnetic flux quantum in a Josephson junction, thus, it can be realized in a real setup.

Here, we study the interaction of a solitary wave with a defect that is represented by an inhomogeneity of the coupling constant. More specifically, we focus on a 
spatial step-like dependence of the coupling constant, which equals to some constant value for one part of the space and another for the other one. Physically, such a situation corresponds to the junction of two systems with different parameters. We study conditions under which the wave is reflected, transmitted by the junction, or is locked in between two junctions. We also study soliton behavior in the homogeneous system with a quench. Then, the value of the coupling constant is suddenly changed in time. Our method is a numerical integration of the equation of motion for different initial conditions. To give theoretical predictions we use the fact that a solitary wave in the homogeneuous space has kinetic properties of a relativistic particle. The inhomogeneity can be attributed to the step-like spatial or temporal dependence of the particle's mass. We numerically observe that this is indeed the case: the solitary wave remains localized shape after interacting with the junction or after the quench. This robustness is rooted in the topological character of the field configurations and the smallness of the radiation modes. The similarity of the results for the sine-Gordon and the $\varphi^{4}$ models suggests that integrability is irrelevant for such type of phenomena.

The outline of this Chapter is the following: in Section 5.2, we introduce the steplike inhomogeneity in the scalar field. Then, in Sections 5.3.1-5.3.4 we present the results for quench in space for moving solitary wave and static wave on the border. In Section 5.3.5 we consider a locking of the moving solitary wave between two dense regions. Afterwards, in Section 5.4 we look at quench in time. Finally, in Section 5.5, we conclude.

\subsection{Step-like inhomogeneity in scalar fields}

One way to add a step-like inhomogeneity in the systems is to modify Eq. (4.1) and Eq. (4.2) in the following way:

$$
\begin{gathered}
\mathscr{L}=\frac{1}{2}\left(\frac{\partial \varphi}{\partial t}\right)^{2}-\frac{1}{2}\left(\frac{\partial \varphi}{\partial x}\right)^{2}-\Theta(t, x) U(\varphi), \\
\varphi_{t t}-\varphi_{x x}+\Theta(t, x) \frac{d U}{d \varphi}=0
\end{gathered}
$$

where

$$
\Theta(t, x)=\zeta_{1} \theta(-x)+\zeta_{2} \theta(x), \quad-\infty<t<\infty, \quad \text { case }(\mathrm{i})
$$

or

$$
\Theta(t, x)=\theta(-t)+\zeta \theta(t), \quad-\infty<x<\infty, \quad \text { case (ii), }
$$

with $\theta(x)$ and $\theta(t)$ denoting the Heaviside step function. In the case (i), we obtain two homogeneous parts of space, with different prefactors $\zeta_{1}$ and $\zeta_{2}$, forming a junc- 
tion at the point $x=0$. The case (ii), is nothing but a quench (the sudden change) of the coupling constant at the moment $t=0$ by the factor $\zeta$, homogeneously in the whole space. Further, we take into account only $\zeta_{1} \geq 0, \zeta_{2} \geq 0$, and $\zeta \geq 0$ to remain in the class of nonnegative potentials $U(\varphi)$.

The equation of motion Eq. (5.2) reads for the sine-Gordon model as

$$
\varphi_{t t}-\varphi_{x x}+\Theta(t, x) \sin \varphi=0
$$

and for the $\varphi^{4}$ model as

$$
\varphi_{t t}-\varphi_{x x}+\Theta(t, x)\left(\varphi^{3}-\varphi\right)=0
$$

For the coupling constant scaled by the factor $\zeta$ the soliton $\varphi_{S}$ and the kink $\varphi_{K}$ read as

$$
\varphi(t, x)=4 \arctan \left[\exp \left(\sqrt{\zeta} \frac{x+a-v t}{\sqrt{1-v^{2}}}\right)\right]
$$

and

$$
\varphi(t, x)=\tanh \left(\sqrt{\zeta} \frac{x+a-v t}{\sqrt{2\left(1-v^{2}\right)}}\right),
$$

correspondingly. The trivial static solutions, namely vacua and unstable maxima of $U(\varphi)$ are not influenced by the change of a coupling constant in both theories.

We want to study the evolution of a solitary wave for the sine-Gordon and the $\varphi^{4}$ models. In all scenarios, we solve Eq. (5.2) numerically with a solitary wave, as initial condition, that is solution of corresponding equation of motion with $\zeta_{1}=$ $\zeta_{2}=\zeta=1$ (see Appendix 5.A). Hereinafter, just mentioned initial conditions we represent schematically as

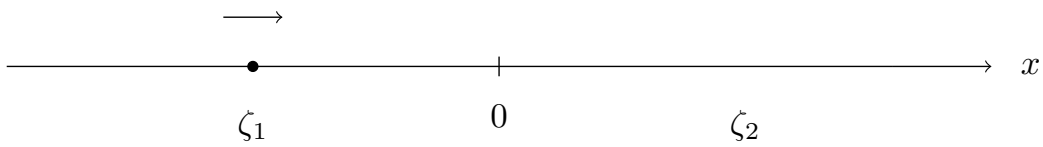

The center of the solitary wave is depicted with a black dot, and the arrow shows the direction of movement.

We characterize the wave evolution by its final velocity $v_{f}$ (its sign and value). If the wave is reflected, then $v_{f}<0$, while the wave going through a border yields $v_{f}>$ 0 . The solitary wave under consideration is localized in both systems, consequently, one may just focus on its center $x_{c}$ as a function of $t$ and check the final velocity $v_{f}$ of the wave

$$
v_{f}=\frac{x_{c}\left(t_{2}\right)-x_{c}\left(t_{1}\right)}{t_{2}-t_{1}}
$$

where $t_{1}$ and $t_{2}$ are some separated moments in time after interaction with a junction 
in the system (see also Appendix 5.B).

\subsection{Quench in space}

\subsubsection{Moving solitary waves: $\zeta_{1}=1$ and $0<\zeta_{2}<\infty$}

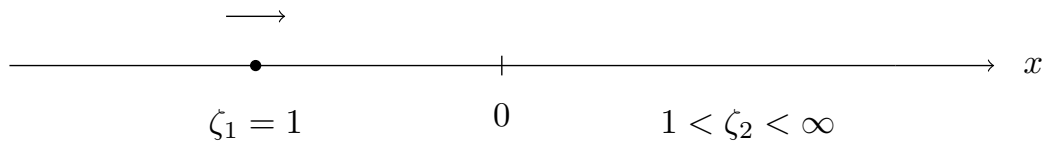

We start with junction of two homogeneous systems with step-like nonuniformity by taking Eq. (5.3) with $\zeta_{1}=1$ and $1<\zeta_{2}<\infty$ in Eq. (5.2). We want to understand the behavior of the moving soliton $\varphi_{S}$ or the moving kink $\varphi_{K}$, that we put at $x=-7$ at $t=0$ and send with some initial velocity $0<v_{i n}<1$ from left to right. We observe that the dynamics for the sine-Gordon and the $\varphi^{4}$ theories are similar.

For small initial velocities the kinetic energy is not enough to overcome the potential barrier, so we observe an elastic reflection resulting with a final velocity $v_{f}=-v_{i n}$. The turning point of the soliton/kink lies close to the junction, and depending on the initial velocity $v_{i n}$, can be either in the $\zeta_{1}$ area (see Fig. 5.1) or in $\zeta_{2}$ area (see Fig. 5.2), this way exhibiting a finite penetration length in the more dense media. We plot the dependence of the minimal distance measured from the junction, as the function of $\zeta_{2}$, for the fixed initial velocities in Fig. 5.3 for both systems. By fitting the data we conclude that there is an exponential decay with the parameter $\zeta_{2}$. In Fig. 5.4 we show the evolution of waves for the sine-Gordon and the $\varphi^{4}$ models for small initial velocities.

For high initial velocities, the solitary wave is completely transmitted and continues its propagation indefinitely. After entering the area with $\zeta_{2}$ the initial solitary wave given by Eq. (4.11) or Eq. (4.16), it is no longer an exact solution of Eq. (5.5) or Eq. (5.6), correspondingly. Thus, for the systems it is energetically favorable to modify the solitary wave from Eq. (4.11) or Eq. (4.16) to Eq. (5.7) or Eq. (5.8) that are the exact solutions of Eq. (5.5) or Eq. (5.6) at $x \geq 0$. This process is accompanied by a velocity drop and occurrence of small waves (radiation) on the surface of the soliton or the kink. In Fig. 5.5 we show the evolution of waves for the sine-Gordon and the $\varphi^{4}$ models, and in Fig. 5.6 we show the dependence of final velocity on the initial one.

The presence of two different processes depending on the inial velocities means that there is a critical velocity $v_{c r}$ that separates them. The particular values of $v_{c r}$ depend on the value $\zeta_{2}$, however, we notice that they are independent of the choice 


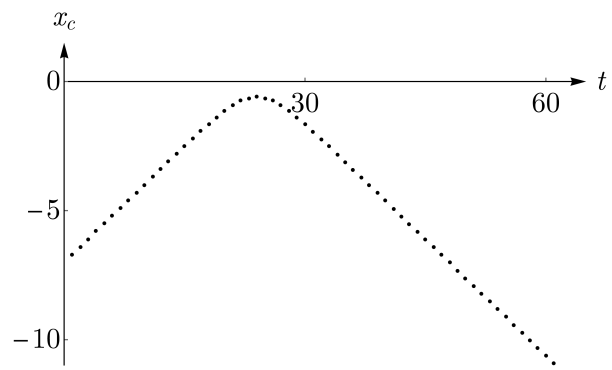

(a)

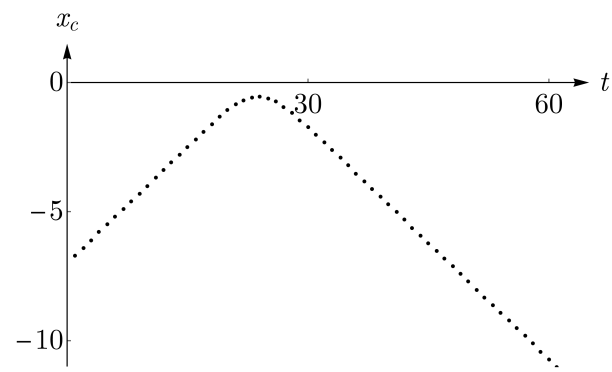

(b)

Figure 5.1: The dependence of the center of the solitary wave $x_{c}$ on time for fixed $\zeta_{1}=1, \zeta_{2}=1.5$, and $v_{i n}=0.3$. (a) For the sine-Gordon model. (b) For the $\varphi^{4}$ model. The maximums of the functions are $x_{c}=-0.60$ for (a) and $x_{c}=-0.55$ for (b).

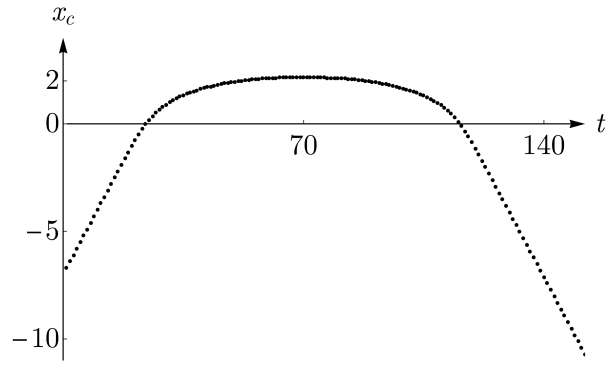

(a)

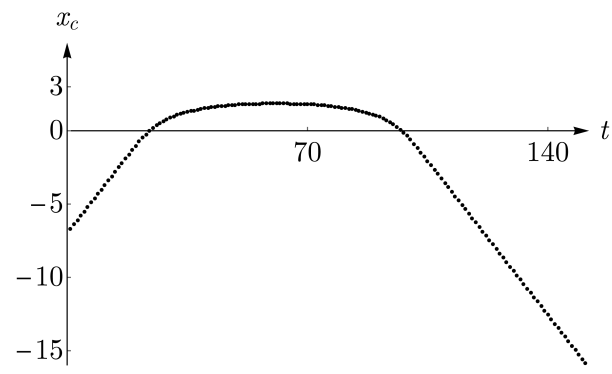

(b)

Figure 5.2: The dependence of the center of the solitary wave $x_{c}$ on time for fixed $\zeta_{1}=1, \zeta_{2}=1.1$, and $v_{i n}=0.3$. (a) For the sine-Gordon model. (b) For the $\varphi^{4}$ model.

of the model (see Fig. 5.7). This result may be explained by energy conservation. For presence of a piecewise constant given by Eq. (5.3) in the system we rewrite Eq. (4.3)

$$
E[\varphi]=\int_{-\infty}^{+\infty}\left[\frac{1}{2}\left(\frac{\partial \varphi}{\partial t}\right)^{2}+\frac{1}{2}\left(\frac{\partial \varphi}{\partial x}\right)^{2}+\left[\zeta_{1} \theta(-x)+\zeta_{2} \theta(x)\right] U(\varphi)\right] d x
$$

that is conserved in time (see Appendix 5.A). The general form of a solitary wave 
5 Solitary wave propagation in media with step-like inhomogeneities

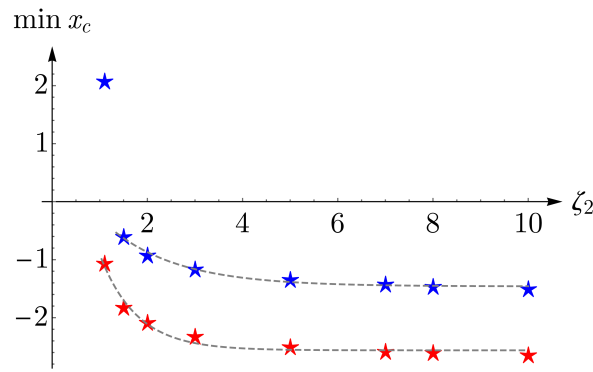

(a)

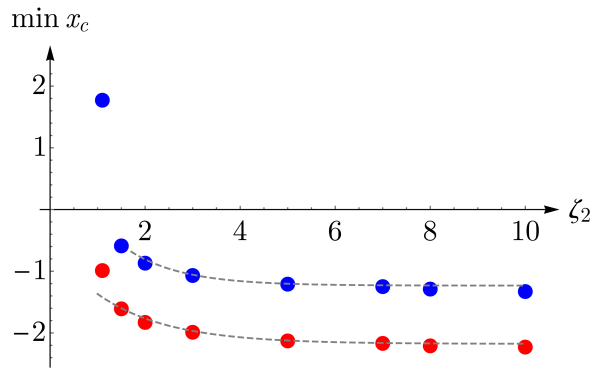

(b)

Figure 5.3: The dependence of the minimal distance, on which the waves are coming to the region with $\zeta_{2}$ for fixed values of subcritical initial velocities $v_{\text {in }}=0.1$ (red stars and dots) and $v_{i n}=0.3$ (blue stars and dots). Here, $\zeta_{1}=1$. (a) For the sine-Gordon model. (b) For the $\varphi^{4}$ model. The gray line on both panels represents the fitting with $a+b \exp \left(c \cdot \zeta_{2}\right)$, where $a, b$, and $c$ are some parameters of fitting that are different for each of these lines.

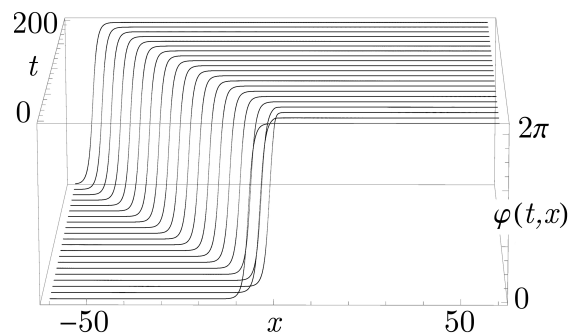

(a)

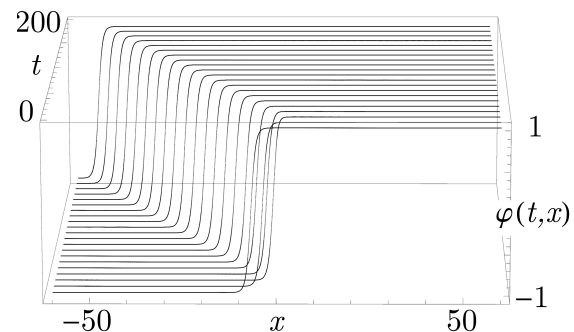

(b)

Figure 5.4: Solitary wave with subcritical velocity $v_{i n}<v_{c r}$ incident from the left onto the barrier and its elastic back-reflection for: (a) the sineGordon model with $\zeta_{1}=1, \zeta_{2}=5$, and $v_{i n}=0.3$; (b) the $\varphi^{4}$ model with $\zeta_{1}=1, \zeta_{2}=2$, and $v_{i n}=0.3$.

(Eq. (4.11) or Eq. (5.7) or Eq. (4.16)) or Eq. (5.8)) is

$$
\varphi_{1,2}(x, t)=F\left(\sqrt{\zeta_{1,2}} \frac{x-v_{1,2} t}{\sqrt{1-v_{1,2}^{2}}}\right),
$$

where $\varphi_{1}(x, t)$ and $\varphi_{2}(x, t)$ are the solutions of Eq. (5.2) for two parts of space with 


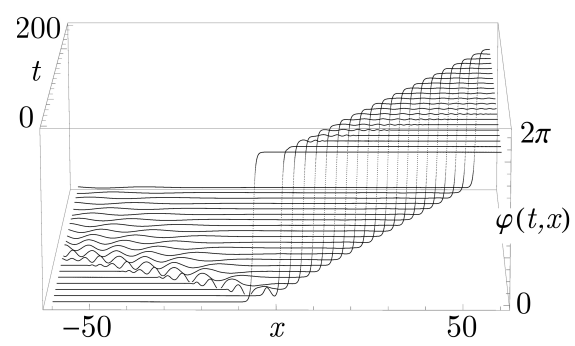

(a)

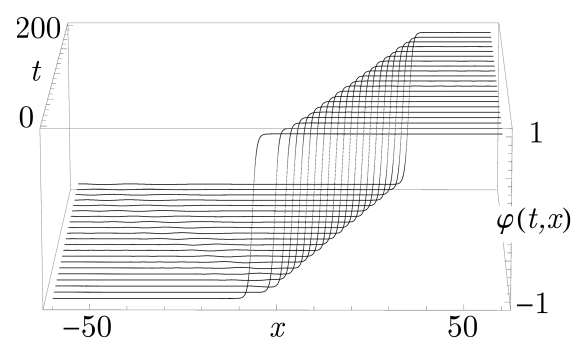

(b)

Figure 5.5: Solitary wave with supercritical velocity $v_{i n}>v_{c r}$ incident from the left onto the barrier and its transmission: (a) the sine-Gordon model with $\zeta_{1}=1, \zeta_{2}=5$, and $v_{i n}=0.91$; (b) the $\varphi^{4}$ model with $\zeta_{1}=1$, $\zeta_{2}=2$, and $v_{\text {in }}=0.80$.

prefactors $\zeta_{1}$ and $\zeta_{2}$ in the equation of motion respectively. Here, $v_{1}=v_{i n}$ and $v_{2}=v_{f}$. Now let us consider energy for $x<0$ at $t=0$. Specifically, for Eq. (5.11), the energy given by Eq. (5.10) can be written as

$$
E_{1}=\frac{\sqrt{\zeta_{1}}}{\sqrt{1-v_{1}^{2}}} M
$$

where $M$ reads as

$$
M=\int_{-\infty}^{\infty} d x\left[\frac{1}{2}\left(\frac{d F}{d x}\right)^{2}+U(F(x))\right] .
$$

The only assumption made here is that we can extend the integration domain form $(-\infty, 0]$ to $(-\infty, \infty)$, because the profile is highly localized. This is true for all times except the moment of the collision. Similarly for the right part of the space

$$
E_{2}=\frac{\sqrt{\zeta_{2}}}{\sqrt{1-v_{2}^{2}}} M
$$

Neglecting the radiation that appeared during the crossing process we can just equate $E_{1}=E_{2}$ due to energy conservation and obtain $v_{f}$, namely

$$
v_{f}^{\text {theor }}=\sqrt{\frac{\zeta_{2}}{\zeta_{1}}\left(v_{i n}^{2}-\frac{\zeta_{2}-\zeta_{1}}{\zeta_{2}}\right)}=\sqrt{\frac{\zeta_{2}}{\zeta_{1}}\left(v_{i n}^{2}-1\right)+1} .
$$

We see that this equation always has solutions for $\zeta_{1}>\zeta_{2}$, but in the opposite case 
5 Solitary wave propagation in media with step-like inhomogeneities

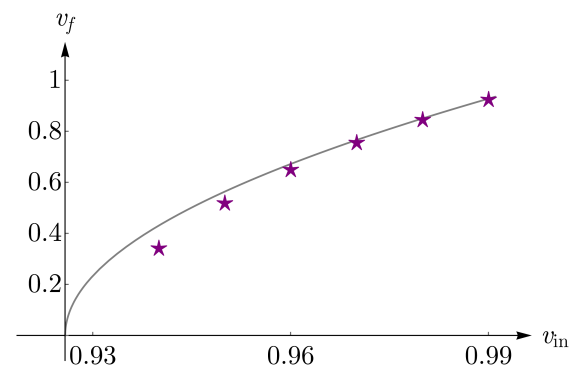

(a)

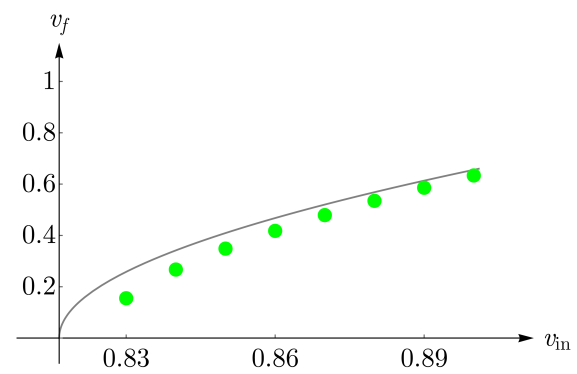

(b)

Figure 5.6: The dependencies of the final velocities $v_{f}$ given by Eq. (5.9) on supercritical initial velocity $v_{i n}>v_{c r}$ for $\zeta_{1}=1$ and fixed $\zeta_{2}$. (a) For the sine-Gordon model, $\zeta_{2}=7$. (b) For the $\varphi^{4}$ model, $\zeta_{2}=3$. The gray line on both panels represents the theoretical prediction $v_{f}^{\text {theor }}$ given by Eq. (5.15).

$v_{\text {in }}$ should be bigger than some critical value

$$
v_{c r}^{\text {theor }}=\sqrt{\frac{\zeta_{2}-\zeta_{1}}{\zeta_{2}}}=\sqrt{1-\frac{\zeta_{1}}{\zeta_{2}}} .
$$

This formula was already obtained in [39] (Eq. 5.23), however, it has a typo that we correct here. We overlap $v_{c r}$ in Fig. 5.7 that showed itself independent of the model with theoretically predicted value $v_{c r}^{\text {theor }}$ and see a perfect match.

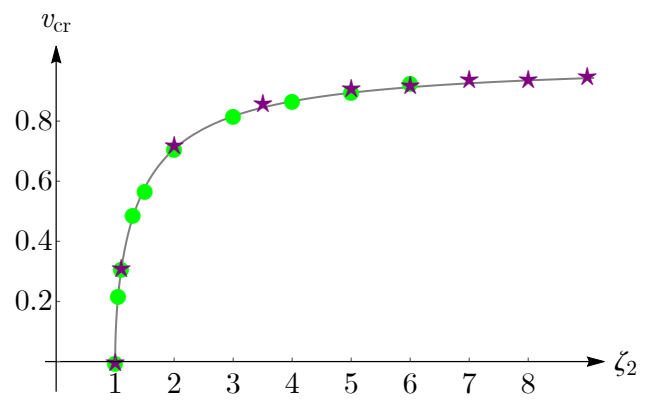

Figure 5.7: The dependence of the critical velocity $v_{c r}$ for the inhomogeneity jump from $\zeta_{1}=1$ to $\zeta_{2}>1$ in the sine-Gordon model (purple stars), the $\varphi^{4}$ model (green dots), and the theoretical prediction $v_{c r}^{\text {theor }}$ given by Eq. (5.16) (gray line). 
Then, we compare the theoretical prediction for the final velocity given by Eq. (5.15) to the numerically obtained values for some chosen values of $\zeta_{2}$ for the sine-Gordon and the $\varphi^{4}$ theories. The results are presented in Fig. 5.6. Contrary to the perfect match for the critical value of the velocity, the value for $v_{f}$ somewhat deviates for a theoretical prediction (see Fig. 5.6). The most significant deviation for the velocities happens just above the critical velocity. In this case our main assumption of the solitary wave being in either half of the space is violated. In particular, from the theoretical prediction one would expect for $v_{i n}=v_{c r}^{\text {theor }}$, implying the solitary wave would stick to the boundary. However, we see that it never happens, and in fact for $v_{i n}=v_{c r}^{\text {theor }}$ is still reflected. The existence of the solution with $v_{f}=0$ would represent a static solution localized on the boundary. In Section 5.3 we try to construct this solution artificially, and observe that it always acquires some finite velocity.

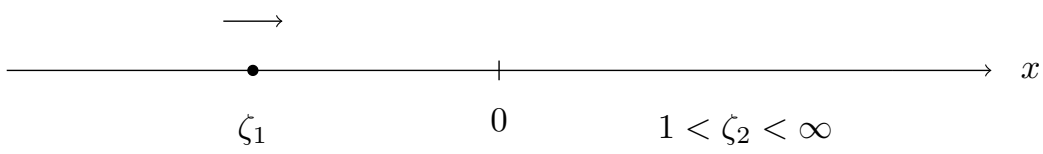

One can notice, that the critical velocity $v_{c r}^{\text {theor }}$ defined in the Eq. (5.16) depends not on $\zeta_{1}$ and $\zeta_{2}$ separately, but on its ratio $\zeta_{1} / \zeta_{2}$. In order to check it numerically, we choose different values of $\zeta_{1}$ and $\zeta_{2}$, while the keeping ratio $\zeta_{1} / \zeta_{2}$ constant for both models. Changing the initial conditions appropriately (from Eq. (4.11) to Eq. (5.7) and Eq. (4.16) to Eq. (5.8) for corresponding value of $\zeta_{1}$ ) we verify for the sine-Gordon model that the critical velocity for $\zeta_{1}=3$ and $\zeta_{2}=30$ is the same as for $\zeta_{1}=1$ and $\zeta_{2}=10$, and that $\zeta_{1}=2$ and $\zeta_{2}=6$ is equivalent to $\zeta_{1}=1$ and $\zeta_{2}=3$ for the $\varphi^{4}$ model.

\subsubsection{Moving solitary waves: $\zeta_{1}=1$ and $0<\zeta_{2}<1$}

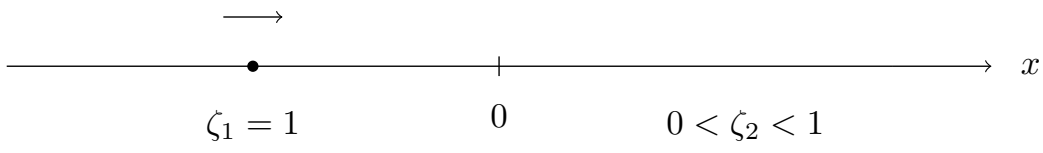

The case $\zeta_{2}<\zeta_{1}$ can be treated similarly, since Eq. (5.15) and Eq. (5.16) we derived without any restrictions on $\zeta_{1}$ and $\zeta_{2}$. In this case, we see numerically that the solitary wave is always transmitted regardless of the values of the initial velocity with $v_{f}>v_{i n}$. This can be shown using Eq. (5.15), namely

$$
\left(v_{f}^{\text {theor }}\right)^{2}=\frac{\zeta_{2}}{\zeta_{1}}\left(v_{i n}^{2}-1\right)+1>v_{i n}^{2} \text { or }\left(1-\frac{\zeta_{2}}{\zeta_{1}}\right)\left(1-v_{i n}^{2}\right)>0 \text { or } \zeta_{2}<\zeta_{1} \text {. }
$$


5 Solitary wave propagation in media with step-like inhomogeneities

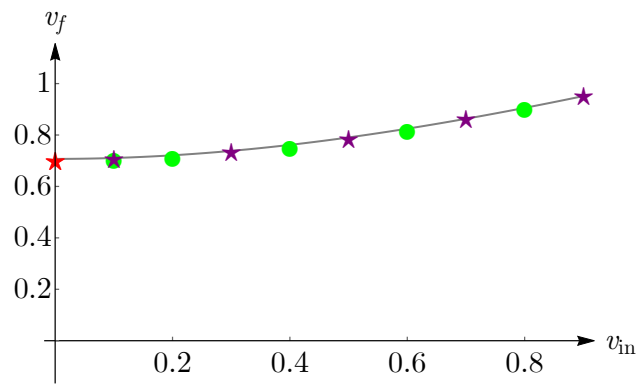

Figure 5.8: The dependence of the final velocity $v_{f}$ given by Eq. (5.9) for the inhomogeneity jump from $\zeta_{1}=1$ to $\zeta_{2}=0.5$ in the sine-Gordon model (purple stars for $a=7$, red star for $a=2$ ), the $\varphi^{4}$ model (green dots), and the theoretical prediction $v_{f}^{\text {theor }}$ given by Eq. (5.15) (gray line).

In Fig. 5.8 we plot the numerically obtained dependencies of the final velocities $v_{f}$ on initial velocities $v_{i n}$ for $\zeta_{2}<\zeta_{1}$ along with the theoretically predicted curve given by Eq. (5.15) for both systems. We see the perfect match between theoretical predictions and numerical results. Also, we notice that

$$
v_{f}^{\text {theor }}\left(v_{i n}=0\right)=\sqrt{1-\frac{\zeta_{2}}{\zeta_{1}}},
$$

which means that the less dense area sucks the static wave inside. To check it, we take the soliton with $a=2$ (to fix the time of the evolution) for $\zeta_{2}=0.5$, for which we observe this effect. In Fig. 5.8 we add the value of the gained velocity in this case.

\subsubsection{Moving solitary waves: $\zeta_{1}=1$ and $\zeta_{2}=0$}

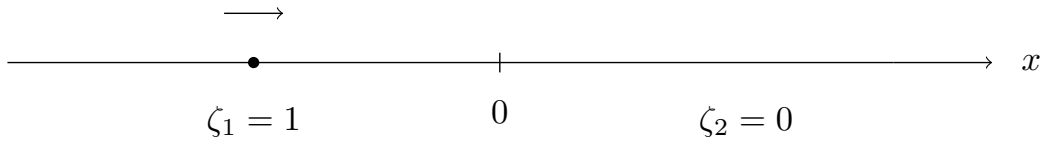

One can also apply Eq. (5.15) in the case $\zeta_{2}=0$. This value of $\zeta_{2}$ corresponds to the tunneling of the solitary wave to the vacuum. According to Eq. (5.15) the wave should be accelerated to the highest possible final velocity $v_{f}^{\text {theor }}=1$ regardless of the value of initial velocity. In numerical simulations we observe a perfect match with this prediction. The profile in the vacuum keeps its soliton-/kink-like form, 
which can be seen by taking the simultaneous limit $v \rightarrow 1$ and $\zeta_{2} \rightarrow 0$ in Eq. (5.7) or Eq. (5.8). This form is allowed by the conservation of the topological charge

$$
Q=\int_{-\infty}^{+\infty} \partial_{x} \varphi d x=\varphi(+\infty)-\varphi(-\infty)
$$

It gets its quantized value $2 \pi$ for the sine-Gordon model and two for the $\varphi^{4}$ model in the "dense" half of space, and keeps its value even in the vacuum due to conservation of this quantity

$$
\partial_{t} Q=\int_{-\infty}^{+\infty} \partial_{x} \partial_{t} \varphi d x
$$

where $\partial_{t} \varphi(-\infty)=\partial_{t} \varphi(+\infty)$ by constraint, thus, $\partial_{t} Q=0$. The waves start their motion from the area with $\zeta_{1} \neq 0$, where the topological charge is fixed by the potential $U(\varphi)$, and its conservation in time yields to keeping the original structure even after the transmission to the area with $\zeta_{2}=0$.

\subsubsection{Static solitary waves on border}

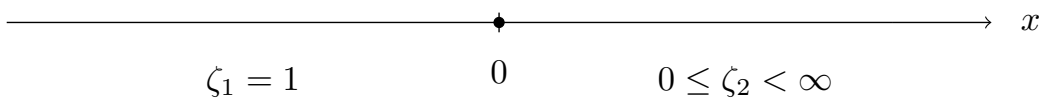

We continue considering a junction of two homogeneous systems with step-like nonuniformity by taking Eq. (5.2) and Eq. (5.3) with $\zeta_{1}=1$ and $0 \leq \zeta_{2}<\infty$. We take as the initial profile the originally static soliton or kink that is located exactly at the junction. More specifically, we take $\varphi_{S}$ or $\varphi_{K}$ given by Eq. (4.11) or Eq. (4.16) respectively, and put it at $x=0$ at $t=0$ with $v_{\text {in }}=0$.

The subsequent evolution looks similar for the soliton and the kink even on a quantitative level. The wave moves always into the less dense area that is a region with lower potential energy. For $\zeta_{2}<\zeta_{1}$ we observe the transmission to the right side of space with $\zeta_{2}$ (see Fig. 5.9), while for $\zeta_{2}>\zeta_{1}$ the wave is reflected into the area $\zeta_{1}$ (see Fig. 5.10). Thus, the solitary wave always gains some kinetic energy and keeps its localized form because of the topological constraint, in contrast to [181] when for similar systems a wave could stick near the step-like inhomogeneity. Only for $\zeta_{1}=\zeta_{2}$ the originally static solitary wave remains motionless, because the whole space is homogeneous, and Eq. (4.11) or Eq. (4.16) are the exact solutions of the corresponding equation of motion. In Fig. 5.11 we present numerically obtained dependencies of final velocities $v_{f}$ on value of $\zeta_{2}$ for the sine-Gordon and the $\varphi^{4}$ models. 
5 Solitary wave propagation in media with step-like inhomogeneities

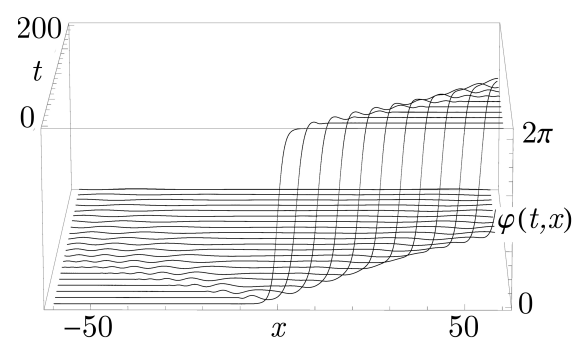

(a)

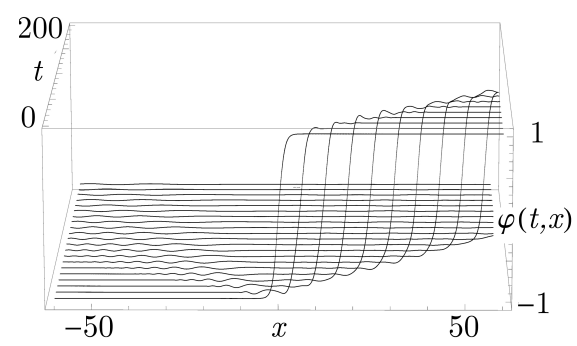

(b)

Figure 5.9: Static solitary wave on the border between $\zeta_{1}=1$ and $\zeta_{2}<1$ : (a) the sine-Gordon model with $\zeta_{2}=0.5$; (b) the $\varphi^{4}$ model with $\zeta_{2}=0.4$.

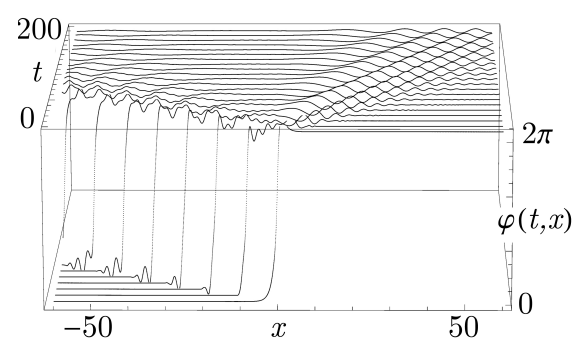

(a)

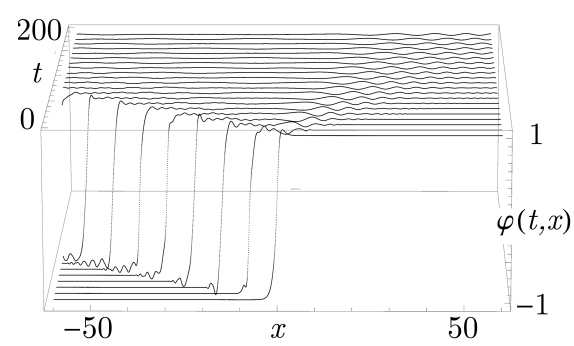

(b)

Figure 5.10: Static solitary wave on the border between $\zeta_{1}=1$ and $\zeta_{2}>1$ : (a) the sine-Gordon model with $\zeta_{2}=7$; (b) the $\varphi^{4}$ model with $\zeta_{2}=4$.

We can verify this result analytically using Eq. (5.10). We take profile in the form given by Eq. (5.11) with $\zeta_{1}$ for $-\infty<x<0$ and with $\zeta_{2}$ for $0 \leq x<+\infty$. Numerically this is implemented by taking the first and the second layer in the initial conditions (see Appendix 5.A), which yields $\partial_{t} \varphi=0$ in the initial moment. After substitution Eq. (5.11) in Eq. (5.10) with $\partial_{t} \varphi=0$ and integrating with $\sqrt{\zeta} x \rightarrow x$, one obtains

$$
\sqrt{\zeta_{1}} \int_{-\infty}^{0} d x[\ldots]+\sqrt{\zeta_{2}} \int_{0}^{+\infty} d x[\ldots]=\frac{M}{2}\left(\sqrt{\zeta_{1}}+\sqrt{\zeta_{2}}\right)
$$

where dots represent the integrand in Eq. (5.13), and $M$ is given by Eq. (5.13). This is an expression for energy at $t=0$. The total energy is conserved, and we assume that its value comes mainly from the localized wave that moves in the less dense area. So, we consider two separate cases, $\zeta_{1}>\zeta_{2}$ and $\zeta_{1}<\zeta_{2}$, for which the 
conservation law reads

$$
\frac{M}{2}\left(\sqrt{\zeta_{1}}+\sqrt{\zeta_{2}}\right)=\frac{M \sqrt{\min \left[\zeta_{1}, \zeta_{2}\right]}}{\sqrt{1-v_{f}^{2}}} .
$$

Then, the final velocity can be presented as

$$
\left(v_{f}^{\text {theor }}\right)^{2}=1-\frac{4 \min \left[\zeta_{1}, \zeta_{2}\right]}{\left(\sqrt{\zeta_{1}}+\sqrt{\zeta_{2}}\right)^{2}} .
$$

The sign for $v_{f}$ is such that the wave moves in the less dense area. We take $\zeta_{1}$ for $\zeta_{1}<\zeta_{2}$, then $v_{f}<0$ and $\zeta_{2}$ for $\zeta_{1}>\zeta_{2}$, then $v_{f}>0$, finally,

$$
v_{f}^{\text {theor }}=\operatorname{sgn}\left[\zeta_{1}-\zeta_{2}\right] \sqrt{1-\frac{4 \min \left[\zeta_{1}, \zeta_{2}\right]}{\left(\sqrt{\zeta_{1}}+\sqrt{\zeta_{2}}\right)^{2}}} .
$$

This result proofs that the dynamics of the solitary wave does not depend on the particular model. We compare the numerically obtained $v_{f}$ with the theoretical prediction $v_{f}^{\text {theor }}$ in Fig. 5.11 and see perfect agreement. Note that existence of a force pushing the fluxons in Josephson junction in the direction of smaller self-energy in the presence of a temperature gradient was earlier seen in experiments [182].

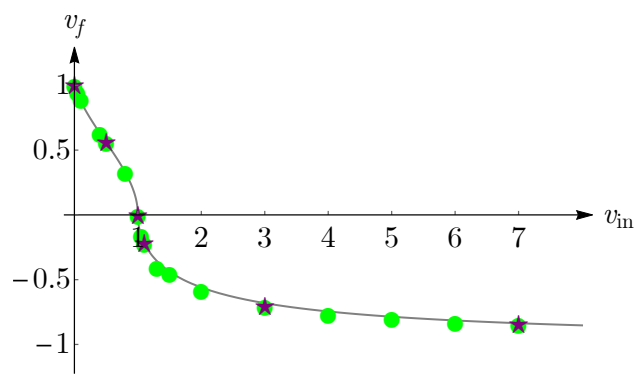

Figure 5.11: The dependence of the final velocity $v_{f}$ given by Eq. (5.9) for the originally static solitary wave on the border for the nonuniformity jump from $\zeta_{1}=1$ to $0 \leq \zeta_{2}<\infty$ in the sine-Gordon model (purple stars), the $\varphi^{4}$ model (green dots), and the theoretical prediction given by Eq. (5.24) (gray line). 


\subsubsection{Mirror reflection}

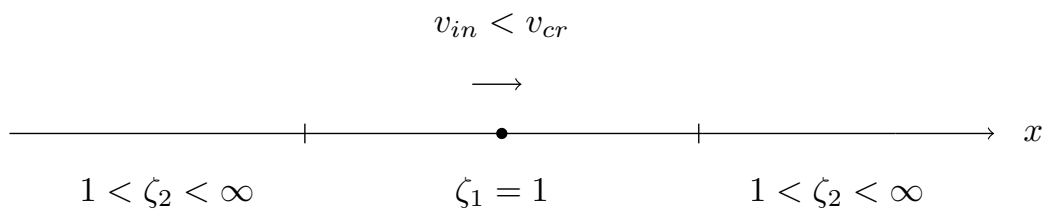

Now, we consider the junction of three homogeneous systems with step-like spatial nonuniformity by modifying Eq. (5.3) in a way

$$
\Theta(t, x)=\zeta_{2} \theta(|x|-b)+\zeta_{1} \theta(b-|x|)
$$

for $-\infty<t<\infty$. Here, $b>0$ defines the boundaries of the sections and is assumed to be much larger than the width of solitary waves $b \gg l_{S}$ or $b \gg l_{K}$. We set initial profile in the middle section in a form of the soliton or the kink moving with the subcritical velocity $0<v_{i n}<v_{c r}\left(v_{c r}\right.$ is defined by Eq. (5.16)). For such a choice of $v_{i n}$ the solitary wave would reflect from the boundaries at $x= \pm b$, so it cannot escape the middle section and moves freely inside as a photon between two mirrors. We check this behavior for both the sine-Gordon and the $\varphi^{4}$ models (see Fig. 5.12). Despite the small-amplitude ripples on the wave profile, after seven cycles the profile keeps its initial form and velocity, within the margin of error. Therefore, we obtain a long-lived solution that is oscillating between two regions in the space with a constant speed.

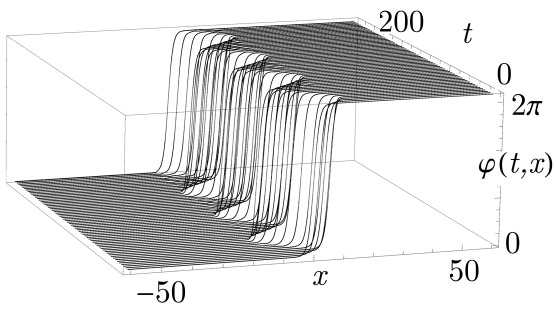

(a)

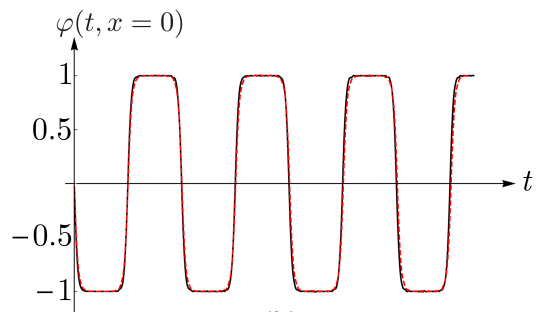

(b)

Figure 5.12: The moving solitary wave is locked in the region with $\zeta_{1}=1$ between two regions with $\zeta_{2}>1$ : (a) the sine-Gordon model with $\zeta_{2}=5$; (b) the comparison of dynamics in the sine-Gordon (red dashed line, a scaled data) and the $\varphi^{4}$ (black solid line) models with $\zeta_{2}=5$. 


\subsection{Quench dynamics}

In this section, we consider a nonequilibrium situation when the prefactor in front of the potential $U(\varphi)$ is rapidly changed (see Eq. (5.4)). This protocol is similar to the other quench protocols that effectively correspond to the re-scaling of $t$ and $x$ [183-186], since the coupling constant in the potential can be removed by such re-scaling. The main difference is, however, that due to the topological constraint there is no multiple soliton creation after the quench contrary to the nonlinear Schrödinger equation [183, 184], where radiation can accumulate the finite phase difference over the system [185].

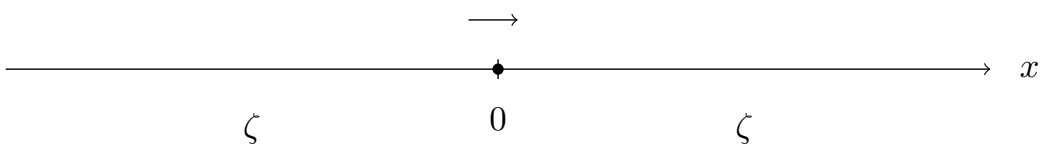

We consider the quench parameter $0<\zeta<\infty$ for both the sine-Gordon and the $\varphi^{4}$ models. As initial condition we consider the solitary wave given by Eq. (4.11) or Eq. (4.16) located at $x=0$ at $t=0$ and moving from left to right with some initial velocity $v_{i n}$.

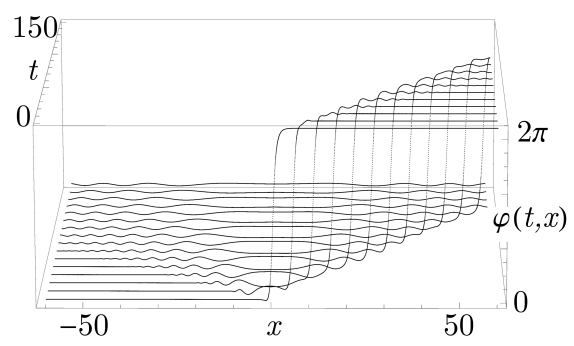

(a)

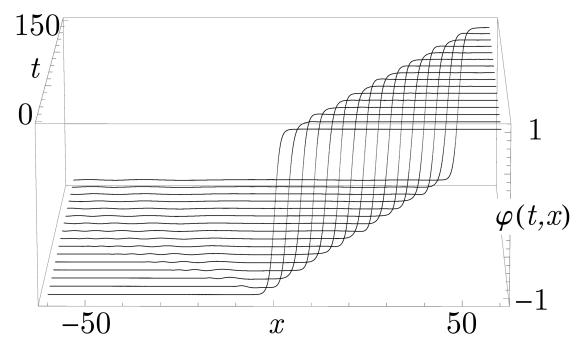

(b)

Figure 5.13: The solitary wave evolution for quench in time: (a) the sine-Gordon model with $\zeta=2.50$ and $v_{\text {in }}=0.7$; (b) the $\varphi^{4}$ model with $\zeta=0.75$ and $v_{i n}=0.3$.

In this setup, the profile given by Eq. (4.11) or Eq. (4.16) is no longer an exact solution of the corresponding equation of motion, thus, the evolution is accompanied by the emission of small waves. This small amplitude waves can be interpreted as plasma waves on top of fluxon in the Josephson junction (see Section 4.4 in Chapter 4). We see that the wave always conserves its original direction of motion, because the whole space is homogeneous, which means that there is no anymore less or more preferred area for wave motion. In Fig. 5.13 we present the profile of solitary waves 
5 Solitary wave propagation in media with step-like inhomogeneities

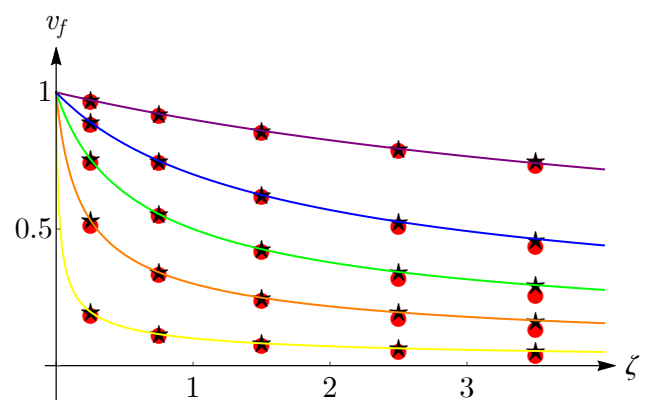

Figure 5.14: The dependence of the final velocity $v_{f}$ given by Eq. (5.9) on $\zeta$ for the quench in the sine-Gordon model (stars), the $\varphi^{4}$ model (dots), and the theoretical prediction given by Eq. (5.29) (lines). Parameter values: yellow for $v=0.1$, orange for $v=0.3$, green for $v=0.5$, blue for $v=0.7$, and purple for $v=0.9$.

for one chosen value of $\zeta$ and $v_{i n}$ for both models. The numerical measurement of the final velocities $v_{f}$ yields Fig. 5.14.

Like in Section 5.3 we notice the independence of the results on the choice of a particular model. To obtain an analytical prediction for $v_{f}$ for this we use that the momentum

$$
P=\int_{-\infty}^{+\infty} \partial_{t} \varphi \partial_{x} \varphi d x=\text { const }
$$

is conserved for this constraint, and substitution Eq. (5.11) yields

$$
\frac{-\zeta_{1,2} v_{1,2}}{1-v_{1,2}^{2}} \int_{-\infty}^{+\infty}\left[F^{\prime}\left(\sqrt{\zeta_{1,2}} \frac{x-v_{1,2} t}{\sqrt{1-v_{1,2}^{2}}}\right)\right]^{2} d x=\frac{-\sqrt{\zeta_{1,2}} v_{1,2}}{\sqrt{1-v_{1,2}^{2}}} \int_{-\infty}^{+\infty} F^{\prime}(y) d y
$$

In the considered case, we have $\zeta_{1}=1$ (according to the initial condition given by Eq. (4.11) or Eq. (4.16)), $\zeta_{2}=\zeta, v_{1}=v_{i n}$, and $v_{2}=v_{f}$. Then, the law of momentum conservation reads

$$
\frac{v_{i n}}{\sqrt{1-v_{i n}^{2}}}=\frac{\sqrt{\zeta} v_{f}}{\sqrt{1-v_{f}^{2}}}
$$

or

$$
v_{f}^{\text {theor }}=\frac{v_{i n}}{\sqrt{v_{i n}^{2}+\zeta\left(1-v_{i n}^{2}\right)}} .
$$


In Fig. 5.14 the lines represent the theoretical predictions for $v_{f}^{\text {theor }}$ given by Eq. (5.29). We see the perfect match with numerical data.

\subsection{Conclusion}

In this Chapter, we study two field theoretical models that contain field configurations that can be identified with particles. In the sine-Gordon theory we consider a topological soliton, and in the $\varphi^{4}$ theory a topological kink solution. Both these configurations represent solitary waves with the dispersion identical to that of a relativistic particle with finite mass. One can associate a conserved quantized topological charge $Q$ to each of these waves. In the sine-Gordon theory the "quantization" is $Q=2 \pi n$, with integer $n \in \mathbb{Z}$ due to the periodicity of the sine, and in the $\varphi^{4}$ theory the kink connects two minima of the potential so this charge can take the values $Q= \pm 2$. We study the behavior of the solutions that correspond to $Q=2 \pi$ (soliton) in the sine-Gordon theory and $Q=2$ (kink) in $\varphi^{4}$ theory, under the perturbations imposed by the re-scaling of the magnitude of the potential terms (the overall coupling constant). We study two situations: (i) a step-like spatial dependence of the coupling constant, which physically corresponds to the junction of two homogeneous systems, and (ii) a sudden change of the coupling constant in the whole space. The latter is comparable to a quench protocol studied in similar integrable one-dimensional systems [183].

For the spatially nonuniform setup we consider the soliton and the kink initially localized away from the junction and moving towards it with some initial velocity. Depending on the parameters of the system we observe either reflection or the transmission of the solitary wave after scattering on the boundary. In either case, the profile keeps its soliton/kink-like form with the additionally excited radiation modes. This physically manifests as small density ripples. Assuming that the contribution of the radiation to the total energy is negligible we use the energy conservation law to predict the critical velocity that separates reflective and transmitting regimes, and the value of the final velocity, when the solitary wave is transmitted. The total energy coincides with the energy of a relativistic particle, whose mass is different in different regions of space. We compare the theoretical prediction with a numerical solution and find perfect agreement for the values of the critical velocity, while the prediction for final velocity works reasonably well for the initial velocities substantially large than the critical one. The latter can be explained by the fact that the profile spends significant amount of time at the boundary rendering the expression for the total energy more complicated than for the relativistic particle. In particular, one could imagine, that the solitary wave moving exactly with the critical velocity would stick to the boundary, this way, creating a bound state. This situation is not realized in our numerical experiment. We try to create a bound state artificially considering initial profile as static solutions in each half-space con- 


\section{Solitary wave propagation in media with step-like inhomogeneities}

nected at the boundary. We observe that even in this case the profile starts moving as a solitary wave to the region with the small coupling constant transforming its potential energy into the finite velocity. Again, we predict the value of this velocity and find the perfect match with the numerical simulations. This confirms the robustness of the particle like structure of the soliton and the kink that does not depend on the exact form of the potential, i.e. it is identical for the sine-Gordon and the $\varphi^{4}$ models. The robustness follows from the topological protection and the fact that the ripples, or radiation modes always have a trivial charge, contrary to the nonlinear Schrödinger case $[184,185]$. We have also numerically investigated the penetration depth (the turning distance) for the solitary wave in the reflecting case. It turns out to be model dependent, i.e. it is different for the $\varphi^{4}$ model and the sine-Gordon model. We find that it can be fitted with exponential dependence as a function of the ratio of the coupling constants.

As a special case of spatially nonuniform setup we study the evolution of the moving wave between two junctions. We find a way to lock this wave with constant velocity in the finite region. Earlier, the motion of solitary waves in the Josephson junction that can be controlled or modulated by a magnetic field was discussed in $[182,187]$, both for experimental and theoretical setups. All the mentioned results are looking quite promising for implementing in telecommunications or rapid single flux quantum logic through a fluxon diode.

Finally, we studied the behavior of the moving solitary wave, when the value of the prefactor in front of the potential is quenched (suddenly changed). We numerically observe that the solitary wave remains solitary with the altered velocity. The energy is not conserved in the quench protocol, but since the system remains spatially homogeneous the total momentum is conserved. Again identifying the total momentum with the momentum of the relativistic particle, whose mass has a step-like time dependence we can predict the final value of the post-quench velocity. Comparing with the numerical simulation, we again find a perfect match. The key assumptions made here were the localized form of the profile and the small contribution of the radiation. Both these assumptions are possible due to topological nature of the initial profile and the fact that the conserved charge $Q$ is "quantized". This condition is not satisfied in the nonlinear Schrödinger case, so one cannot describe parameters of the quenched profile apart of the integrable cases $[183,184]$ or for the asymptotically large times [185]. Comparing this protocol with the previous one, for the spatial dependence, one could expect the naive self-quenching picture. Namely, it is tempting to describe a solitary wave that has tunneled into the region with a different coupling constant, as a result of quench in the total space. Indeed, the solitary wave is localized and the transition time is quite short. This picture, however, gives a wrong prediction for the final velocity it would derive from the conversation of energy and not momentum. Therefore, the transition time for the junction cannot be neglected.

We believe that the observed phenomena are robust and should be detect in 
any system with topologically protected excitations. i.e. excitations that have a quantized, conserved charge. Indeed, the value for critical and final velocities turned out to be identical for the sine-Gordon and the $\varphi^{4}$ models. Technically, this happens because the model dependent bare mass cancels in all calculations. So one would require more sophisticated observables to distinguish between the models. One such observable is the penetration depth of the slow solitons/kinks in the more dense media. The easiest, way to experimentally see these phenomena is perhaps, in nonuniform Josephson junctions described by the nonuniform sineGordon model [158, 159]. 


\section{Appendix}

\section{A Numerical method}

We numerically integrate the equation of motion given by Eq. (5.2) and determine the time evolution of any given initial field configuration. To do so, we are first of all forced by the finite computing power of any numerical code to restrict our attention to a finite interval of space and time. We thus consider only positions $-x_{\max } \leq x \leq x_{\max }$ and times $0 \leq t \leq t_{\max }$. Furthermore, we necessarily have to use a discrete grid within this continuous space-time interval. We choose a simple $(N+1)$ by $(M+1)$ rectangular grid, with step sizes $\Delta t=t_{\max } / N$ and $\Delta x=2 x_{\max } / M$. Points on this grid can be denoted by $(n, j)$ with $0 \leq n \leq N$ and $-M / 2 \leq j \leq M / 2$. These coincide with the continuous space-time coordinates at $t_{n}=n \Delta t$ and $x_{j}=j \Delta x$. To compute derivatives on the discrete grid, we employ a method of finite differences:

$$
\begin{array}{ll}
\left.\frac{\partial \varphi}{\partial t}\right|_{(t, x)=\left(t_{n}, x_{j}\right)} \approx \frac{\varphi_{j}^{n}-\varphi_{j}^{n-1}}{\Delta t},\left.\quad \frac{\partial^{2} \varphi}{\partial t^{2}}\right|_{(t, x)=\left(t_{n}, x_{j}\right)} \approx \frac{\varphi_{j}^{n+1}-2 \varphi_{j}^{n}+\varphi_{j}^{n-1}}{\Delta t^{2}}, \\
\left.\frac{\partial \varphi}{\partial x}\right|_{(t, x)=\left(t_{n}, x_{j}\right)} \approx \frac{\varphi_{j}^{n}-\varphi_{j-1}^{n}}{\Delta x},\left.\quad \frac{\partial^{2} \varphi}{\partial x^{2}}\right|_{(t, x)=\left(t_{n}, x_{j}\right)} \approx \frac{\varphi_{j+1}^{n}-2 \varphi_{j}^{n}+\varphi_{j-1}^{n}}{\Delta x^{2}} .
\end{array}
$$

The equation of motion for the particular case given by Eq. (5.2) for field values $\varphi_{j}^{n}$ on the discrete grid can now be written as:

$$
\varphi_{j}^{n+1}=2 \varphi_{j}^{n}-\varphi_{j}^{n-1}+\frac{\Delta t^{2}}{\Delta x^{2}}\left(\varphi_{j+1}^{n}-2 \varphi_{j}^{n}+\varphi_{j-1}^{n}\right)-\left.\zeta_{i} \Delta t^{2} \frac{d U}{d \varphi}\right|_{\varphi=\varphi_{j}^{n}},
$$

with $\zeta_{i}=\zeta_{1}$ for $-M / 2 \leq j<0$ and $\zeta_{i}=\zeta_{2}$ for $0 \leq j \leq M / 2$.

The initial conditions required to solve the complete dynamics then consist of the field configurations $\varphi_{j}^{0}$ and $\varphi_{j}^{1}$ at the initial two time slices. This can be thought of as specifying a position (amplitude) and velocity (rate of change) for all classical degrees of freedom (the field values) in the classical field theory. In general, the numerical simulation can be stable on the chosen rectangular grid as long as the temporal step size is smaller than the spatial one, i.e. $\Delta t<\Delta x$ [188].

A peculiarity of the finite differences used to compute spatial derivatives, is that they cannot be reliably computed at the edge of space because no information is available about the field values at $|j|=M / 2+1$. Physically, this means that any 
propagating wave in the field that reaches the edges of space will be reflected by the boundaries. To avoid seeing these unphysical reflections for the problem with open boundary conditions in the final simulated dynamics, the output of the code should only contain field values at positions $|j|<M / 2-N$. In this way, even the mistake made in the spatial derivative at time step $n=0$, or equivalently the wave that is reflected at the edge of space at $t=0$, has no time to propagate into the observed spatial interval.

To ensure that the numerical code functions correctly and that the inherent numerical error of the calculation does not get out of hand, several consistency checks can be considered. First of all, static solutions like vacua or the solitary wave should not change in time. Secondly, the total energy should be conserved in time, except for the energy flowing out of the window of observation defined by $|j|<M / 2-N$. This can be checked by computing the change in total energy, which should be close to zero:

$$
\begin{aligned}
\Delta E_{\text {tot }}\left(n^{\prime}\right) & =E\left(n=n^{\prime}\right)-E(n=0)-\sum_{n=0}^{n^{\prime}}\left[\frac{\partial \varphi}{\partial t} \frac{\partial \varphi}{\partial x}\right]_{j=-M / 2+N}^{M / 2-N} \\
\text { with } E(n) & =\sum_{j=-M / 2+N}^{M / 2-N}\left(\frac{1}{2}\left(\frac{\partial \varphi}{\partial t}\right)^{2}+\frac{1}{2}\left(\frac{\partial \varphi}{\partial x}\right)^{2}+\left(\zeta_{1} \theta(-x)+\zeta_{2} \theta(x)\right) U(\varphi)\right) .
\end{aligned}
$$

In these expressions, the partial derivatives should be interpreted in terms of the finite differences defined in Eq. (5.30). The conservation of energy $\left(\Delta E_{t o t}=0\right)$ can be checked at every time step in the simulation.

The final consistency check is to ensure that the field dynamics obtained in the numerical calculation does not change when decreasing the step sizes $\Delta t$ and $\Delta x$. The actual values of the step sizes can then be chosen empirically such that they ensure a desirable balance between having a manageable run time for the numerical code, and an adequate level of accuracy in the results. To quantify the latter, we should use both the independence of the result from the value of step sizes and the conservation of total energy. For simulations, it turns out that $\tau=0.01$ and $h=0.02$ are reasonable values for the choices of $t_{\max }=350$ and $x_{\max }=730$. The maximal detected deviation in energy is less than $2.0 \%$ for the sine-Gordon and $2.3 \%$ for the $\varphi^{4}$ models, respectively, which is reasonable because further decreasing of $\tau$ and $h$ can correct the numerical error in energy conservation, but has no affects on observing dynamics.

\section{B Systematic error for $v_{f}$}

We calculate an error for each value of $v_{f}$ given by Eq. (5.9) using systematic error 
5 Solitary wave propagation in media with step-like inhomogeneities

for indirect measurement as $\Delta v_{f}=\left[\left(x_{c}\left(t_{2}\right)-x_{c}\left(t_{1}\right)\right) \tau+\left(t_{2}-t_{1}\right) h\right] /\left[\left(t_{2}-t_{1}\right)^{2}\right]$, where $x_{c}$ is a center of solitary wave, $t_{1}$ and $t_{2}$ are some separated moments in time after interaction with a junction in the system, $\tau$ and $h$ are step sizes in the numerical scheme. In all presented results $\Delta v_{f}<2.2 \%$, and we decide to not put an error-bar on any corresponding plot. 


\section{Kinks and realistic impurity mod- els in the $\varphi^{4}$ theory}

We study the evolution of the kink and the kink-antikink pair in $(1+1)$-dimensional $\varphi^{4}$ field model in the presence of impurities. We start with the kink-impurity interactions. We consider the Dirac delta, the Gaussian, and the Lorentzian types of impurity, and we compare the observed dynamics between them. The excitation of impurity mode occurs, and its frequency fulfills the theoretical predictions. We continue with the kink-impurity-antikink interactions with the Dirac delta impurity. We discuss the observed phenomena such as oscillon, its bound state, and the resonances. We also study the behavior of the critical velocity as a function of impurity strength, and finally, we check the impurity mode for this type of initial conditions.

\subsection{Introduction}

The $\varphi^{4}$ theory is ubiquitous as a low-energy effective description of processes in all fields of physics ranging from cosmology to particle physics and condensed-matter theory, as we discussed already in Chapter 4. The topological defects, or kinks, in this theory describe stable, particle-like excitations. In chemistry and condensedmatter settings, actual impurities in the atomic lattice are an unavoidable fact of life, while in particle physics and cosmology variations in the potential or background metric act as impurities [31, 33, 35-38, 189].

First, we describe the interaction between kinks and various types of realistic impurity models simulated with the Dirac delta, the Gaussian, and the Lorentzian. We find that realistic impurities behave qualitatively like the well-studied, idealized delta function impurities, but that significant quantitative differences appear in both the characteristics of localized impurity modes and in the collision dynamics. We also identify a particular regime of kink-impurity interactions, in which kinks 
lose all of their kinetic energy upon colliding with an impurity.

Second, we study the influence of a localized impurity on the behavior of the kinkantikink pair for idealized delta function impurities. The dynamics is fruitful. We observe the capture of the kink-antikink pair, resonances and inelastic reflection, and excitation of the impurity modes for different values of impurity strength. Apart from that, we observe the birth of another quasi-long-lived solution, namely an oscillon-oscillon pair.

The outline of this Chapter is the following: in Section 6.3, we introduce the localized impurity in the scalar field. Then, we discuss the kink-impurity interactions. We start in Section 6.3.1 with Dirac delta limit of the Gaussian profile for an impurity, then in Section 6.3.2 we focus on the influence of impurity strength and width for the Gaussian profile, and in Section 6.3.3 we discuss the phenomenon of the impurity mode. We finish in Section 6.3.4 with discussions of the Lorentzian profile. Then we switch to kink-antikink interactions. In Section 6.4 we recall the well-known facts about kink-antikink collisions without an impurity. Then, in Section 6.5 we add an impurity in a system. In Section 6.5.1 we Dirac delta limit of the Gaussian profile for an impurity, later we study in detail the observed phenomena, namely an oscillon in Section 6.5.2, the critical velocity as a function of impurity strength in Section 6.5.3, and the impurity mode in Section 6.5.4. Finally, in Section 6.6 , we conclude.

\subsection{Impurity}

In the early days of kink research one [33] has already studied the kink-impurity collisions using idealized impurities, with a Dirac delta spatial profile. Here, we generalize this approach to include interactions between kinks and more realistic impurity models, based on Gaussian or Lorentzian spatial profiles. We find qualitative agreement with the physics of idealized defects described in [33], but significant quantitative effects of the impurity profile on the shape of the localized impurity mode, the scattering dynamics, and the possible long-time fate of kinks interacting with strong impurities.

We introduce a single inhomogeneity $\gamma(x)$ into the potential of Eq. (4.1) by writing $[33,35,36]$

$$
\frac{1}{4}\left(1-\varphi^{2}\right)^{2} \longrightarrow \frac{1}{4}\left(1-\varphi^{2}\right)^{2}\left(1-\epsilon \gamma\left(x-x_{0}\right)\right) .
$$

Here, $|\epsilon|<1$ and $x_{0}$ represent the strength and the center of a weak impurity. For $\epsilon=0$ the clean model is recovered, while $\epsilon<0$ and $\epsilon>0$ correspond to a repulsive barrier and an attractive potential well respectively. Including the impurity in a way of Eq. (6.1), the equation of motion becomes

$$
\varphi_{t t}-\varphi_{x x}+\left(\varphi^{3}-\varphi\right)\left(1-\epsilon \gamma\left(x-x_{0}\right)\right)=0 .
$$


We numerically solve Eq. (6.2) using a finite differences method (see Appendix 6.A for details). We consider the initial condition of a single kink $\varphi_{K}$, centered at $t=0$ at the position $a=6$, and moving with some initial velocity $v=v_{\text {in }}$ towards an impurity located at $x_{0}=0$. It is presented in Fig. 6.1 We explore the influence of the impurity profile $\gamma(x)$ on the kink-impurity scattering by considering three distinct types of impurities. The first is the idealized Dirac delta function originally proposed in [33]:

$$
\gamma\left(x-x_{0}\right) \rightarrow \delta\left(x-x_{0}\right)
$$

We then generalise to a more realistic Gaussian form for the impurity:

$$
\gamma\left(x-x_{0}\right) \rightarrow \frac{1}{\sigma \sqrt{2 \pi}} \exp \left[-\left(\frac{x-x_{0}}{\sigma \sqrt{2}}\right)^{2}\right] .
$$

Here, choosing $0<\sigma<1$ corresponds to the impurity width $\sigma$ being less than the width $l_{K}$ of the kink. We compare the results of the Gaussian impurity model with a final realistic ansatz, given by a Lorentzian profile:

$$
\gamma\left(x-x_{0}\right) \rightarrow \frac{1}{\left(x-x_{0}\right)^{2}+\alpha^{2}}
$$

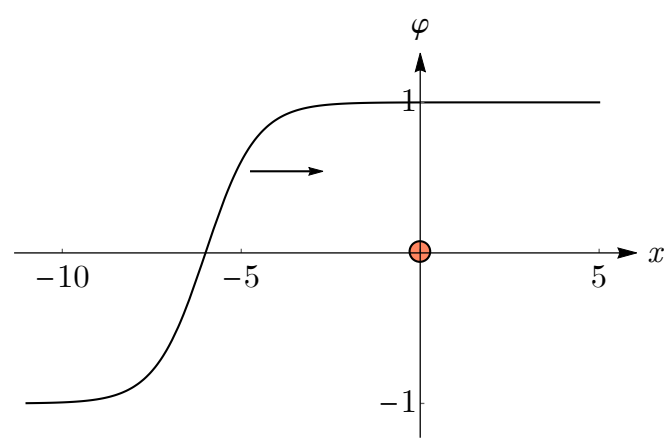

Figure 6.1: The kink at $t=0$ given by Eq. 4.16 with $a=6$. The arrow shows the directions of the wave motion. The orange circle shows the place of the impurity. 


\subsection{Kink-impurity interactions}

\subsubsection{Dirac delta limit of the Gaussian profile}

To establish a connection to the known result for the idealised Dirac delta impurity [33], we first consider a very narrow form of the Gaussian profile. We choose the height of the Gaussian at its center to coincide with the numerical value for the delta function height used in Ref. [33]. This results in a width $\sigma \simeq 0.016$ that is smaller than the lattice spacing in our numerical routine, in accordance with the Dirac delta limit.

For a repulsive impurity with $\epsilon=-0.5$, we reproduce the two types of processes known to occur as the value of $v_{i n}$ is varied [33]. For low initial velocities, such as $v_{i n}=0.4$ (shown in Fig. 6.2a), the kink is reflected by the impurity. For velocities above some critical value $v_{c r}$, the kink is transmitted, but its kinetic energy (velocity) is reduced in the process (part of it goes into excitations of small waves on top of the kink). This is shown in Fig. $6.2 \mathrm{~b}$ for the initial velocity $v_{i n}=0.8$.

In the case of an attractive impurity, with $\epsilon=+0.5$, three different types of known dynamics are reproduced [33]. At low initial velocities, such as $v_{\text {in }}=0.1$ (shown in Fig. 6.2c), the kink is captured by the impurity. That is, it ends up in a final state in which the kink center oscillates around the central position of the impurity. For high initial velocities on the other hand, like $v_{\text {in }}=0.5$ (shown in Fig. 6.2e), the kink is transmitted through the impurity entirely, loosing some kinetic energy in the process, and leaving behind a localized excitation centered at the impurity location. The localized impurity mode consists of small amplitude periodic oscillations of the field at $x=x_{0}$ around the vacuum value $\varphi_{v}^{(1)}$. The value of the initial velocity above which kinks are always transmitted through the impurity is called the critical velocity $v_{c r}$. For certain particular values of the initial velocity $v_{\text {in }}<v_{c r}$, such as $v_{i n}=0.137$ (shown in Fig. 6.2d), the kink is neither captured nor transmitted. Instead, it oscillates around the impurity center a finite number of times before leaving the impurity in the direction or opposite one it originally came from. This type of behavior is known as resonance, and can be understood in terms of the exchange of kinetic energy with internal excitations of the kink [33, 61].

For both attractive and repulsive impurity strengths, our results are in perfect agreement with those reported in Ref. [33], confirming that the idealized Dirac delta impurity gives a faithful approximation of the more realistic Gaussian profile in the limit of very low width. Further, we focus on its qualitative behavior as the strength and width of the impurity profile are varied.

Finally, we consider the limit of a very strong attractive impurity, with $\epsilon>1$. The resulting dynamics is presented in Fig. 6.3a - Fig. 6.3c. As the impurity strength increases, the captured kink oscillates around the impurity with ever lower amplitude, eventually getting stuck at the impurity position entirely. This regime, 
(a)

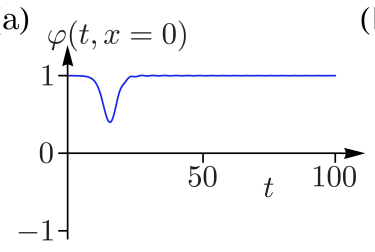

(b)

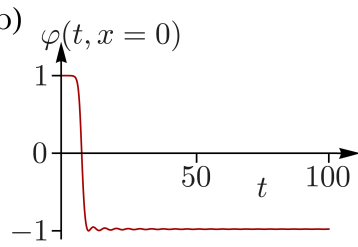

$$
\begin{aligned}
& \epsilon=-0.5 \\
& \stackrel{v_{c r}}{0} \approx 0.45 \quad v_{i n} \quad 1
\end{aligned}
$$

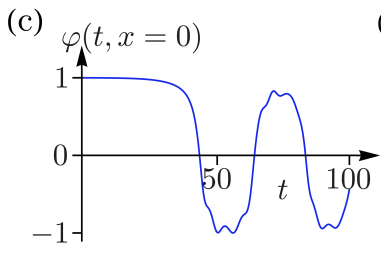

(d)

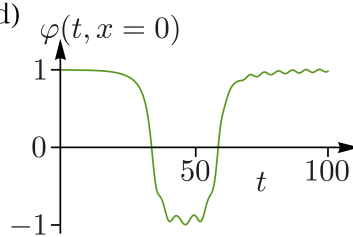

(e) $\varphi(t, x=0)$

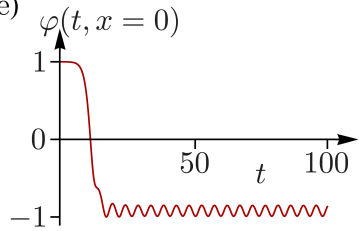

$$
\epsilon=0.5
$$

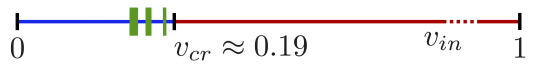

Figure 6.2: The value of the field $\varphi(t, x)$ at $x=0$ as a function of time, for a repulsive impurity with $\epsilon=-0.5$ and $\sigma \simeq 0.016$ (left of the dashed line) and for an attractive impurity with $\epsilon=+0.5$ and $\sigma \simeq 0.016$ (right of the dashed line). (a) For low initial velocity, $v_{i n}=0.4$, the kink is reflected by the impurity. (b) For high initial velocity, $v_{i n}=0.8$, the kink traverses the impurity, at the cost of loosing some kinetic energy. (c) For $v_{i n}=0.1$, the kink is captured by the attractive impurity. (d) For specific intermediate values such as $v_{i n}=0.137$, a resonance causes the kink to oscillate around the impurity before being released again. A few of the resonance windows are sketched along the bar of $v_{\text {in }}$ values at the bottom. (e) For high initial velocity, $v_{i n}=0.5$, the kink traverses the impurity, leaving behind an excited impurity mode, and loosing some kinetic energy.

in which the oscillation amplitude of a captured kink is smaller than its width, so that the field value at the impurity location never returns to either of its vacuum values $\varphi_{v}^{(1,2)}$, may be called a super-capture. Fig. 6.3d shows the dependence of the dominant oscillation frequency on impurity strength. 
6 Kinks and realistic impurity models in the $\varphi^{4}$ theory
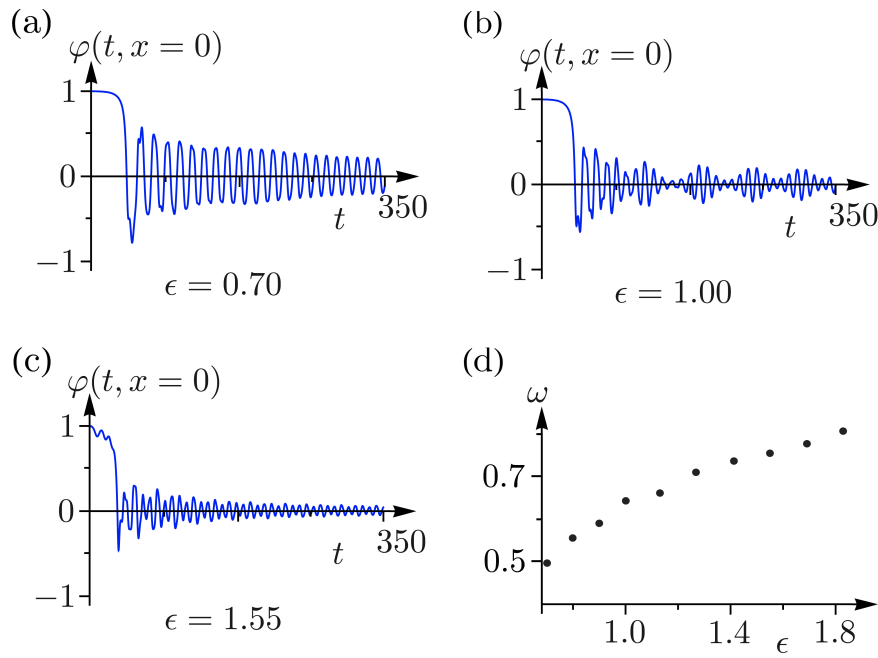

Figure 6.3: (a) - (c) The value of the field $\varphi(t, x)$ at $x=0$ as a function of time, for an attractive impurity with width $\sigma \simeq 0.016$, and fixed initial velocity of the kink $v_{i n}=0.1$. (a) For impurity strength $\epsilon=0.70$; (b) with $\epsilon=1.00$; and (c) for a very strongly attractive impurity with $\epsilon=1.55$. In all cases the kink is captured by the impurity and oscillates around the impurity center with an amplitude lower than the kink width. (d) The dominant frequency $\omega$ of oscillations of the captured kink as a function of impurity strength.

\subsubsection{Impurity strength and width}

For an ideal Dirac delta impurity, the value of $v_{c r}$ is determined entirely by the impurity strength $\epsilon$. For vanishing strength, the critical velocity tends to zero, while strong impurities cost a lot of kinetic energy to traverse, yielding a large $v_{c r}$. In the case of a Gaussian impurity profile, the width $\sigma$ of the impurity as well as its strength $\epsilon$ may be expected to play a role in determining the critical velocity, i.e. $v_{c r}=v_{c r}(\epsilon, \sigma)$.

The qualitative effects of the strength and impurity may be understood from the results in Table 6.1. The critical velocity increases in value upon either decreasing the width $\sigma$ for fixed values of the strength $|\epsilon|$, or upon increasing $|\epsilon|$ for fixed values of $\sigma$. These trends can be understood by comparing Eq. (6.2) and Eq. (6.4), which show that the height of the impurity potential at $x_{0}$ is proportional to the ratio $\epsilon / \sigma$. 


\begin{tabular}{cccc}
$\epsilon$ & $\sigma$ & $v_{i n}$ & fate of kink \\
\hline 0.3 & 0.5 & 0.02 & transmitted \\
0.3 & 0.3 & 0.02 & captured \\
0.3 & 0.3 & 0.05 & transmitted \\
0.3 & 0.1 & 0.05 & captured \\
& & & \\
-0.6 & 0.5 & 0.47 & transmitted \\
-0.6 & 0.3 & 0.47 & reflected \\
-0.6 & 0.3 & 0.50 & transmitted \\
-0.6 & 0.1 & 0.50 & reflected
\end{tabular}

\begin{tabular}{cccc}
$\epsilon$ & $\sigma$ & $v_{\text {in }}$ & fate of kink \\
\hline 0.4 & 0.3 & 0.10 & transmitted \\
0.6 & 0.3 & 0.10 & captured \\
0.6 & 0.3 & 0.28 & transmitted \\
0.8 & 0.3 & 0.28 & captured \\
& & & \\
-0.1 & 0.3 & 0.3 & transmitted \\
-0.4 & 0.3 & 0.3 & reflected \\
-0.4 & 0.3 & 0.5 & transmitted \\
-0.8 & 0.3 & 0.5 & reflected
\end{tabular}

Table 6.1: (left) The effect of varying impurity width on the kink-impurity interaction, for fixed value of the impurity strength. Larger values of $\sigma$ are seen to yield lower values of $v_{c r}$. (right) The effect of varying impurity strength, for fixed value of the impurity width. Larger values of $|\epsilon|$ are seen to yield higher values of $v_{c r}$.

To further confirm that the interaction of kinks with a wide impurity differs only quantitatively from that with a narrow impurity, we confirm that resonances still occur for realistic impurity widths. For the values $\epsilon=0.5$ and $\sigma \simeq 0.016$ there is a resonance around $v_{i n}=0.137$. Upon increasing the impurity width to $\sigma=0.1$, we instead find a resonance at $v_{i n}=0.150$. At $\epsilon=0.6$ and $\sigma=0.3$ we observe a resonance at the same value of $v_{i n}$. The qualitative behavior of having resonance windows below $v_{c r}$ for attractive impurities thus survives also for more realistic impurity profiles.

\subsubsection{The impurity mode}

When a kink traverses an attractive impurity it leaves behind an oscillating mode, localized at the site of the impurity (see Fig. 6.4a). This mode oscillates at $x=x_{0}$ with near-constant amplitude and frequency, as shown in Fig. 6.4b. It is a quasilong-lived mode, analogous to the wobbling kink [178, 179], and bion solutions found in kink-antikink collisions [59] (see Section 6.4).

For the case of the idealised Dirac delta impurity, the approximate shape of the impurity mode profile can be found analytically [33]. Since the mode is localized at the impurity site and excited by the passing of a kink, we can write it as a small deviation $\delta \varphi$ from the vacuum solutions $\varphi_{v}^{(1,2)}$. Further, we write $\varphi_{v}^{(1)}$ because this particular vacuum is related to the discussions above. Substituting $\varphi(t, x)=$ $\varphi_{v}^{(1)}+\delta \varphi(t, x)$ in Eq. (6.2) and Eq. (6.3) and keeping only terms up to linear order 
6 Kinks and realistic impurity models in the $\varphi^{4}$ theory

(a)

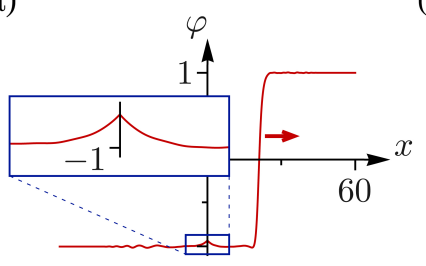

(b)

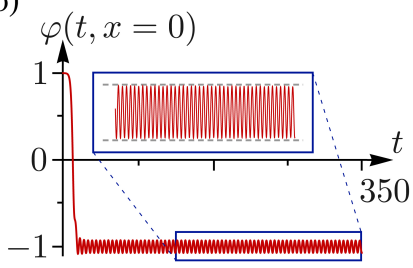

Figure 6.4: (a) The spatial profile of the impurity mode, excited by a kink traversing the impurity site $x=x_{0}=0$ and travelling to the right. (b) The value of the field $\varphi(t, x)$ at $x=x_{0}=0$ as a function of time, for an attractive impurity with width $\sigma=0.5$ and strength $\epsilon=0.5$. An impurity mode is excited by a kink with initial velocity $v_{i n}=0.5$ passing $x=x_{0}$ at approximately $t \approx 20$. The horizontal dashed lines in the inset are guides to the eye highlighting the quasi-long-lived character of the impurity mode.

(a)

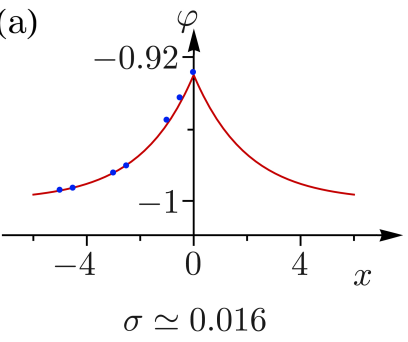

(b)

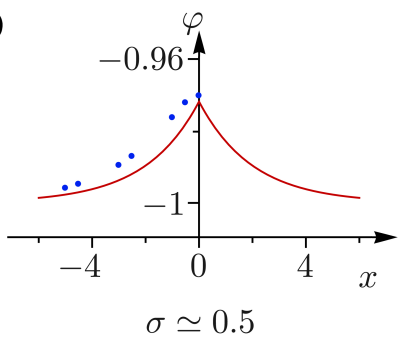

Figure 6.5: (a) Comparison of the numerically obtained values for the field $\varphi(t, x)$ after a kink traversed the impurity site (blue dots), with the analytical prediction of Eq. (6.7) (red line). The impurity potential used is a narrow Gaussian with strength $\epsilon=0.5$ and width $\sigma \simeq 0.016$, while the incoming kink had initial velocity $v_{i n}=0.5$. (b) The same comparison using a wide Gaussian impurity potential with strength $\epsilon=0.5$ and width $\sigma \simeq 0.5$.

in $\delta \varphi$ yields the equation of motion for the impurity mode:

$$
\delta \varphi_{t t}-\delta \varphi_{x x}+2\left(1-\epsilon \delta\left(x-x_{0}\right)\right) \delta \varphi=0 .
$$

Using the ansatz that the impurity mode oscillates with a fixed spatial profile and 
constant frequency, $\delta \varphi(t, x) \propto \Re \chi(x) e^{-i \Omega t}$, it is found to obey [33]:

$$
\delta \varphi(t, x) \propto \Re \exp (-\epsilon|x|) e^{-i \Omega t}, \quad \Omega^{2}=2-\epsilon^{2} .
$$

The numerically obtained field configuration in Fig. 6.5a indicates that for a very narrow impurity potential, $\epsilon=0.5$ and $\sigma \simeq 0.016$, an incoming kink with $v_{\text {in }}=0.5$ excites an impurity mode whose shape closely matches the prediction of Eq. (6.7). The numerical results (see Appendix 6.B) in Fig. 6.5b on the the other hand, show that for wider impurity potential, $\epsilon=0.5$ and $\sigma \simeq 0.5$, the analytic solution no longer gives an accurate prediction for the impurity mode profile. The spatially smooth impurity potential in this case does not allow for any discontinuities in the field configuration or its derivatives, forcing the impurity mode profile to remain smooth around the impurity location.

Besides the spatial profile of the impurity mode, its amplitude $A$ and frequency $\tilde{\Omega}$ may also vary with impurity width, and deviate from the analytic prediction of Eq. (6.7). For narrow impurity potential $(\sigma \simeq 0.016)$ we find $\langle\tilde{\Omega}\rangle \simeq 1.382$ for $\epsilon=0.3$ and $\langle\tilde{\Omega}\rangle \simeq 1.323$ for $\epsilon=0.5$, both in excellent agreement with the analytic prediction of Eq. (6.7). For wider impurities, the approximations underlying the analytic solution break down, and we numerically find the behavior displayed in Fig. 6.6.

The frequencies in Fig. 6.6b do not vary much with the initial velocities. Taking the average over all points shown yields a value of $\langle\tilde{\Omega}\rangle \simeq 1.387$ for $\epsilon=\sigma=0.3$, and $\langle\tilde{\Omega}\rangle \simeq 1.355$ for $\epsilon=\sigma=0.5$. The decrease of the frequency with increasing impurity strength indicated in Fig. 6.6d is in line with the analytic prediction of Eq. (6.7) for ideal Dirac delta impurities, but is now seen to also depend on the width $\sigma$ of the more realistic Gaussian profile. The frequency increases with increasing width, as shown in Fig. 6.6f, in agreement with the fact that increased width in Eq. (6.4) implies a lower value of the impurity potential at $x=x_{0}$.

\subsubsection{Lorentzian profile}

To see whether the qualitative effects of a wide impurity profile, rather than an idealised Dirac delta form, are generic to more realistic impurity models, we next compare the results of the Gaussian case to interactions between a kink and a Lorentzian impurity. We repeat the analysis of Section 6.3.1 using the impurity profile of Eq. (6.5), with $\epsilon= \pm 0.5$. To ensure that the peak height at $x_{0}=0$ matches that of the Gaussian considered before, we take $\alpha=0.2$.

For the repulsive Lorentzian impurity, we find that the kink is reflected for all initial velocities up to $v_{i n}=0.9$. However, at $v_{i n}=0.99$ the kink traverses the impurity, showing that a critical velocity exists in the range $0.90<v_{\text {in }}<0.99$. Reversing the sign of $\epsilon$ and considering an attractive Lorentzian impurity, we ob- 
6 Kinks and realistic impurity models in the $\varphi^{4}$ theory

(a)

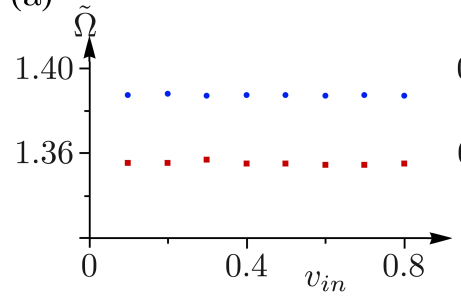

- $\epsilon=0.3 \sigma=0.3$

(c)

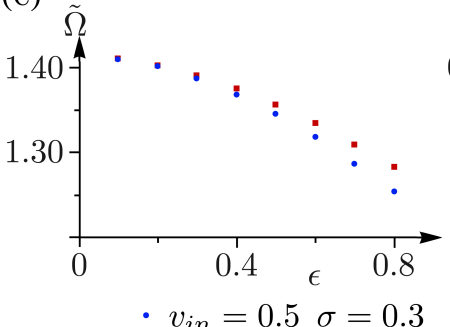

- $v_{\text {in }}=0.5 \quad \sigma=0.3$

(e)

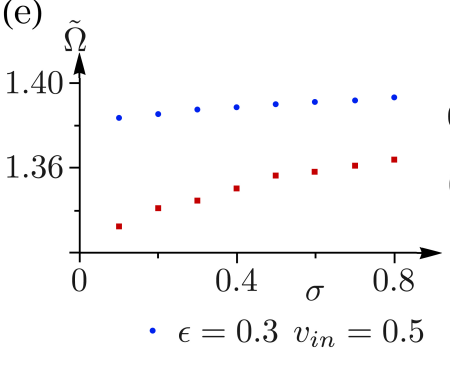

(b)

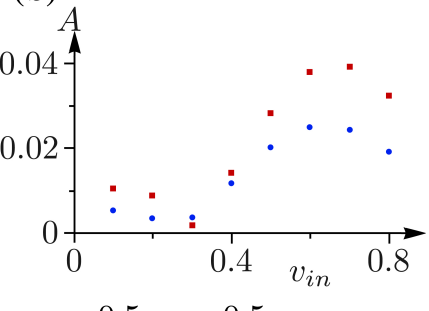

(d)

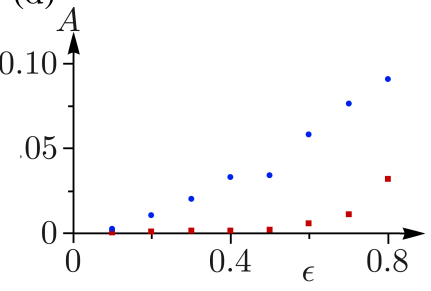

$v_{i n}=0.3 \quad \sigma=0.5$

(f)

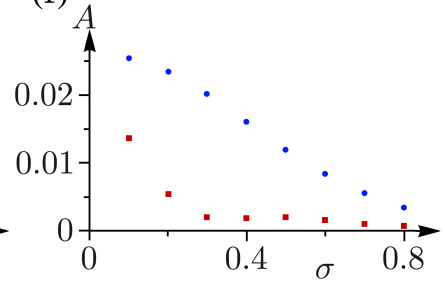

$\epsilon=0.5 v_{\text {in }}=0.3$

Figure 6.6: (a) The impurity mode frequency $\tilde{\Omega}$ and (b) its amplitude $A$, as a function of the initial kink velocity $v_{i n}$, for fixed values of impurity strength and width. (c) The frequency and (d) amplitude as a function of impurity strength $\epsilon$, keeping the initial velocity and impurity width constant. (d) Frequency and (e) amplitude as a function of the the impurity width $\sigma$, for fixed impurity strength and initial velocity.

serve that for initial velocities below $v_{i n}=0.8$, the kink is always captured by the impurity.

Although the capturing and reflecting of kinks is qualitatively similar to the behavior in the presence of a Gaussian impurity, the values of the critical velocities are much higher for a Lorentzian profile than for a Gaussian with the same maximum 
value. We therefore also compare Lorentzian and Gaussian profiles with the same integrated strength, by using the scaled function:

$$
\frac{\alpha / \pi}{\left(x-x_{0}\right)^{2}+\alpha^{2}} \text {. }
$$

We again take $\epsilon= \pm 0.5$ and $\alpha=0.2$.

In this case, the kink is reflected by a repulsive impurity for initial velocities up to $v_{i n}=0.4$, and traverses the impurity site for velocities $v_{i n}=0.5$ and above. Likewise, the attractive impurity captures the kink at $v_{i n}=0.01$, while it is transmitted for initial velocities above $v_{\text {in }}=0.1$.

These results indicate a qualitative agreement between the dynamics of kinkimpurity interactions for Gaussian and Lorentzian impurity profiles. We leave for future study, e.g., looking for resonances around the Lorentzian impurity, investigating the precise dependence of the critical velocity on the parameter $\alpha$, and so on.

\subsection{Kink-antikink interactions}

In [42] it was pointed out that the dynamical properties of the protein chains may be defined by the corresponding kink dynamics. One of the most well-known examples of kink dynamics is kink-antikink interaction [60, 61, 64].

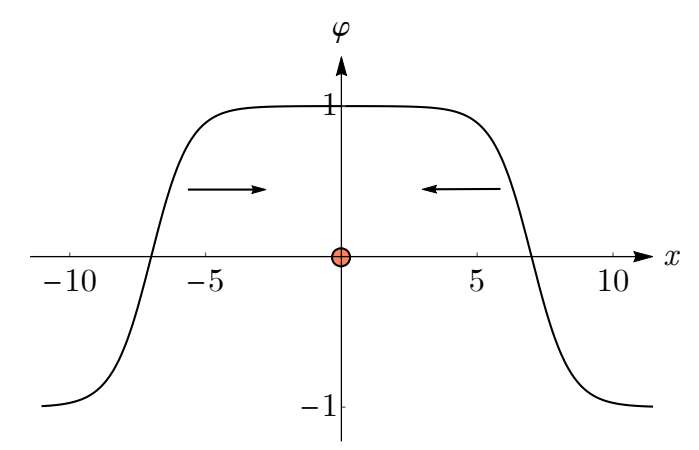

Figure 6.7: The kink-antikink pair at $t=0$ given by Eq. 6.9 with $a=7$. The arrows show the directions of the wave motion. The orange circle shows the place of the impurity, for the case described in Sec. 6.5.

To unveil the dynamics of the kink-antikink pair one should numerically solve 
6 Kinks and realistic impurity models in the $\varphi^{4}$ theory

Eq. (4.14) with the initial condition

$$
\varphi_{K \bar{K}}(t, x)=\tanh \left(\frac{x+a-v_{i n} t}{\sqrt{2\left(1-v_{i n}^{2}\right)}}\right)-\tanh \left(\frac{x-a+v_{i n} t}{\sqrt{2\left(1-v_{i n}^{2}\right)}}\right)-1,
$$

namely the kink $\varphi_{K}$ located at point $x=-a$ and the antikink $\varphi_{\bar{K}}$ located at $x=a$ with $2 l_{K} \ll 2 a$ at $t=0$, moving towards each other with some initial velocity $0<v_{\text {in }}<1$. This configuration is presented in Fig. 6.7. One observes three qualitatively different scattering regimes depending on $v_{i n}$ that are separated by a critical velocity $v_{c r}$ such that

$\diamond$ for $v_{i n}<v_{c r}$ there is so-called bion or breather (Fig. 6.8a), i.e. a localized at $x=0$ quasi-long-lived oscillating around the vacuum $\varphi_{v}^{(1)}$ configuration $[61,178]$ with a large amplitude that may be simulated in terms of the collective coordinate approximation (see Appendix 6.C) or a special type of ansatz containing Eq. (4.15) and Eq. (4.17) (see Appendix 6.D);

$\diamond$ for some special values of $v_{i n}<v_{c r}$ there are resonances [61] (Fig. 6.8b), when the kink and antikink can break the bound state after a finite amount of impacts; this phenomenon may be explained by redistribution of kinetic and potential energies [61];

$\diamond$ for $v_{i n} \geq v_{c r}$ there is an inelastic reflection (Fig. 6.8c) with a small loss of kinetic energy, i.e. after first impact the kink and the antikink scatter to infinity in opposite directions relative to the original one.

The critical initial velocity $v_{c r}$ was determined [61] as $v_{c r} \approx 0.2598$ for $a=7$ (it does not depend on $a$ if $2 l_{K} \ll 2 a$ ) such that for $v_{i n} \geq v_{c r}$ there were no observed collisions during $t=300$ after the first collision.

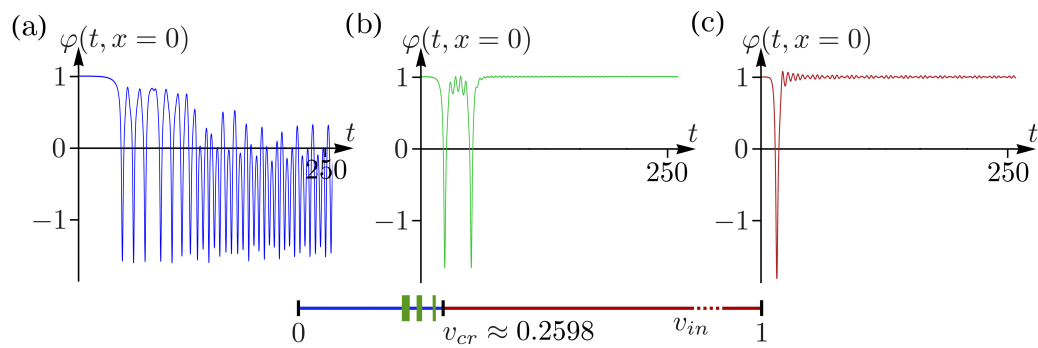

Figure 6.8: Three types of the kink-antikink collisions in absence of the impurity: the bion for $v_{i n}=0.1$, the resonance for $v_{i n}=0.2384$, and inelastic reflection for $v_{i n}=0.5$. For all plots we use $a=7$ in Eq. (6.9). 


\subsection{Kink-impurity-antikink interactions}

The next possible research step in to include one point-like impurity in between the kink-antikink pair $\varphi_{K \bar{K}}$ given by Eq. (6.9). The initial condition for this case is presented in Fig. 6.7. We introduce the inhomogeneity in the system in the same way as it was discussed in Section 6.3 Here, we focus only on the behavior when the impurity is present. Thus, we take only the Dirac delta limit of the Gaussian profile of impurity, i.e. further we discuss only $\sigma \simeq 0.016$ (for the same reasons as we discussed in Section 6.3). Below, we avoid discussions of high values of $\epsilon>0$ because we could not reach the required time scale for the chosen steps, however, in general, it is possible after choosing different steps while focusing on the implementation of the law of energy conservation.

\subsubsection{Dirac delta limit of the Gaussian profile}

In order to unveil the general influence of localized disorder on the system dynamics, we fix $|\epsilon|=0.2$.

In the case of a repulsive impurity we observe three types of processes. For $v_{\text {in }}<v_{\text {cr }}$ after the first collisions, the kink-antikink pair forms a special bound state of two oscillons (see Fig. 6.9a and Fig. 6.10) or a moving oscillon-oscillon pair (see Fig. 6.11). An oscillon is another quasi-long-lived bound state of the $\varphi^{4}$ model that has a shape of a Gaussian [190]. More details can be found in Section 6.5.2. For $v_{i n} \geq v_{c r}$ the kink and the antikink leave each other to infinity after one collision. For some special values of $v_{i n}<v_{c r}$ we observe a resonance phenomena (similar to Fig. 6.8b). Note that unlike here, for kink-impurity interactions described in Section 6.3 the resonance phenomena are observed only for attractive impurities.
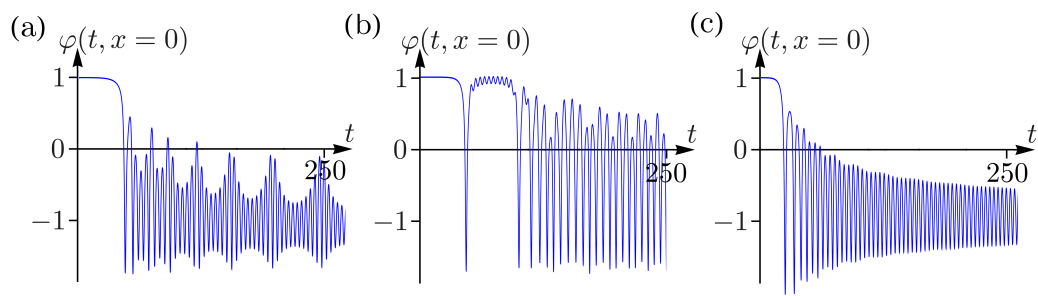

Figure 6.9: Different types of bound states for subcritical initial velocities in the kink-impurity-antikink collisions. The transition from (a) oscillonoscillon bound state $\left(\epsilon=-0.2, v_{i n}=0.1\right)$ through (b) a quasi-breather $\left(\epsilon=0.15, v_{i n}=0.15\right)$ to (c) a decay into a vacuum state $(\epsilon=0.4$, $\left.v_{\text {in }}=0.2\right)$. 
6 Kinks and realistic impurity models in the $\varphi^{4}$ theory

(a)

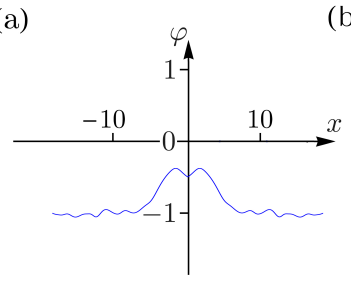

(b)

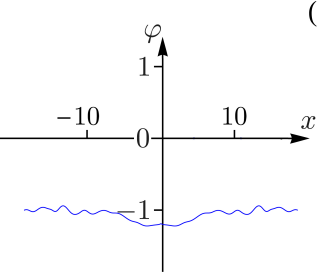

(c)

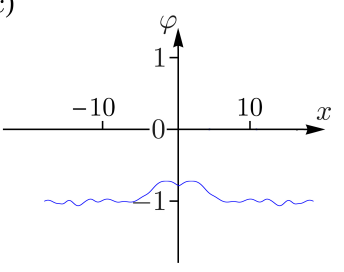

Figure 6.10: Oscillon-oscillon bound state for $\epsilon=-0.2$ and $v_{\text {in }}=0.1$. The profiles of the field $\varphi(x)$ for some chosen values of $t$.

(a)

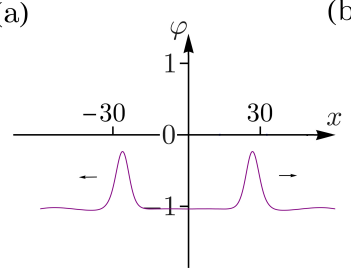

(b)

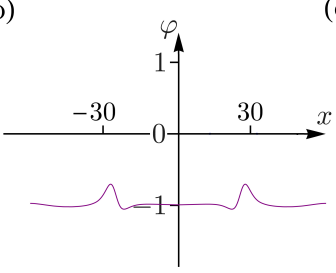

(c)

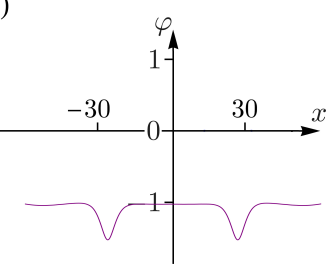

Figure 6.11: Moving oscillon-oscillon pair for $\epsilon=-0.2$ and $v_{\text {in }}=0.3$. The profiles of the field $\varphi(x)$ for some chosen values of $t,(\mathrm{a})-(\mathrm{c})$ correspond to approximately half a period of oscillations. The arrows on (a) represent the direction of the oscillon motion.

For an attractive impurity the dynamics is as follows. For $v_{i n}<v_{c r}$ after the first collision a bion is formed, however, due to presence of an attractive impurity it is no longer a quasi-long-lived solution and it decays to the vacuum $\varphi_{v}^{(1)}$ faster (see Fig. $6.9 \mathrm{~b}$ and Fig. $6.9 \mathrm{c}$ ). For $v_{i n} \geq v_{c r}$ the kink and the antikink leave to infinity leaving behind an excitation of an impurity mode at $x=x_{0}$ (similar to Fig. 6.4, a detailed research is presented in Section 6.5.4). Again, for some special values of $v_{i n}<v_{c r}$ resonance phenomena occur (similar to Fig. $6.8 \mathrm{~b}$ ).

We observe the resonances for other values of $\epsilon$ as well, e.g., for $\epsilon=-0.8\left(v_{i n}=\right.$ $\left.0.771, v_{i n}=0.772\right), \epsilon=-0.4\left(v_{i n}=0.540, v_{i n}=0.555, v_{i n}=0.557\right), \epsilon=0.05$ $\left(v_{i n}=0.210\right)$, and $\epsilon=0.15\left(v_{i n}=0.153\right)$.

\subsubsection{Oscillon}

As we mentioned in Section 6.5.1 for $\epsilon<0$ and $v_{i n}<v_{c r}$, we observe either a bound state of two oscillons (Fig. 6.10) or a moving oscillon-oscillon pair (Fig. 6.11).

An oscillon $\varphi_{O}$ is another quasi-long-lived bound state of the $\varphi^{4}$ model together with the breather that oscillates around one of the vacua $\varphi_{v}^{(1)}$ or $\varphi_{v}^{(2)}$. It has a 
Gaussian shape in peak [190]

$$
\varphi_{O}(x)=A_{O} \exp \left[-\frac{(x-B)^{2}}{C^{2}}\right],
$$

where $A_{O}$ is an amplitude, $B$ is a center, and $C$ is a width. In contrast to the breather, the oscillon is characterized by a small amplitude. In [190] was shown for $(1+3)$ dimensions that for

$$
2.90 \leq C \leq 3.54
$$

with $C$ as a radius of a bubble that relaxes in an oscillon, one obtains the longestlived oscillon. Thus, one can expect if the oscillon occurs in the system, the most likely form of Eq. (6.10) with constraint given by Eq. (6.11).

In order to ensure that the observed phenomena presented in Fig. 6.10 and Fig. 6.11 are exactly related to the oscillons, we take the field configurations $\varphi(t, x)$ for some $t$, when we observe the maximum peak, and fit them with Eq. (6.10). We do this for two particular cases of kink-impurity-antikink collisions, namely for $\epsilon=-0.2, v_{\text {in }}=0.30$ and $\epsilon=-0.3, v_{\text {in }}=0.43$, for which we observe moving oscillon-oscillon pair. We take different $\epsilon$ and $v_{\text {in }}$ to check the dependencies of an oscillon on these parameters.

For $\epsilon=-0.2$ and $v_{\text {in }}=0.30$ the numerically obtained field configuration fits Eq. (6.10) with $A_{O}=0.58$ and $C=3.07$ (mean values). For $\epsilon=-0.4$ and $v_{\text {in }}=0.43$ we obtain $A_{O}=0.51$ and $C=3.02$ (mean values). The difference between these results is $1.6 \%$ for $C$, and they both obey the prediction from Eq. (6.11). We obtain a similar period of oscillations almost equals to five that yields $\omega_{O} \simeq 1.26$. However, the final velocity of the separating oscillons is different, i.e. $v_{f}=0.09$ and $v_{f}=0.16$ for these two cases, correspondingly. We can conclude that the initial parameters of kink-impurity-antikink interactions $\left(\epsilon\right.$ and $\left.v_{i n}\right)$ mostly influence the amplitude of the oscillons and the final velocity of oscillon-oscillon pair, and barely affects the width of the oscillon and its period. We overlay the numerically obtained profile of an oscillon-oscillon pair for some fixed $t$ with the oscillon ansatz given by Eq. (6.11), with $A$ and $C$ obtained from a fit, in Fig. 6.12a and see a perfect match.

As we mentioned above, apart from moving oscillon-oscillon pairs for $\epsilon<0$ and $v_{i n}<v_{c r}$ we see the bound state of two oscillons. To ensure that the observed bound state is nothing else but the bound state of two oscillons, we overlay the numerically obtained profile of this configuration for some fixed $t$ with the oscillon ansatz given by Eq. (6.11), with $A$ and $C$ obtained from a fit, in Fig. $6.12 \mathrm{~b}$ and see a perfect match.

The presence of an oscillon-oscillon bound state is a sign that a moving oscillonoscillon pair is a resonance in comparison with a bound state of two of them, that occurs due to an impurity in the system. These resonances are similar to the same in kink-antikink collisions (see Section 6.4) in the sense that the oscillons can form 
(a)

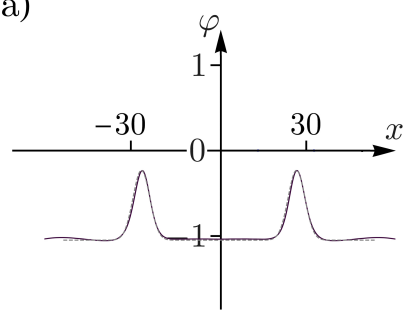

(b)

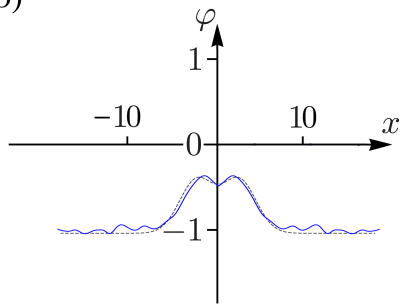

Figure 6.12: The profile of the field $\varphi$ for some chosen $t$ (solid line) and overlap with oscillon ansatz given by Eq. (6.11) (gray dashed line) with $A_{O}=$ 0.58 and $C=3.07$ and some $B$. (a) Oscillon-oscillon pair, $\epsilon=-0.2$ and $v_{i n}=0.30$. (b) Oscillon-oscillon bound state, $\epsilon=-0.2$ and $v_{\text {in }}=0.1$.

a moving oscillon-oscillon pair instead of a bound state after a finite amount of impacts (but not mandatory after two).

Earlier, the oscillons were found in the sinh-deformed [72, 73] $(1+1)$-dimensional $\varphi^{4}$ model without an impurity after kink-antikink collisions [74]. In this work, the authors observe a similar type of behavior, namely a bound state of two oscillons and so-called oscillons' escape windows, when two oscillons are moving from each other as a post-collision phenomenon. In [74] the resonance was explained by the resonant energy exchange between the translational and the vibrational modes of the deformed kink and the deformed antikink. To prove that the authors compare the frequency of the oscillon $\omega_{O}^{\text {def }} \simeq 1.88$ to the vibrational mode of the deformed kink $\omega_{1}^{\text {def }} \simeq 1.89$. If we compare our result for the oscillon frequency of $\omega_{O} \simeq 1.26$ to the vibrational mode of the kink $\omega_{1}=\sqrt{3 / 2} \simeq 1.22$ (see Eq. (4.27) in Appendix 4.A in Chapter 4) we can also see the frequency matching. This allows us to conclude that the formation of oscillon pairs might be explained by the resonant energy exchange between the translational and the vibrational modes of the kink in the kink-impurity-antikink system. However, we describe in Section 6.5.1 that apart from this phenomenon, we observe the regular resonance when the kink and the anitkink are leaving each other after a finite amount of impacts as the kink and the antikink but not as two oscillons. The difference in resonance mechanisms between these two processes can be interesting for future research.

\subsubsection{The critical velocity}

In Section 6.5.1 we describe different phenomena in kink-impurity-antikink collisions. They are defined by the critical velocity that is different for each value of 
impurity strength. One could guess that the stronger (by absolute value) the impurity becomes, the higher is critical velocity. However, this does not happen for kink-impurity-antikink interactions.

We determine the critical velocity for $-0.9<\epsilon \leq 0$ with step 0.1 and $0<\epsilon \leq 0.4$ with step 0.05. The dependence of $v_{c r}$ on $\epsilon$ is presented in Fig. 6.13. We notice that the critical velocity $v_{c r}$ decreases for $\epsilon \leq 0.2$, while afterwards it starts to increase. In order to understand it, we study more carefully what happens to the system for $v_{i n}<v_{c r}$ for different values of $\epsilon$. In Fig. 6.9 we show the transition from a bound state of two oscillons to the vanishing of a bound state of the kink and the antikink by going from a high negative value of $\epsilon$ to a high positive value of $\epsilon$. We conclude that the kinetic energy of the initial kink-antikink pair goes into either the formation of oscillons or into the faster vanishing of the breather into the vacuum $\varphi_{v}^{(1)}$.

The decay of a breather into a vacuum $\varphi_{v}^{(1)}$ might be accompanied by the excitation of an impurity mode (see Sections 6.3.1 and 6.3.3). An indication of this is that, e.g., for $\epsilon=0.5$ and $v_{i n}=0.4$ we observe a stable oscillation with frequency $\tilde{\Omega} \simeq 1$.30. For this value of $\epsilon$ a theoretical prediction is given by Eq. (6.7) for the impurity mode frequency $\Omega \simeq 1.32$. Nevertheless, we observe the decay of the inpeak amplitude of the oscillation, for $t=350$ it is $A=0.3$, which is higher than regular impurity mode amplitude. This decay can be a sign that we do not reach a moment of time (due to numerical restriction, see Appendix 6.A) when the breather has not vanished completely, and that only the impurity mode stays. This question can be an interesting topic for future detailed research.

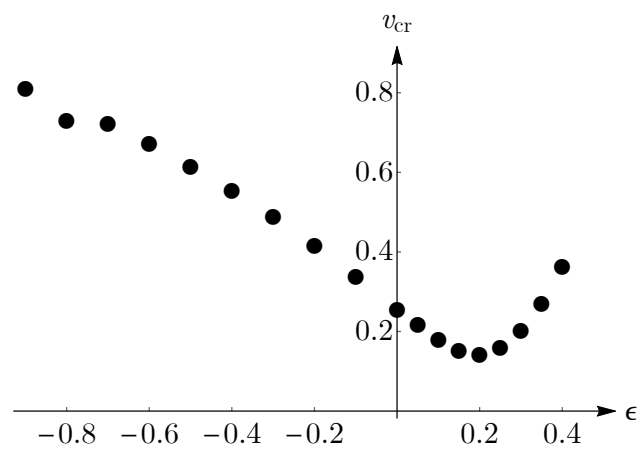

Figure 6.13: The dependence of the critical velocity $v_{c r}$ on impurity strength $\epsilon$. 


\subsubsection{The impurity mode}

When the kink and the antikink collide with $v_{i n}>v_{c r}$ with an attractive impurity in between, after the first collision they go away from each other, and leave behind an oscillating mode, localized at the site of the impurity $x=x_{0}$ (exactly the same as in the Fig. 6.4). This effect one can observe for interactions of one kink with an impurity that we describe in Section 6.3.1. We use the numerically obtained impurity mode that is characterized by frequency $\tilde{\Omega}$ and the peak amplitude $A$, to check a coincidence with prediction given by Eq. (6.7). The procedure is the same at the one described in Section 6.3.3. The results that are presented in Fig. 6.14 perfectly match the predictions. From the data in Fig. 6.14 a we obtain that $\langle\tilde{\Omega}\rangle \simeq$ 1.41 (maximal deviation is $0.45 \%$, for $\epsilon=0.1$ ) and $\langle\tilde{\Omega}\rangle \simeq 1.38$ (maximal deviation is $1.10 \%$, for $\epsilon=0.3$, which means that the frequency of the impurity $\tilde{\Omega}$ almost does not depend on the initial velocity of the incoming waves. Fig. $6.14 \mathrm{c}$ also shows a perfect match between the numerical data and the theoretical predictions for $\tilde{\Omega}$. The maximum observed deviation for $\epsilon=0.3$ is $0.35 \%$.
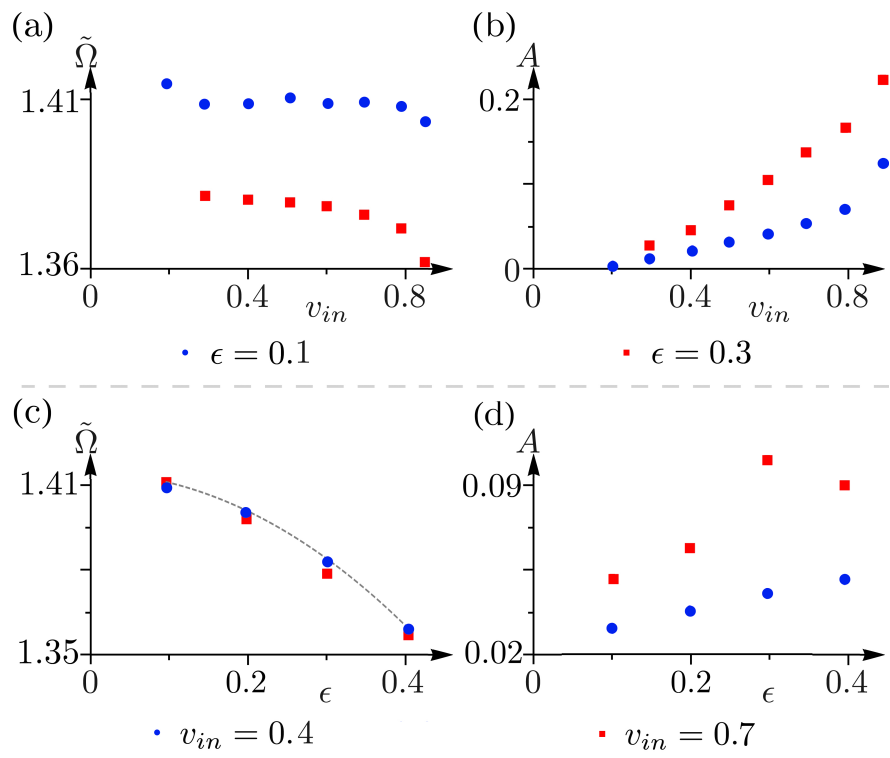

Figure 6.14: (a) The impurity mode frequency $\tilde{\Omega}$ and (b) its amplitude $A$, as a function of the initial kink and antikink velocity $v_{i n}$, for fixed values of impurity strength. (c) The frequency and (d) amplitude as a function of the impurity strength $\epsilon$, keeping the initial velocity constant. 


\subsection{Conclusion}

In this Chapter, we have shown that the behavior of kink-impurity interactions in $(1+1)$-dimensional $\varphi^{4}$ theory is qualitatively the same for idealised Dirac delta impurity profiles and more realistic Gaussian or Lorentzian shapes. In all cases, repulsive impurities can either reflect or transmit an incoming kink, depending on its initial velocity. Attractive impurities on the other hand either capture the kink, release after a few oscillations in a resonant process, or transmit it immediately. In the latter case, an impurity mode is excited at the impurity location by the passing kink. We also observe that a kink impinging on particularly strong impurities may lead to an extreme form of capture, in which the kink looses all of its kinetic energy and remains localized at the impurity site.

The Gaussian profile reduces to precisely a Dirac delta impurity in the limit of vanishing width, and all observed properties of the kink-impurity interaction agree with the known results for ideal impurities in that limit. For wider impurity profiles, the results differ quantitatively from the ideal case. The amplitude and frequency of the impurity mode obtain a dependence on the impurity width, the values of critical velocities are affected, and the kink velocity at which resonances appear is altered. Similar quantitative effects are observed for interactions between a kink and a Lorentzian impurity.

Then, we have shown that in kink-impurity-antikink interactions an impurity mode is presented for an attractive impurity. The particular values of this mode frequency shows perfect agreement with the theoretical prediction. This means that the impurity mode is independent from the choice of initial conditions.

Apart from that, for small initial velocities of the waves in a kink-impurityantikink setup, we observe a quasi-long-lived solution with a small amplitude that is called an oscillon. The breathers and oscillons are similar objects because they both are quasi-long-lived oscillating solutions. However, the first one is characterized by a high amplitude, and its exact structure is still unknown, while the second one has a small amplitude, and its structure is well-known and has a form of a Gaussian. Originally, they were introduced in $(1+3)$ dimensions [190] in the $\varphi^{4}$ model, later they were implemented for lower dimensions [191] and some other models, e.g., the signum-Gordon model [192]. Usually, oscillons are used as ansatz [70, 71], however, the appearance of oscillons was observed beforehand for the sinh-deformed $(1+1)$-dimensional $\varphi^{4}$ model [74] after the procedure of a model deformation [72, 73]. To the best of our knowledge, we describe here the first time of the natural birth of oscillons in the classical $(1+1)$-dimensional $\varphi^{4}$ model. Apart from mathematical interest, these objects can be useful for developing models of cosmic inflationary [193].

For future research, it might be interesting to study systems with two pointlike impurities for single kink and the kink-antikink pair placed in between two 
6 Kinks and realistic impurity models in the $\varphi^{4}$ theory

impurities, specifically the influence of these initial conditions on the described above processes. A similar protocol was realized for multi-solitons in the sineGordon model [29], and the results look promising for describing magnetization of localized waves in a ferromagnet. 


\section{Appendix}

\section{A Numerical method}

A more general overview about numerically solving the equation of motion was already given in Chapter 5 (see Appendix 5.A). Here, we specify the equation of motion and the energy for the particular case that is considered in this Chapter.

To integrate the equation of motion in Eq. (6.2), we use finite differences on a discretized lattice in both space and time:

$$
\begin{aligned}
\varphi_{j}^{n+1}= & 2 \varphi_{j}^{n}-\varphi_{j}^{n-1}+\frac{\Delta t^{2}}{\Delta x^{2}}\left(\varphi_{j+1}^{n}-2 \varphi_{j}^{n}+\varphi_{j-1}^{n}\right) \\
& -\left.\Delta t^{2}\left(\varphi^{3}-\varphi\right)\right|_{\varphi=\varphi_{j}^{n}}\left(1-\epsilon \gamma\left(x_{j}-x_{0}\right)\right) .
\end{aligned}
$$

We compute the change in the total energy, which should be close to zero:

$$
\begin{aligned}
\Delta E_{\text {tot }}\left(n^{\prime}\right) & =E\left(n=n^{\prime}\right)-E(n=0)-\sum_{n=0}^{n^{\prime}}\left[\frac{\partial \varphi}{\partial t} \frac{\partial \varphi}{\partial x}\right]_{j=-M / 2+N}^{M / 2-N} \\
\text { with } E(n) & =\sum_{j=-M / 2+N}^{M / 2-N}\left(\frac{1}{2}\left(\frac{\partial \varphi}{\partial t}\right)^{2}+\frac{1}{2}\left(\frac{\partial \varphi}{\partial x}\right)^{2}+\frac{1}{4}\left(1-\varphi^{2}\right)^{2}\left(1-\epsilon \gamma\left(x-x_{0}\right)\right)\right) .
\end{aligned}
$$

For simulations it turns out that $\Delta t=\tau=0.01$ and $\Delta x=h=0.02$ are reasonable values for choices $t_{\max }=350$ and $x_{\max }=730$. The maximal detected fluctuations in the energy during the time evolution of an initial field configuration were less than $0.74 \%$ (for the kink) and $1.06 \%$ (for the kink-antikink pair), indicating the stability and accuracy of the numerical routine.

\section{B Frequency and amplitude}

To extract the frequency and amplitude of the impurity mode oscillations from the numerically obtained field profile, we can either use a discrete Fourier transform of $\varphi\left(x_{0}, t\right)$, or directly average the distances between its observed minima and maxima. In either approach, we only consider the field values for $t>170$ to ensure that the kink has entirely passed by the impurity center. The accuracy of both methods is limited by the discrete sampling of the continuous field $\varphi\left(x_{0}, t\right)$, and the values obtained for $A$ and $\tilde{\Omega}$ with the two methods do not differ significantly. 


\section{C Collective coordinate approximation}

Colliding kink and antikink in the $\varphi^{4}$ theory behave in many aspects like colliding hard-core particles with a short-range attractive interaction. If the kinetic energy of these particles is sufficiently large, the particles scatter without being affected by their short-ranged attraction. If the kinetic energy is low enough, however, the attraction will dominate, and a bound state will be formed. This qualitative observation can be made quantitative by constructing a low-energy effective theory for the $\varphi^{4}$ model, in which only the dynamics of kinks is taken into account, and all other variations in the field are neglected. Such an effective theory is known as the Collective Coordinate Approximation, or CCA [64]. Originally [194], the "collective coordinates" which correspond to the centers of the kinks and the amplitudes of the small oscillations around them were used to write the effective Lagrangian to discuss the "emission" of small oscillations ("bosons") from the kinks, however, not to qualitatively analyze kink-antikink interactions. A historical account can be found in $[156]$.

For the sake of concreteness, consider the case of colliding kink and antikink. In its simplest form, the CCA consists of constructing a theory in which the only degrees of freedom are the positions of the waves [64]. Going to a frame of reference in which the configuration is symmetric around $x=0$, there is only a single degree of freedom $a(t)$ describing the positions of the two waves. At any point in time, the field configuration (is presented in Fig. 6.7) associated with a given value of the collective coordinate $a$ is then approximated by

$$
\varphi_{K \bar{K}}(x)=\tanh \left(\frac{x+a}{\sqrt{2}}\right)-\tanh \left(\frac{x-a}{\sqrt{2}}\right)-1 .
$$

The effective Lagrangian for the collective coordinate, $L_{c c a}(a, \dot{a})$, is obtained by substituting the ansatz of Eq. (6.14) into the Lagrangian density for the full $\varphi^{4}$ theory, Eq. (4.1) with Eq. (4.13), and integrating it over space. Taking into account the time dependence of $a(t)$ in the calculation of $\partial \varphi / \partial t$, this leads to a Lagrangian of the form:

$$
L_{c c a}(a, \dot{a})=\frac{1}{2} m(a) \dot{a}^{2}-V(a)
$$

The position-dependent mass and potential energy in this expression can be written 


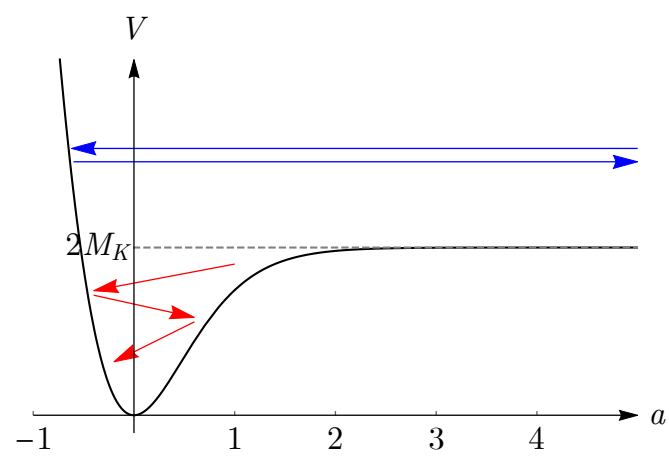

Figure 6.15: The plot of $V(a)$ from the Eq. (6.16). The gray dashed line corresponds to $2 M_{K}$. The blue arrows and the red arrows answer correspond to inelastic reflection (infinite motion) and formation of a quasi-breather (finite motion) correspondingly.

in terms of an auxiliary function as:

$$
\begin{aligned}
m(a) & =I_{+}(a) \\
V(a) & =\frac{1}{2} I_{-}(a)+\frac{1}{4} \int_{-\infty}^{+\infty}\left(1-\varphi_{K \bar{K}}^{2}\right)^{2} d x \\
\text { with } \quad I_{ \pm}(a) & =2 M_{K} \pm \int_{-\infty}^{+\infty} \frac{d x}{\cosh ^{2}((x+a) / \sqrt{2}) \cosh ^{2}((x-a) / \sqrt{2})} .
\end{aligned}
$$

At large separation, the integral in the auxiliary functions vanishes, and we find that the mass parameter $m(a)$ and the effective potential $V(a)$ approach $2 M_{K}=4 \sqrt{2} / 3$, the mass of two isolated static kinks (see Section 4.5 in Chapter 4). For general values of $a$, the integrals can be evaluated numerically, and yield the functional form for the effective potential plotted in Fig. 6.15.

The effective Lagrangian of Eq. (6.15) has reduced the initial relativistic field theory to a nonrelativistic description of just a single point particle in an external potential. This can be easily solved by considering the Euler-Lagrange equation for the collective coordinate:

$$
\begin{aligned}
\frac{d}{d t} \frac{\partial L_{c c a}}{\partial a}-\frac{\partial L_{c c a}}{\partial a} & =0 \\
\Rightarrow \quad m \ddot{a}+\frac{1}{2} \frac{d m}{d a} \dot{a}^{2}+\frac{d V}{d a} & =0 .
\end{aligned}
$$


The velocity-dependent term in the final line may be interpreted as a frictional force acting on the effective particle. This arises due the change of the effective mass $m(a)$ with position, which is significant only when the kink and the antikink overlap. It is thus a direct manifestation of the two-kink configuration not being a stable solution to the equation of motion for the full field theory.

Based on the shape of the effective potential $V(a)$ shown in Fig. 6.15, we can distinguish two qualitatively different types of dynamics. If the initial energy of the particle described by $a(t)$ is sufficiently high, it can cross the potential well around the origin $a=0$, climb up the potential barrier, which increases linearly with $|a|$ at negative $a$, then turn around and return through the well before escaping to infinity. This describes the bouncing of the kink and the antikink. If the initial energy is low enough, however, the particle may loose sufficient energy during its crossing of the potential well to become stuck. It will then oscillate back and forth across $a=0$ with slowly decreasing amplitude. This describes the formation of a quasi-long-lived bion.

In many cases, numerically solving Eq. (6.19) for the effective model yields good agreement with the much more involved numerical solutions for the dynamics of the full field-theoretical model [59]. The ansatz of Eq. (6.14) does neglect the Lorentz contraction of moving waves, so that the CCA fails to reproduce relativistic effects that may appear at high velocities. Another limitation of the CCA is that it cannot describe the escape windows, because it does not include degrees of freedom to describe the internal excitation modes of the kink and the antikink. Extensions of the CCA that include resonance dynamics are possible [67].
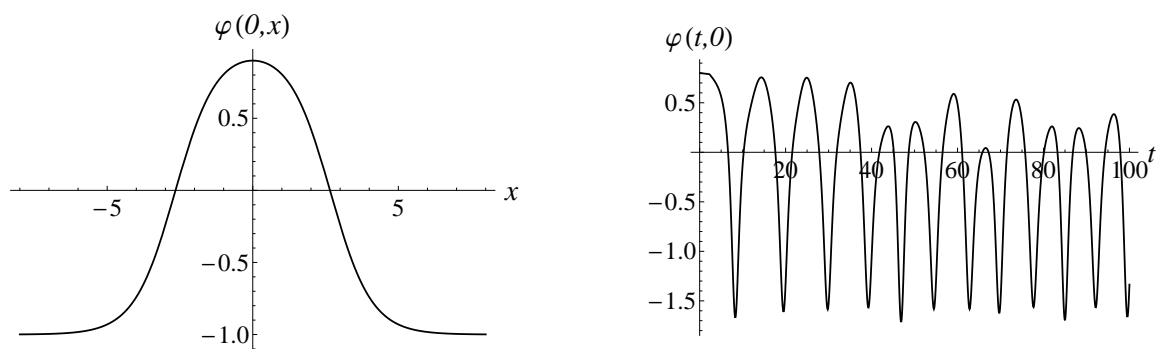

Figure 6.16: The profile of $\varphi(t, x)$ at $t=0$. Parameters: $\varphi_{0}=0.8$ and $\delta \varphi=$ -0.001 (left panel). The profile of $\varphi(t, 0)$. Parameters: $\varphi_{0}=0.8$ and $\delta \varphi=-0.001$ (right panel). Reprinted from [195]. 


\section{D Kink-elliptic sine-antikink ansatz}

The quasi-long-lived bion that we described as a damped oscillator, can also be thought of as a combination of parts of three static solutions to the Eq. (4.14). Just considering the shape of the field configuration $\varphi(t, x)$ for a bion at some time $t$, you may notice that it looks like half a kink and half an antikink, glued together by half a period of the elliptic sine, as shown in Fig. 6.16. In fact, we can make this more precise by constructing a field configuration starting from the static solution in Eq. (4.17). Taking a parameter $\lambda$ to be the period of the elliptic sine, we keep only half a period, within the range $-\lambda / 4<x<\lambda / 4$. We then attach half a kink to the left, for $-\infty<x<\lambda / 4$, and half an antikink to the right, for $\lambda / 4<x<\infty$. The positions $a=-\lambda / 4$ of the kink and $a=\lambda / 4$ of the antikink are chosen such that there are no discontinuities in the field. The value of $\varphi_{0}$, and hence $\lambda$, has to be chosen such that the three static solutions glue together smoothly at the connection points $x= \pm \lambda / 4$. We can then consider this configuration of glued static solutions as an ansatz for the bion field, and calculate its time evolution.

Notice that in the numerical calculation of the bion dynamics starting from this ansatz $\varphi(t, x)$, the field at time $t+\delta t$ also needs to be defined. For this, we can simply choose to decrease the value of $\varphi_{0}$ by a small amount $\delta \varphi_{0}$. The result of the ensuing time evolution is shown in Fig. 6.16 (right panel), and closely resembles bion that found in the kink collisions.

The strategy of gluing together static solutions to find an appropriate ansatz or initial state may be applied more generally. For example, dynamical kink-kink pairs and triton solutions (kink-antikink-kink) were described this way [195]. The latter may be used as an ansatz for obtaining the quasi-long-lived solution called a wobbling kink $[178,179]$. 



\section{Bibliography}

[1] B. A. Bernevig, T. L. Hughes, and S.-C. Zhang, Science 314, 1757 (2006).

[2] M. Koenig, S. Wiedmann, C. Bruene, A. Roth, H. Buhmann, L. W. Molenkamp, X.-L. Qi, and S.-C. Zhang, Science 318, 766 (2007).

[3] C. L. Kane and E. J. Mele, Phys. Rev. Lett. 95, 226801 (2005).

[4] C. L. Kane and E. J. Mele, Phys. Rev. Lett. 95, 146802 (2005).

[5] X.-L. Qi and S.-C. Zhang, Phys. Today 63, 33 (2010).

[6] M. Z. Hasan and C. L. Kane, Rev. Mod. Phys. 82, 3045 (2010).

[7] X.-L. Qi and S.-C. Zhang, Rev. Mod. Phys. 83, 1057 (2011).

[8] R.-J. Slager, A. Mesaros, V. Juričić, and J. Zaanen, Nature Physics 9, 98 (2013).

[9] K. von Klitzing, G. Dorda, and M. Pepper, Phys. Rev. Lett. 45, 494 (1980).

[10] Q. Niu, Phys. Rev. B 34, 5093 (1986).

[11] L. Wang, M. Troyer, and X. Dai, Phys. Rev. Lett. 111, 026802 (2013).

[12] D. J. Thouless, Phys. Rev. B 27, 6083 (1983).

[13] F. Flicker and J. van Wezel, EPL (Europhysics Letters) 111, 37008 (2015).

[14] R. B. Laughlin, Phys. Rev. B 23, 5632 (1981).

[15] V. T. Dolgopolov, A. A. Shashkin, S. I. Zhitenev, N. B. Dorozhkin, and K. von Klitzing, Phys. Rev. B 46, 12560 (1992).

[16] A. B. Vorob'ev, V. Y. Prinz, Y. S. Yukecheva, and A. I. Toropov, Physica E 23, 171 (2004). 
Bibliography

[17] P. G. Harper, Proc. Phys. Soc. Lond. A 68, 874 (1955).

[18] J. Bellissard and B. Simon, J. Funct. Anal. 48, 408 (1982).

[19] D. R. Hofstadter, Phys. Rev. B 14, 2239 (1976).

[20] B. D. Josephson, Phys. Lett. 1, 251 (1962).

[21] P. W. Anderson and J. M. Rowell, Phys. Rev. Lett. 10, 230 (1963).

[22] J. Pfeiffer, M. Schuster, A. A. Abdumalikov, Jr., and A. V. Ustinov, Phys. Rev. Lett. 96, 034103 (1996).

[23] Z. Hermon, E. Ben-Jacob, and G. S. n, Phys. Rev. B 54, 1234 (1996).

[24] G. Costabile, S. Pagano, and S. Pedersen, Nonlinear Superconductive Electronics and Josephson Devices (Plenum Press, New York, 1991) [p. 315].

[25] V. N. Ginzburg and L. D. Landau, Zh. Exsp. Teor. Fiz. 20, 1064 (1950), [English version in Collected papers (Oxford: Pergamon Press), p. 546-568 (1965)].

[26] P. W. Higgs, Phys. Rev. 145, 1156 (1983).

[27] Z. Fei, Y. S. Kivshar, and L. Vazquez, Phys. Rev. A 45, 6019 (1992).

[28] R. H. Goodman and R. Haberman, Physica D 195, 303 (2004).

[29] E. G. Ekomasov, A. M. Gumerov, R. V. Kudryavtsev, S. V. Dmitriev, and V. N. Nazarov, Braz. J. Phys. 48, 576 (2018).

[30] B. A. Malomed, Physica D 15, 385 (1985).

[31] K. Javidan, J. Phys. A: Math. Gen. 39, 10565 (2006).

[32] B. A. Malomed, Journal of Physics A: Mathematical and General 25, 755 (1992).

[33] Z. Fei, Y. S. Kivshar, and L. Vazquez, Phys. Rev. A 46, 5214 (1992).

[34] Y. S. Kivshar, Z. Fei, and L. Vazquez, Phys. Rev. Lett. 67, 1177 (1991).

[35] Z. Fei, V. V. Konotop, M. Peyrard, and L. Vazquez, Phys. Rev. E 48, 548 (1993).

[36] K. Javidan, Phys. Rev. E 78, 046607 (2008).

[37] E. Hakimi and K. Javidan, Phys. Rev. E 80, 016606 (2009). 
[38] A. Ghahraman and K. Javidan, Brazilian J. Phys. 41, 171 (2011).

[39] M. B. Fogel, S. E. Trullinger, A. R. Bishop, and J. A. Krumhansl, Phys. Rev. B 15, 1578 (1977).

[40] S. Hu, M. Lundgren, and A. J. Niemi, Phys. Rev. E 83, 061908 (2011).

[41] S. Hu, A. Krokhotin, A. J. Niemi, and X. Peng, Phys. Rev. E 83, 041907 (2011), [arXiv:1011.3181 [q-bio.BM]].

[42] A. Molochkov, A. Begun, and A. Niemi, EPJ Web of Conferences 137, 04004 (2017).

[43] D. Whitford, Proteins: Structure and Function (John Wiley Sons, England, 2005).

[44] C. M. Dobson, Nature 426, 884 (2003).

[45] K. A. Dill and J. L. MacCallum, Science 338, 1042 (2012).

[46] I. Kuznetsova, V. Forge, and K. Turoverov, Tsitologiia 47, 943 (2005), [In Russian].

[47] F. Chiti and C. M. Dobson, Annual Review of Biochemistry 86, 27 (2017).

[48] D. Hanahan and R. A. Weinberg, Cell 100, 57 (2000).

[49] D. Hanahan and R. A. Weinberg, Cell 144, 646 (2011).

[50] J. Wu, T. Liu, Z. Rios, Q. Mei, X. Lin, and S. Cao, Trends in Pharmacological Sciences 38, 226 (2017).

[51] F. M. LaFerla, K. N. Green, and S. Oddo, Nat. Rev. Neurosci. 8, 499 (2007).

[52] C. Soto, L. Estrada, and J. Castilla, Trends in Biochemical Sciences 31, 150 (2006).

[53] S. Makin, Nature 559, S4 (2018).

[54] P. D. Gorevic, UpToDate 10 (2013), [Retrieved February 7, 2019 from https://www.uptodate.com/contents/overview-of-amyloidosis].

[55] A. H. S. Martinelli, F. C. Lopes, E. B. O. John, C. R. Carlini, and R. LigabueBraun, Int. J. Mol. Sci. 20, 1322 (2019).

[56] S. Sinha, D. H. J. Lopes, Z. Du, E. S. Pang, A. Shanmugam, A. Lomakin, P. Talbiersky, A. Tennstaedt, K. McDaniel, R. Bakshi, P.-Y. Kuo, M. Ehrmann, G. B. Benedek, J. A. Loo, F.-G. Klärner, T. Schrader, C. Wang, and G. Bitan, J. Am. Chem. Soc. 133, 16958 (2011). 
Bibliography

[57] B. T. V. L. T. Vassilev and, B. Graves, D. Carvajal, F. Podlaski, Z. Filipovic, N. Kong, U. Kammlott, C. Lukacs, C. Klein, N. Fotouhi, and E. A. Liu, Science 303, 844 (2004).

[58] C. Tovar, J. Rosinski, Z. Filipovic, B. Higgins, K. Kolinsky, H. Hilton, X. Zhao, B. T. Vu, W. Qing, K. Packman, O. Myklebost, D. C. Heimbrook, and L. T. Vassilev, PNAS 103, 1888 (2006).

[59] T. I. Belova and A. E. Kudryavtsev, Usp. Fiz. Nauk 167, 377 (1997), [Sov. Phys. Usp. 40, 359 (1997)].

[60] M. J. Ablowitz, M. D. Kruskal, and J. F. Ladik, SIAM J. Appl. Math. 36, 428 (1979).

[61] D. K. Campbell, J. S. Schonfeld, and C. A. Wingate, Phys. D 9, 1 (1983).

[62] P. Anninos, S. Oliveira, and R. A. Matzner, Phys. Rev. D 44, 1147 (1991).

[63] R. Goodman and R. Haberman, SIAM J. Appl. Math. Dyn. Syst. 4, 1195 (2005).

[64] A. E. Kudryavtsev, Pis'ma Zh. Eksp. Teor. Fiz. 22, 178 (1975), [JETP Lett. 22, $82(1975)]$.

[65] T. Sugiyama, Prog. Theor. Phys. 61, 1550 (1979).

[66] R. H. Goodman, Chaos: An Interdisciplinary Journal of Nonlinear Science 18, 023113 (2008).

[67] I. Takyi and H. Weigel, Phys. Rev. D 94, 085008 (2016).

[68] H. Weigel, J. Phys.: Conf. Ser. 482, 012045 (2014).

[69] R. H. Goodman, A. Rahman, M. J. Bellanich, and C. N. Morrison, Chaos 25, 043109 (2015).

[70] C. S. Carvalho and L. Perivolaropoulos, Phys. Rev. D 79, 065032 (2009).

[71] T. Romanczukiewicz and Y. Shnir, Phys. Rev. Lett. 105, 081601 (2010).

[72] D. Bazeia, L. Losano, and J. M. C. Malbouisson, Phys. Rev. D 66, 101701(R) (2002).

[73] D. Bazeia, M. A. G. León, L. Losano, and J. M. Guilarte, Phys. Rev. D 73, 105008 (2006).

[74] D. Bazeia, E. Belendryasova, and V. A. Gani, Eur. Phys. J. C 78, 340 (2018). 
[75] D. J. Thouless, M. Kohmoto, M. P. Nightingale, and M. den Nijs, Phys. Rev. Lett. 49, 405 (1982).

[76] J. E. Avron and R. Seiler, Phys. Rev. Lett. 54, 259 (1985).

[77] A. Tzalenchuk, S. Lara-Avila, A. Kalaboukhov, S. Paolillo, M. Syvajarvi, R. Yakimova, O. Kazakova, T. J. B. M. Janssen, V. Fal'ko, and S. Kubatkin, Nature Nanotech. 5, 186 (2010).

[78] J. Kruthoff, J. de Boer, J. van Wezel, C. L. Kane, and R.-J. Slager, Phys. Rev. X 7, 041069 (2017).

[79] B. I. Halperin, Phys. Rev. B 25, 2185 (1982).

[80] Y. Hatsugai, Phys. Rev. Lett. 71, 3697 (1993).

[81] V. Gritsev and A. Polkovnikov, PNAS 109, 6457 (2012).

[82] M. A. Lizunova, F. Schreck, C. Morais Smith, and J. van Wezel, Phys. Rev. B 99, 115114 (2019).

[83] D. Xiao, M.-C. Chang, and Q. Niu, Rev. Mod. Phys. 82, 1959 (2010).

[84] S. Nakajima, T. Tomita, S. Taie, T. Ichinose, H. Ozawa, L. Wang, M. Troyer, and Y. Takahashi, Nat. Phys. 12, 296 (2016).

[85] M. Lohse, C. Schweizer, O. Zilberberg, M. Aidelsburger, and I. Bloch, Nat. Phys. 12, 350 (2016).

[86] W. Ma, L. Zhou, Q. Zhang, M. Li, C. Cheng, J. Geng, X. Rong, F. Shi, J. Gong, and J. Du, Phys. Rev. Lett. 120, 120501 (2018).

[87] B. L. Altshuler and L. I. Glazman, Science 283, 1864 (1999).

[88] M. Switkes, C. M. Marcus, K. Campman, and A. C. Gossard, Science 283, 1905 (1999).

[89] M. Moskalets and M. Buttiker, Phys. Rev. B 66, 205320 (2002).

[90] I. Maruyama and Y. Hatsugai, J. Phys.: Conf. Ser. 150, 022055 (2009).

[91] D. Tsui, H. H. Stormer, and A. Gossard, Phys. Rev. Lett. 48, 1559 (1982).

[92] R. B. Laughlin, Phys. Rev. Lett. 50, 1395 (1983).

[93] F. D. M. Haldane, Phys. Rev. Lett. 61, 2015 (1988).

[94] A. C. Potter and P. A. Lee, Phys. Rev. B 85, 094516 (2012). 
Bibliography

[95] C. Chiu, J. Teo, A. Schnyder, and S. Ryu, Rev. Mod. Phys. 88, 035005 (2016).

[96] A. Altland and M. R. Zirnbauer, Phys. Rev. B 55, 1142 (1997).

[97] S. Ryu, A. Schnyder, A. Furusaki, and A. Ludwig, New J. Phys. 12, 065010 (2010).

[98] J. Kruthoff, J. de Boer, and J. van Wezel, Phys. Rev. B 100, 075116 (2019).

[99] H. C. Po, A. Vishwanath, and H. Watanabe, Nature Commun. 8, 50 (2017).

[100] H. Watanabe, H. C. Po, and A. Vishwanath, Science Adv. 4, eaat8685 (2018).

[101] B. Bradlyn, L. Elcoro, J. Cano, M. G. Vergniory, Z. Wang, C. Felser, M. I. Aroyo, and B. A. Bernevig, Nature 547, 298 (2017).

[102] M. A. Goerbig, arXiv:0909.1998 [cond-mat.mes-hall] [Lecture notes for the Singapore session "Ultracold Gases and Quantum Information" of Les Houches Summer School].

[103] G. Grüner, Density Waves in Solids (Perseus Books Group, 2000).

[104] R. Peierls, Ann. d. Phys. Leipzig 4, 121 (1930).

[105] M.-C. Chang and Q. Niu, Phys. Rev. Lett. 75, 1348 (1995).

[106] G. Grüner, Rev. Mod. Phys. 60, 1129 (1988).

[107] L.-C. Ha, L. W. Clark, C. V. Parker, B. M. Anderson, and C. Chin, Phys. Rev. Lett. 114, 055301 (2015).

[108] B. Huckestein, Rev. Mod. Phys. 67, 357 (1995).

[109] J. T. Chalker, J. Phys. C 20, L493 (1987).

[110] O. Lychkovskiy, O. Gamayun, and V. Cheianov, Phys. Rev. Lett. 119, 200401 (2017).

[111] O. Lychkovskiy, O. Gamayun, and V. Cheianov, AIP Conf. Proc. 1936, 020024 (2018).

[112] A. Polkovnikov and V. Gritsev, Nature Phys. 4, 477 (2008).

[113] P. C. Snijders, S. Rogge, and H. H. Weitering, Phys. Rev. Lett. 96, 076801 (2006).

[114] C. Brun, Z.-Z. Wang, P. Monceau, and S. Brazovskii, Phys. Rev. Lett. 104, $256403(2010)$. 
[115] A. Soumyanarayanan, M. M. Yee, Y. He, J. van Wezel, D. J. Rahn, K. Rossnagel, E. W. Hudson, M. R. Norman, and J. E. Hoffman, PNAS 110, 1623 (2013).

[116] M. M. Ugeda, A. J. Bradley, Y. Zhang, S. Onishi, Y. Chen, W. Ruan, C. Ojeda-Aristizabal, H. Ryu, M. T. Edmonds, H.-Z. Tsai, A. Riss, S.-K. Mo, D. Lee, A. Zettl, Z. Hussain, Z.-X. Shen, and M. F. Crommie, Nat. Phys. 12, 92 (2016).

[117] Y. E. Kraus, Y. Lahini, Z. Ringel, M. Verbin, and O. Zilberberg, Phys. Rev. Lett. 109, 106402 (2012).

[118] M. Lohse, C. Schweizer, H. M. Price, O. Zilberberg, and I. Bloch, Nature 553, 55 (2018).

[119] W. S. Bakr, J. I. Gillen, A. Peng, S. Fölling, and M. Greiner, Nature 426, $74(2009)$.

[120] M. A. Norcia, A. W. Young, and A. M. Kaufman, Phys. Rev. X 8, 041054 (2018).

[121] A. Scott, The Encyclopedia of Nonlinear Science (Routledge, New York, 2004) Chap. Solitons, a brief history, p. 852.

[122] N. J. Zabusky and M. D. Kruskal, Phys. Rev. Lett. 15, 240 (1965).

[123] N. S. Manton and P. M. Sutcliffe, Topological solitons (Cambridge University Press, Cambridge, 2004).

[124] J. K. Perring and T. H. R. Skyrme, Nuclear Phys. 31, 550 (1962).

[125] A. Vilenkin and E. P. S. Shellard, Cosmic stings and other topological defects (Cambridge University Press, Cambridge, 1994).

[126] A. Friedland, H. Murayama, and M. Perelstein, Phys. Rev. D 67, 043519 (2003).

[127] Z. Chen, M. Segev, and D. N. Christodoulides, Rep. Prog. Phys. 75, 086401 (2012).

[128] G. P. Agrawall, Nonlinear Fiber Optics (CA: Academic Press, San Diego, 1995).

[129] A. R. Bishop and T. Schneider, in Proceedings of the Symposium on Nonlinear (Soliton) Structure and Dynamics in Condensed Matter (Oxford, 1978). 
Bibliography

[130] K. E. Strecker, G. B. Patridge, A. G. Truscott, and R. G. Hulet, New J. Phys. 5, 73.1 (2003).

[131] Y. N. Gornostyrev, M. I. Katsnelson, A. V. Kravtsov, and A. V. Trefilov, Phys. Rev. E. 66, 027201 (2002).

[132] L. Brizhik, JAP 6, 1191 (2014).

[133] J. Weiss, J. Math. Phys. 25, 2226 (1984).

[134] R. Rajaraman, Solitons and Instantons: An Introduction to Solitons and Instantons in Quantum Field Theory (North-Holland Publishing Company, 1982).

[135] G. Bykov, J. Seismol. 18, 497 (2014).

[136] M. Lowe and J. P. Gollub, Phys. Rev. A 31, 3893 (1985).

[137] J. J. Mazo and A. V. Ustinov, Nonlinear Systems and Complexity 10, 155 (2014).

[138] Y. S. Gal'Pern and A. T. Fillipov, Zh. Eksp. Teor. Fiz. 86, 1527 (1984).

[139] K. Sasaki and K. Maki, Phys. Rev. B 35, 257 (1987).

[140] P. Dorey, K. Mersh, T. Romanczukiewicz, and Y. Shnir, Phys. Rev. Lett. 107, 091602 (2011).

[141] V. A. Gani, A. E. Kudryavtsev, and M. A. Lizunova, Phys. Rev. D 89, 125009 (2014).

[142] V. A. Gani, V. Lensky, and M. A. Lizunova, JHEP 8, 147 (2015).

[143] Y. Tan and J. Yang, Phys. Rev. E 64, 56616 (2001).

[144] A. Halavanau, T. Romanczukiewicz, and Y. Shnir, Phys. Rev. D 86, 085027 (2012).

[145] A. Alonso-Izquierdo, Phys. Rev. D 97, 045016 (2018).

[146] M. Remoissenet and M. Peyrard, Phys. Rev. B 29, 3153 (1984).

[147] D. Stojkovic, Phys. Rev. D 63, 025010 (2000).

[148] D. Bazeia, L. Losano, and J. M. C. Malbouisson, Phys. Rev. D 66, 101701(R) (2002).

[149] D. Bazeia, in Proceedings of XIII J.A. Swieca Summer School on Particles and Fields (SP, 2005). 
[150] R. Rajaraman, Phys. Rep. C 21, 227 (1975).

[151] A. B. Adib and C. A. S. Almeida, Phys. Rev. E 64, 037701 (2001).

[152] A. J. Beekman, L. Rademaker, and J. van Wezel, SciPost Phys. Lect. Notes 11 (2019).

[153] E. B. Bogomolny, Yad. Fiz. 24, 861 (1976), [Sov. J. Nucl. Phys. 24, 449 (1976)].

[154] M. K. Prasad and C. M. Sommerfield, Phys. Rev. Lett. 35, 760 (1975).

[155] J. K. Perring and T. H. R. Skyrme, Nucl. Phys. 31, 550 (1962).

[156] D. K. Campbell, A Dynamical Perspective on the $\phi^{4}$ Model: Past, Present, and Future (Springer, 2019) [p. 1-22, Historical Overview of the $\phi^{4}$ model].

[157] T. H. Kyaw, Towards a Scalable Quantum Computing Platform in the Ultrastrong Coupling Regime (Springer International Publishing, 2019).

[158] M. Remoissenet, Waves Called Solitons (Springer, Berlin, 1999) [p. 190-203, Chapter 7. Fluxons in Josephson Transmission Lines].

[159] A. V. Ustinov, Physica D 123, 315 (1998).

[160] A. Davidson, B. Dueholm, B. Kryger, and N. F. Pedersen, Phys. Rev. Lett. 55, 1985 (2059).

[161] A. V. Ustinov, T. Doderer, R. P. Huebener, N. F. Pedersen, B. Mayer, and V. A. Oboznov, Phys. Rev. Lett. 69, 1815 (1992).

[162] I. V. Vemik, N. Lazarides, M. P. Sørensen, A. V. Ustinov, N. F. Pedersen, and V. Oboznov, J. Appl. Phys. 79, 7854 (1996).

[163] A. Laub, T. Doderer, S. G. Lachenmann, R. P. Huebner, and V. A. Oboznov, Phys. Rev. Lett. 75, 1372 (1995).

[164] S. G. Lachenmann, T. Doderer, R. P. Huebener, D. Quenter, J. Niemeyer, and R. Pöpel, Phys. Rev. B 48, 3295 (1993).

[165] S. G. Lachenmann, G. FilatreUa, A. V. Ustinov, T. Doderer, N. Kirchmann, D. Quenter, R. Huebner, J. Niemeyer, and R. Pöpel, J. Appl. Phys. 77, 2598 (1995).

[166] D. Quenter, A. V. Ustinov, S. G. Lachenmann, T. Doderer, R. P. Huebener, E. Miiller, J. Niemeyer, R. Pöpel, and T. Weimann, Phys. Rev. B 51, 6542 (1995). 
Bibliography

[167] S. Keil, T. Doderer, I. V. Vemik, A. Laub, H. Pressler, R. P. Huebener, N. Thyssen, A. V. Usfinov, and H. Kohlstedt, Phys. Rev. B 54, 14948 (1996).

[168] G. S. Mkrtychyan and V. V. Shmidt, Solid State Commun. 30, 783 (1979).

[169] J. F. Currie, S. E. Trullinger, A. R. Bishop, and J. A. Krumhansl, Phys. Rev. B 15, 5567 (1977).

[170] A. Kundu, Phys. Rev. Lett. 99, 154101 (2007).

[171] A. Kundu, J. Nonlinear. Math. Phys. 15, 237 (2008).

[172] Y. Yang, Z.-H. Tian, and J.-L. Jing, Chin. Phys. Lett. 37, 048501 (2020).

[173] J. Kendrew, Nature 181, 662 (1958).

[174] H. M. Berman, K. Henrick, H. Nakamura, and J. L. Markley, Nucl. Acids Res. 35, D301 (2007).

[175] P. L. Freddolino, C. B. Harrison, Y. Liu, and K. Schulten, Nature Phys. 6, 751 (2010).

[176] K. L. Larsen, S. Piana, R. Dror, and D. Shaw, Science 334, 517 (2011).

[177] L. D. Landau and E. M. Lifshitz, Quantum Mechanics Non-relativistic theory (Pergamon Press, London, 1965) [p. 72, Section 23, Problem 4].

[178] B. S. Getmanov, Pis'ma Zh. Eksp. Teor. Fiz. 24, 323 (1976), [JETP Lett. 24, 291 (1976)].

[179] O. F. Oxtoby and I. V. Barashenkov, Teor. Mat. Fiz. 159, 527 (2009), [Theoret. and Math. Phys. 159, 863 (2009)].

[180] M. Abramowitz and I. A. Stegun, Handbook of Mathematical Functions with Formulas, Graphs, and Mathematical Tables (Dover, New York, 1964).

[181] A. M. Kosevich, Y. S. Kivshar, and O. A. Chubykalo, Fiz. Nizk. Temp. 13, 800 (1987), [In Russian].

[182] V. M. Krasnov, V. A. Oboznov, and N. F. Pedersen, Phys. Rev. B 55, 14486 (1997).

[183] O. Gamayun and M. Semenyakin, Journal of Physics A: Mathematical and Theoretical 49, 335201 (2016).

[184] O. Gamayun, Y. V. Bezvershenko, and V. Cheianov, Phys. Rev. A 91, 031605 (2015). 
[185] Y. Miao, E. Ilievski, and O. Gamayun, Phys. Rev. A 99, 023605 (2019).

[186] O. Gamayun, Y. Miao, and E. Ilievski, Phys. Rev. B 99, 140301 (2019).

[187] A. Wallraff, Y. Koval, M. Levitchev, M. V. Fistul, and A. V. Ustinov, J. Low Temp. Phys. 118, 543 (2000).

[188] R. Courant, K. Friedrichs, and H. Lewy, Math. Ann. 100, 32 (1928).

[189] A. Askari, D. Saadatmand, and K. Javidan, Waves in Random and Complex Media (2018).

[190] E. J. Copeland, M. Gleiser, and H.-R. Mueller, Phys. Rev. D 52, 1920 (1995).

[191] A. B. Adib, M. Gleiser, and C. A. S. Almeida, Phys. Rev. D 66, 085011 (2002).

[192] F. M. Hahne, P. Klimas, J. S. Streibel, and W. J. Zakrzewski, JHEP 2020, 6 (2020).

[193] M. Gleiser, Int. J. Mod. Phys. D 16, 219 (2007).

[194] T. Sugiyama, Prog. Theor. Phys. 61, 550 (1979).

[195] A. E. Kudryavtsev and M. A. Lizunova, Phys. Rev. D 95, 056009 (2017), [arXiv:1610.09966 [hep-th]]. 



\section{Summary}

The purpose of this Thesis is to theoretically investigate the power of topology on various problems in condensed-matter physics, as well as their possible applications for biological systems.

In Chapter 3, we start the discussions with a well-known problem, the so-called Laughlin's gauge argument, i.e. quantized topological adiabatic transport in the integer quantum Hall cylinder. We describe singe-electron transport in the this system by using the mathematical equivalence between the two-dimensional integer quantum Hall effect and a one-dimensional charge-density wave, and reformulate the problem for the latter system. The obtained results yield adiabatic and nonadiabatic transport protocols for both setups. The theoretical predictions open prospects for possible experimental realizations in the context of ultracold atoms and photonic waveguide experiments.

In Chapter 5, we switch from topological insulators to the Josephson effect in a superconductor-insulator-superconductor setup. In experiment, an oscillatory current as a function of the phase difference between the two superconductors is observed, and the periodicity of this oscillation is set by the quantum of magnetic flux, so-called fluxon, that has a soliton-like character. It can be described with the modified sine-Gordon equation. In real life, one usually cannot avoid the presence of inhomogeneities in a system. In this chapter, we consider the behavior of topological solitary waves (solitons and kinks) in the sine-Gordon and the $\varphi^{4}$ classical field theories, in the presence of a step-like inhomogeneity. We observe that the wave evolution depends on the initial conditions, namely the initial velocity of a wave and the coupling constant of the potential. The soliton and kink movement can be either an elastic reflection or an inelastic propagation through the media with loss of kinetic energy and the excitation of small waves on top of the main wave, which can be interpreted as plasma waves in the Josephson effect. We observe that solitary wave behavior can be predicted by describing the latter as a relativistic particle. Most results are model-independent meaning that the integrable structure of the sine-Gordon model is irrelevant and robustness is rooted in the topology of these field configurations. Finally, we find a way to capture the moving wave in between 


\section{Summary}

two junctions. All mentioned results might be implemented in telecommunications or rapid single flux quantum logic through a fluxon diode.

In Chapter 6, we continue investigating single kink solutions of the $\varphi^{4}$ classical field theory, but now we verify its interaction with a single impurity described as a Dirac delta function, a Gaussian, or a Lorentzian. We find that kink behavior depends on the exact shape of the defect and on whether it is attractive or repulsive. We numerically prove the existence of an internal impurity mode, which already had been predicted theoretically beforehand. In the second part of Chapter 6 , we study a similar system, a single Gaussian impurity, which stays in between the kink and the antikink. We observe the excited internal impurity mode in this system as well. Apart from that, we see some other interesting phenomenon in the kink-impurity-antikink collisions. For some parameters of impurity strength and initial velocities of the kink and the antikink, a transformation of a kink-impurityantikink system into oscillon-impurity-oscillon occurs. The oscillon is another smallamplitude oscillating quasi-long-lived solution (a special form of a Gaussian) in the $\varphi^{4}$ model together with the high-amplitude quasi-breathers. We observe the bound state of two oscillons and the oscillons' escape windows by analogy with the quasibreather and resonances in the kink-antikink collisions. The kinks of the $\varphi^{4}$ model proved useful in the description of proteins. Despite the apparent simplicity of a problem of kink-impurity and kink-impurity-antikink interactions, these results might be useful in the future as a powerful tool for influence on a protein folding. 


\section{Samenvatting}

In de afgelopen decennia waren natuurkundigen creatief in het bedenken van wiskundige modellen voor werkelijk waargenomen verschijnselen, waarbij topologische eigenschappen een centrale rol spelen bij het begrijpen van het probleem. Het doel van dit Proefschrift is om de kracht van topologie op verschillende problemen in de fysica van de gecondenseerde materie theoretisch te onderzoeken, evenals hun mogelijke toepassingen voor biologische systemen.

In Hoofdstuk 3 beginnen we de discussies met een bekend probleem, het zogenaamde Laughlin ijkargument, met andere woorden gekwantificeerd topologisch adiabatisch transport in de kwantum-Hall-effect cilinder. We beschrijven het transport van elektronen in dit systeem door gebruik te maken van de wiskundige gelijkwaardigheid tussen het tweedimensionale integer quantum Hall-effect en een eendimensionale ladingsdichtheidsgolf, en herformuleren het probleem voor het laatstgenoemde systeem. De verkregen resultaten leveren adiabatische en niet-adabatische transport protocollen op voor beide opstellingen. De theoretische voorspellingen openen perspectieven voor mogelijke experimentele realisaties in de context van ultrakoude atomen en fotonische golfgeleider-experimenten.

In Hoofdstuk 5 schakelen we van topologische isolatoren naar een Josephsonjunctie in een supergeleider-isolator-supergeleider set-up. Bij een experiment wordt een oscillerende stroom als een functie van het faseverschil tussen de twee supergeleiders waargenomen. De periodiciteit van deze oscillatie wordt bepaald door het kwantum van de magnetische flux, het zogenaamde fluxon, dat een solitonachtig karakter heeft. Het kan worden beschreven met de gewijzigde sine-Gordon vergelijking. In de praktijk kan men de aanwezigheid van inhomogeniteiten in een systeem meestal niet vermijden. In dit hoofdstuk bestuderen we het gedrag van topologische solitaire golven (solitons en kinks) in de sine-Gordon en de $\varphi^{4}$ klassieke veldentheorieën in aanwezigheid van een stapachtige inhomogeniteit. We constateren dat de golfevolutie afhangt van de initiële omstandigheden, namelijk de beginsnelheid van een golf en van de variatie in de potentiaal. De soliton- en kinkbeweging kan een elastische reflectie zijn of een niet-elastische voortplanting met verlies van kinetische energie en de excitatie van kleine golven bovenop de hoofdgolf. Deze kleine gol- 


\section{Samenvatting}

ven kunnen worden geïnterpreteerd als plasmagolven in de Josephson-junctie. We stellen vast dat solitair golfgedrag kan worden voorspeld door de soliton te beschrijven als een relativistisch deeltje. De meeste resultaten zijn modelonafhankelijk, wat betekent dat de integreerbare structuur van het sine-Gordon-model niet relevant is en robuustheid is geworteld in de topologie van deze veldconfiguraties. Ten slotte vinden we een manier om de bewegende golf tussen twee knooppunten vast te leggen. Alle genoemde resultaten kunnen mogelijk worden geïmplementeerd in telecommunicatie of snelle kwantumlogica met een enkele flux via een fluxondiode.

In Hoofdstuk 6 gaan we door met het onderzoeken van enkele kinkoplossingen van de $\varphi^{4}$ klassieke veldtheorie, maar nu verifiëren we de interactie ervan met een enkele onzuiverheid die wordt beschreven als een Dirac-deltafunctie, Gaussische of Lorentziaanse functie. We vinden dat kinkgedrag afhangt van de exacte vorm van het defect en of dit aantrekt of afstoot. We bewijzen numeriek het bestaan van een interne excitatie van defecten, die vooraf al theoretisch was voorspeld. In het tweede deel van Hoofdstuk 6 bestuderen we een soortgelijk systeem, een enkele Gaussische onzuiverheid, tussen een kink en antikink. We observeren ook het aanslaan van de interne excitatie van de onzuiverheid in dit systeem. Afgezien daarvan zien we een ander interessant fenomeen in de botsingen tussen kink, onzuiverheid en antikink. Voor sommige parameters van onzuiverheidssterkte en beginsnelheden van de kink en de antikink, vindt een transformatie van een kink-onzuiverheid-antikink systeem naar oscillon-onzuiverheid-oscillon plaats. Het oscillon is een andere oscillerende quasi-langlevende oplossing met een kleine amplitude (een speciale vorm van een Gaussische golf) in het $\varphi^{4}$ model, samen met de gebonden kink-antikink toestanden met hoge amplitude. We observeren de gebonden toestand van twee oscillons en de ontsnappingsvensters van de oscillons naar analogie met de quasi-breather en resonanties in de kink-antikink-botsingen. De kinks van het $\varphi^{4}$-model bleken nuttig bij de beschrijving van proteïnen. Ondanks de schijnbare eenvoud van een probleem van kink-onzuiverheid en kink-onzuiverheid-antikink interacties, kunnen deze resultaten in de toekomst nuttig zijn als een krachtig hulpmiddel voor het beïnvloeden van het vouwen van proteïnen.

Ten slotte willen we erop wijzen dat dit onderzoek is begonnen in het najaar van 2016 in een andere wereld dan het in het voorjaar van 2020 is beeïndigd onder veranderde levensomstandigheden tijdens de coronacrisis of COVID-19-pandemie. Eén van de belangrijkste ideeën van dit proefschrift is te laten zien hoe de oplossing van bepaalde problemen kan helpen op de meest onverwachte gebieden van niet alleen natuurkunde, maar ook van de wetenschap als geheel. Dit is precies wat de maatschappij tegenwoordig nodig heeft: snelle, gracieuze oplossingen. Topologie, zoals we in dit proefschrift willen aantonen, zou een van die krachtige instrumenten kunnen zijn om dit te bereiken. 


\section{Curriculum Vitae}

Mariia Andreyevna Lizunova (professional name Mariya Lizunova) was born on the 19th of July, 1993 in Elektrostal (Moscow Oblast), Russia, but very soon her family moved to Moscow, where in 2010 she obtained a cum laude diploma at the state school 1324, specializing in English.

In 2010, she became a student in the National Research Nuclear University MEPhI (NRNU MEPhI) on the Faculty of Experimental and Theoretical Physics. In parallel, she studied advanced theoretical mechanics and quantum mechanics in the Lebedev Physical Institute RAS. In the end of the second year, she entered the Department for Theoretical Nuclear Physics. She successfully finished her bachelor (2014) and master cum laude (2016) thesis under the supervision of Dr. Alexander E. Kudryavtsev from the Institute for Theoretical and Experimental Physics (NRC Kurchatov Institute ITEP). In 2015, Mariia earned the status of the Best student of NRNU MEPhI. In the same year she won a scholarship of the Dmitry Zimin Dynasty Foundation. In the mean-time she joined the ITEP as a part-time laboratory assistant and the NRNU MEPhI as a part-time engineer. In August 2016, Mariia performed as a tutor for Astrophysical school (by the Non-profit Initiatives Traektoria Foundation) in the Special Astrophysical Observatory RAS.

In 2016, Mariia started as a Ph.D. candidate under the supervision of Prof. Dr. Cristiane de Morais Smith at the Institute for Theoretical Physics in the Utrecht University and at the Institute for Theoretical Physics of the University of Amsterdam under the supervision of Dr. Jasper van Wezel. This collaboration was a part of the Delta Institute for Theoretical Physics Consortium, a program of the Netherlands Organization for Scientific Research. The results of her research performed in the four years of this doctoral program are presented in this Thesis.

During her bachelor's, master's, and Ph.D. studies Mariia was a teaching assistant for several courses: Functional Series, Theoretical Mechanics, Quantum Matter, Student seminar, and Quantum Mechanics. She performed as a co-supervisor for four bachelor projects together with Dr. Jasper van Wezel. She presented her research in Russia, The Netherlands, France, Finland, Germany, and The United Kingdom in more than forty-five seminars, conferences, and schools. Mariia earned 
a top-three best poster nomination with her work "Mobility edge wave functions in charge-density waves" at Physics@Veldhoven (in 2018). Furthermore, Mariia was a referee for the following scientific journals, namely International Journal of Theoretical Physics, The European Physical Journal B, and Nonlinearity. Mariia was an organizer of International School on Ultra-Intense Lasers (in 2015) and The Dutch Research School of Theoretical Physics (DRSTP) SPTCM school (in 2018). For the latter she became the invited member of the Educational Board in DRSTP (in 2018).

In her spare time, Mariia is interested in popularization of science. She was an organizer of the open days of the Faculty of Experimental and Theoretical Physics in the NRNU MEPhI (from 2014 to 2016). Since 2017 she has a pop-science Instagram blog (@science.therapy). Mariia was an invited lecturer in Astrophysical school organized by the Non-profit Initiatives Traektoria Foundation with several lectures in Pushchino, Russia, and Turku, Finland in April and August 2018. In April 2020 she was an invited speaker for an on-line colloquium with lecture "Everything you always wanted to know about academic career but were afraid to ask" for the Student Science Society of the NRNU MEPhI. Moreover, in August 2020 Mariia was an invited speaker with lecture "The recipe for a good poster" for Tournament of Three Sciences organized by the NRNU MEPhI. 


\section{Acknowledgement}

Together with finishing my postgraduate studies, I complete my (first) decade in physics. A lot of people and events influenced my life during this period. I am grateful to everyone because of whom and despite whom I have written this Thesis, because otherwise, I would not have defended this Thesis on September 16, 2020, but it was definitely worth it.

In general, I want to say thank you to all people with whom I met in the NRNU MEPhI (especially the Department for Theoretical Nuclear Physics and Sergey V. Popruzhenko), the NRC Kurchatov Institute ITEP (especially the Elementary Particle Theory Laboratory), the Utrecht University and the University of Amsterdam (especially the groups of Jasper van Wezel and Jean-Sébastien Caux).

Now, I want to dwell on some special people that have a direct impact on this Thesis. I try to do it in chronological order.

First, dear Nikolay Gnezdilov and Andrew Pavlov, without you this all would be impossible at all. Thank you both for your kind support in searching for a Ph.D. position in 2015 .

Dear Cristiane de Morais Smith, first of all, thank you for answering on my mail in February, 2016 and for this great opportunity to work with you. I realize that during our four years we did not work together that much. However, it is not the quantity but the quality of interactions that matter. I think there were enough of them for me to adopt the best from you, and I hope you are proud of me now.

Dear Jasper van Wezel, I am grateful that you believed in my ideas, trusted in my work-style, and for your absence of any fears concerning my creativity. I am very happy that I can "give you as a gift" the JHEP paper (let's see, what will happen with it, but at least we tried).

Dear Oleksandr Gamayun, thank you for your patience in April and May, 2020. The work that we completed during this period is one of the best in my career. I was amazed at how easy-going, qualified, and smart you are. I hope you find the job of your dreams in the coming months and that you work with people who deserve you. 


\section{Acknowledgement}

Then, dear Olga Solovjeva, thank you that in November 2018 you invited me to your home in Frankfurt am Main and, by accident, showed me the paper of Alexander Molochkov, in which the modeling of the structure of protein backbone with the help of kinks is discussed. The inquisitive nature of your mind opened new horizons for my research.

Dear Irina Bode and Ann Mukhortava, I am still extremely happy that you have helped me with learning more about the proteins and participated in selecting relevant references for the introduction.

Finally, dear Alexander Barnaveli, you made a really great photo of me for the Thesis, exactly as I wanted.

Then, I would like to focus on some colleagues who became special for me during the period of 2016 - 2020 .

Dear Lars Fritz, I want to note your professionalism, tact, openness, and honesty. The combinations of these characteristics allowed me to enjoy twice the teaching experience with you, overcome the fear of asking a question, and become more open to the world in general. I think the main result of our communication is that you forced me to order my first glasses, and I finally found out how beautiful is the world around me. Thank you a lot.

Dear Non-profit Initiatives Traektoria Foundation, we both know that we are united by more than just a friendship. It is very hard to highlight someone from your big family, so I will avoid doing it here. I am just very happy that there is an office in Moscow, where I can always come and I will be welcome. This is a nice island of stability in our fast-changing world.

Dear Student Science Society of the NRNU MEPhI (especially Agnia Borodina), you made my dream come true, when you invited me to be a lector on on-line colloquium in April, 2020. You work very professionally, and I am looking forward to continuing our collaboration.

Particular acknowledgment to Natalia and Vass Verkhodanova, who made my moving from Russia to The Netherlands smoother by providing me a room for a first week in Eindhoven and moral support on time.

Last but not the least, I want to acknowledge my friends, who have been an important part of my life in the last four years: Alexander Barnaveli, Maria Bogdanova and your son Lesha, Anna Bondarevskaya, Diana Grishina and Alexander Kryuchkov, my dear Ilia Grishmanovskii, Anna Korsakova, Anastasia Maksimicheva and your children Lesha and Yasya, Ekaterina Masgutova, Andrew and Victoria Mende, Daria Rumyantseva, Olga Ryabukhina, Marina Shturmina, Yury Smirnov, Olga Solovjeva and Vasily Tulsky, Maria Tikhanovskaya, Jekaterina Titova, and Georgy Volkov. I appreciate each of you, with each of you I have special stories, to each of you I am grateful for different reasons. Just, please, continue to stay in my life. 
In the end, dear Jan Mulder and Heleen Schuttevaêr. Heleen, you are the best landlady in the world, I am sure. Thank you for not complaining about my night work, especially during the quarantine, when I was in my active phase of writing the Thesis. I think we have spent good weeks together during the pandemic. Not everyone can go through months of quarantine together with no quarrels. We have managed. I think we are ready to go through the next wave of the pandemic, together with my beloved Mark, naturally. 


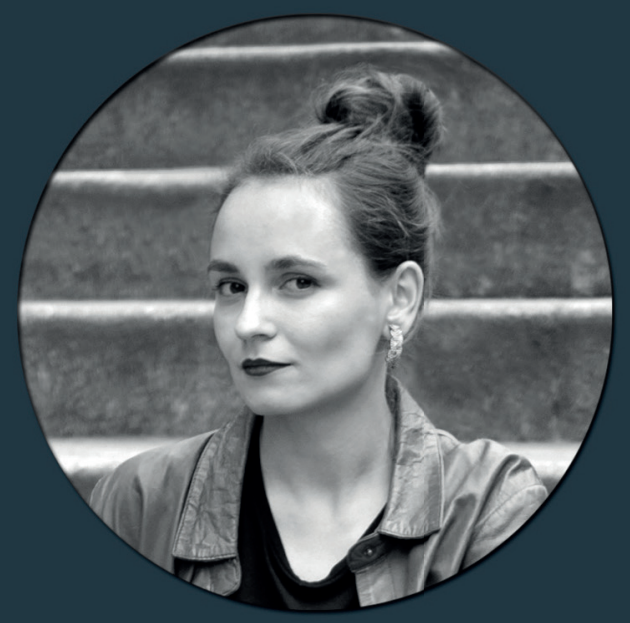

Mariia Andreyevna Lizunova (also known as Mariya Lizunova) is a theoretical physicist.

She finished her master cum laude in the NRNU MEPhI (Russia) in 2016. In 2016-2020 Mariia was a Ph.D. student in the UU and the UvA (The Netherlands).

In her spare time, Mariia is interested in the popularization of science. She was an organizer of the faculty open days in the NRNU MEPhI. Since 2017 she has a pop-science Instagram blog (@science.therapy).

Mariia was invited as a speaker with several lectures for the Non-profit Initiatives Traektoria Foundation and the Student Science Society of the NRNU MEPhI between 2018 and 2020.

This Thesis is the result of four years of work, done at the UU and the UvA under the supervision of Prof. Dr. Cristiane de Morais Smith and Dr. Jasper van Wezel. 